Obstrução de Euler de Aplicações Analíticas

Nivaldo de Góes Grulha Júnior 



\title{
Obstrução de Euler de Aplicações Analíticas
}

\author{
Nivaldo de Góes Grulha Júnior
}

Orientadora (Brasil): Profa. Dra. Maria Aparecida Soares Ruas

\author{
Orientador (França): Prof. Dr. Jean-Paul Brasselet
}

Tese em Co-tutela apresentada ao Instituto de Ciências Matemáticas e de Computação - ICMC-USP e ao Institut de Mathématiques de Luminy Université de la Méditerranée, como parte dos requisitos para obtenção do título de Doutor em Ciências Matemática.

\section{USP - São Carlos \\ Outubro/2007}



À minha avó Elza e aos meus avós (In memoriam) Artur, Âurea e Nestor. 

Aos meus pais, Nivaldo e Nilce e à minha esposa Suelen. 

Agradeço primeiramente a Deus, a Ele toda honra e toda glória. Obrigado Senhor, por ter me sustentado por todo esse caminho, me dando força e ânimo.

Aos meus pais por todo apoio que me deram, tanto financeiro como moral, pelo incentivo e, principalmente, por acreditarem em mim. Pelos puxões de orelha quando, mais novo, preferia jogar bola à estudar. Obrigado, esse doutorado também é de vocês. Aos meus irmãos, Thiago, Thales e Ígor, valeu manos. À minha esposa Suelen que esteve ao meu lado nestes últimos anos de doutorado, por ter me apoiado com muito amor, paciência e companheirismo, pelas alegrias somadas e problemas compartilhados, obrigado por estar na minha vida. À minha querida avó Elza, pelas orações. Aos meus sogros Israel e Lúcia, obrigado por tudo. À toda minha família que sempre torceu por mim, muito obrigado, amo todos vocês.

À todos os professores que tive até aqui, queridos mestres da E. M. Armando C. Riguetti, E. E. Dom Pedro I e, principalmente, ao professores do ICMC/USP, agradeço a Deus pela oportunidade de ter estudado aqui, tendo contato com matemáticos de tão alto nível.

Entre os professores do ICMC gostaria de fazer um agradecimento especial aos professores Wagner e Sandra, vocês sempre acreditaram em mim e foram importantíssimos para que eu não desistisse do sonho que estou realizando agora. Aos professores Marcelo e Daniel, que por várias vezes se dispuseram do seu tempo para tirar minhas dúvidas bobas com toda humildade e consideração, e por sempre acreditarem no meu trabalho, muito obrigado.

Tive o privilegio de ser orientado por dois grandes matemáticos, Profa. Cidinha e Prof. Brasselet. Foi uma honra ter trabalhado com esses grandes nomes da matemática. Muito obrigado pela paciência em responder várias vezes as mesmas perguntas, pelo apoio nos momento em que eu achava que não conseguiria, pela disposição para auxiliar-me na pesquisa, pela amizade, paciência, confiança, compreensão, sugestões e leitura de cada linha deste trabalho. Além de aprender matemática, aprendi com vocês muito sobre humildade, trabalho e respeito. Vocês foram realmente muito mais do que orientadores, obrigado.

Quero ainda fazer um agradecimento especial ao Prof. Brasselet e a Simone pelo apoio que minha esposa e eu recebemos enquanto estivemos na França, pela amizade, carinho, atenção e é claro, pelos jantares, passeios e churrascos maravilhosos, obrigado.

Aos meu amigo Thiago, sem ele essa tese teria sido datilografada e os desenhos feitos a mão, e Everaldo pelo apoio como amigo e como matemático. Aos meus amigos da USP, que de uma forma ou de outra me ajudaram nessa caminhada (Aldício, Ana Carla, Andréa, Angela, Anselmo, Aurélio, Benito, Cristiane, Daniela, Esdras, Fernando, Grazielle, João, José Paulo, Judith, Juliano, Junior (Xaropinho), Lizandro, Marcela, Marcos, Marcus, Mário, Mário (Ronaldinho), Meryelen, Michelle Pierri, Michelle, Miriam, Rodolfo, Rodrigo (Urso), Rodrigo Rodrigues, Rogério (Bléia), Tatiana).

Aos funcionários do ICMC/USP e funcionários do IML/Université de La Méditerranée, muito obrigado.

Aos irmãos da Assembléia de Deus Bom Retiro de São Carlos e da Primeira Igreja Batista em São Miguel Paulista, muito obrigado pela amizade e carinho.

À FAPESP e à CAPES pelo apoio financeiro para realização deste trabalho, muito obrigado.

"Então Samuel tomou uma pedra, e a pôs entre Mizpá e Sem, e lhe chamou Ebenézer; e disse: Até aqui nos ajudou o Senhor". 



\section{Resumo}

Neste trabalho determinamos relações entre a obstrução de Euler de uma função analítica com singularidade isolada $f$ e o número de Milnor de $f$ definido por Bruce e Roberts para funções definidas em espaços singulares. Apresentamos também uma generalização da obstrução de Euler de uma função analítica com singularidade isolada para o caso de uma aplicação analítica $f$ : $(V, 0) \rightarrow\left(\mathbb{C}^{k}, 0\right)$, onde $(V, 0)$ é o germe de uma variedade analítica complexa, equidimensional de dimensão $n \geq k$, e uma fórmula para calcular a obstrução de Euler de $k$-referenciais, em termos da obstrução de Euler de $f$. 



\section{Résumé}

Dans ce travail on détermine des relations entre l'obstruction d'Euler local d'une fonction analytique singulière à l'origine $f$ et du nombre de Milnor de $f$ défini par Bruce et Roberts pour des fonctions sur les espaces singuliers. On present aussi une généralisation pour l'obstruction d'Euler locale d'une fonction analytique singulière à l'origine dans le cas d'une application holomorphe $f:(V, 0) \rightarrow$ $\left(\mathbb{C}^{k}, 0\right)$, où $(V, 0)$ est un germe de variété analytique complexe, équidimensionnel de dimension $n \geq k$, et une formule qu' exprime l'obstruction d'Euler locale, définie pour un $k$-repère, en fonction de l'obstruction d'Euler relative à $f$. 



\begin{abstract}
In this work we determine relations between the local Euler obstruction of an analytic function singular at the origin $f$ and the Milnor number of $f$ defined by Bruce and Roberts for functions on singular spaces. We also present a generalization of the local Euler obstruction of a analytic function singular at the origin to the case of a analytic map $f:(V, 0) \rightarrow\left(\mathbb{C}^{k}, 0\right)$, where $(V, 0)$ is the germ of a complex analytic variety, equidimensional of dimension $n \geq k$, and a formula which computes the local Euler obstruction, defined for $k$-frames, in terms of the local Euler obstruction of $f$.
\end{abstract}



1 Requisitos Básicos $\quad 9$

1.1 Fibrados Vetoriais . . . . . . . . . . . . . . . . . . . . 9

1.2 Campos de Vetores . . . . . . . . . . . . . . . . . . . . . . . 11

1.3 Teoria de Singularidades . . . . . . . . . . . . . . . . . . . 11

1.4 Variedades Algébricas . . . . . . . . . . . . . . . . . . . . . . 14

1.5 Espaços Analíticos Complexos . . . . . . . . . . . . . . . . . . . 15

1.6 Estratificação de Whitney . . . . . . . . . . . . . . . . . . . 16

1.7 Modificação de Nash . . . . . . . . . . . . . . . . . . . . . . . . . 18

1.8 Variedades Polares . . . . . . . . . . . . . . . . . . . . . . . . . . . 19

1.9 Multiplicidades Polares . . . . . . . . . . . . . . . . . . . . . 25

2 Obstrução de Euler Local $\quad 27$

2.1 Obstrução de Euler Local . . . . . . . . . . . . . . . . . . . . . . 27

2.2 Fórmula de Gonzalez-Sprinberg e Verdier . . . . . . . . . . . . . . . 28

2.3 Obstrução de Euler Local e Multiplicidades Polares . . . . . . . . . . . . . . . . . . 31

2.4 Uma Fórmula do Tipo Lefschetz . . . . . . . . . . . . . . . . . . . . . . . . 33

3 Obstrução de Euler de $f \quad 37$

3.1 Singularidades Isoladas de Funções . . . . . . . . . . . . . . . . . . . . 38

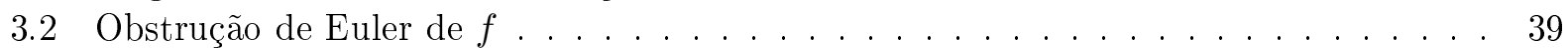

3.3 Morsificação de funções . . . . . . . . . . . . . . . . . . . . . . 42

3.4 O Link Complexo . . . . . . . . . . . . . . . . . . . . . . . . . . . . 43

3.5 Profundidade Homotópica Retificada . . . . . . . . . . . . . . . . . . . . 44

3.6 Generalizações do Número de Milnor . . . . . . . . . . . . . . . . . . . . . . 46

3.7 Número de Milnor de Bruce e Roberts . . . . . . . . . . . . . . . . . . . . . . . 48

4 Obstrução de Euler de uma Aplicação $\quad \mathbf{5 7}$

4.1 Obstrução de Euler de um $k$-campo . . . . . . . . . . . . . . . . . . 57

4.2 Caso de um $k$-campo obtido por prolongamento radial. . . . . . . . . . . . . 58

4.3 Caso de um $k$-campo qualquer. . . . . . . . . . . . . . . . . . . . 59

4.4 Obstrução de Euler de uma Aplicação Analítica . . . . . . . . . . . . . . . . . . . 60

4.5 Teorema Principal . . . . . . . . . . . . . . . . . . . . . . . 62

4.6 Caso de Variedades Regulares . . . . . . . . . . . . . . . . . . . . . . . . 65

4.7 Caso Equidimensional . . . . . . . . . . . . . . . . . . . . . . 66

4.8 Comentários Finais . . . . . . . . . . . . . . . . . . . . . . . 68

$\begin{array}{ll}\text { Referências Bibliográficas } & 71\end{array}$ 
O conceito de classes características para variedades diferenciáveis foi introduzido em 1935 independentemente, por Stiefel, e por H. Whitney. Em seu artigo [14] de 1945, Chern deu várias definições equivalentes para classes características sobre variedades hermitianas, a partir de então chamadas classes de Chern. Uma referência para o estudo de classes características de variedades regulares é o livro de J. Milnor e J. Stasheff (ref. [53]).

O primeiro exemplo de classe característica é a característica de Euler-Poincaré definida para poliedros finitos de dimensão dois por Euler e nas demais dimensões por Poincaré, como a soma alternada sobre $i$ do número de simplexos de dimensão $i$. A fórmula de Poincaré-Hopf fornece uma interpretação geométrica da característica de Euler-Poincaré de uma variedade compacta suave $M$, em termos da obstrução à construção de um campo de vetores tangentes à $M$. Mais tarde, foram descobertas outras interpretações da característica de Euler-Poincaré, mostrando a importância desta noção. Em [47], E. L. Lima dá uma excelente visão geral sobre este invariante, que tem se revelado um verdadeiro traço de união entre diferentes ramos da Matemática, aparecendo como resultado de contagens ou avaliações em Topologia, Equações Diferenciais, Geometria Diferencial e Análise.

As classes de Chern, no caso complexo e as de Stiefel-Whitney, no caso real generalizam a noção da característica de Euler-Poincaré. Elas foram primeiramente definidas como obstrução à construção de campos de $k$-referenciais tangentes a uma variedade suave, tanto no caso complexo quanto no caso real.

A extensão do conceito de classes características para variedades singulares depende de uma generalização adequada para o conceito de fibrado tangente. Existem pelo menos três maneiras de obter essa generalização. A primeira consiste em considerar a união dos fibrados tangentes aos estratos de uma estratificação da variedade, e de considerar a obstrução à construção de $k$ referenciais adaptados. Este é o ponto de vista de M.-H. Schwartz, que em [59] introduz o método de construção de campos radiais para apresentar a primeira definição de classes características para variedades singulares analíticas complexas [58]. A definição de R. MacPherson [49], utilizando o fibrado de Nash, generaliza a definição axiomática das classes de Chern, com uma prova da conjectura de Deligne e Grothendieck sobre a existência e unicidade de classes características de variedades singulares. J.-P. Brasselet e M.-H.Schwartz mostram em [10], via o isomorfismo de Alexander, que as definições de M.-H. Schwartz e de R. MacPherson são as mesmas, e desde então estas classes têm sido chamadas de classes de Schwartz-MacPherson. Uma referência básica para 
este assunto é o trabalho de J.-P. Brasselet [4].

Outra maneira de substituir o fibrado tangente, no caso de uma variedade singular, é considerar o fibrado virtual. Este é o ponto de vista de Fulton em sua definição de classes características de variedades singulares [19], as quais são em geral distintas das classes de Schwartz-MacPherson. Esta diferença tem sido investigada por vários autores e é chamada classe de Milnor. Para interseção completa com singularidade isolada, ela é obtida como a soma dos números de Milnor nos pontos singulares $[8,66]$.

A obstrução local de Euler de uma variedade algébrica $V$ em um ponto $p$, denotada por $E u_{V}(p)$, definida por MacPherson, foi um dos principais ingredientes da sua prova da conjectura de Deligne e Grothendieck [49]. Uma outra definição foi dada em [10] por J.-P Brasselet e M.-H. Schwartz, que obtêm, nesse mesmo artigo, a equivalência entre as duas definições.

Este invariante não é facilmente computável a partir de sua definição, o que motivou a obtenção de fórmulas que facilitassem o seu cálculo. Gonzalez-Sprinberg e Verdier obtiveram uma fórmula para a obstrução de Euler em função das classes de Chern do fibrado de Nash associado à variedade $V$ [24], que por sua vez, foi utilizada por Lê Dũng Tráng e Bernard Teissier para obter uma fórmula em função das multiplicidades polares de $V$ [45]. V. H. Jorge-Perez, D. Levcovitz e M. J. Saia em [38], utilizando o trabalho de Lê D. T. e Bernard Teissier, mostraram a importância da obstrução de Euler no contexto de aplicações.

J.-P. Brasselet, Lê D. T. e J. Seade obtiveram em [7], uma fórmula do tipo Lefschetz, portanto mais topológica para a obstrução de Euler, a partir da qual se obtém que a obstrução de Euler local, como função construtível, satisfaz à condição de Euler com relação às formas lineares genéricas. Uma seqüência natural desse resultado foi o artigo de J.-P. Brasselet, D. Massey, A. J. Parameswaran e J. Seade [9], cujo objetivo é entender o que impede a obstrução de Euler local de satisfazer à condição de Euler para funções analíticas com singularidade isolada na origem. Esta obstrução é chamada de obstrução de Euler local de $f$.

Um importante invariante de um germe de função analítica $f:\left(\mathbb{C}^{n}, 0\right) \rightarrow(\mathbb{C}, 0)$ com ponto crítico isolado na origem é o seu número de Milnor, denotado por $\mu(f)$, definido como sendo $\operatorname{dim}_{\mathbb{C}} \mathcal{O}_{n} / J(f)$, onde $\mathcal{O}_{n}$ é o anel dos germes na origem de funções analíticas e $J(f)$ é o ideal jacobiano de $f$.

Este invariante fornece várias informações sobre a geometria de $f$, como por exemplo, no caso em que $f$ tem ponto crítico isolado na origem, os seguintes invariantes coincidem a menos de sinal.

(a) O número de Milnor de $f$ em 0 , denotado por $\mu(f)$;

(b) O número de pontos de Morse de uma Morsificação da $f$;

(c) O índice de Poincaré-Hopf do campo gradiente de $f$ conjugado $\overline{\nabla f}$.

Quando $(V, 0)$ é o germe de um espaço analítico complexo mergulhado em $\mathbb{C}^{n}$, uma das possíveis generalizações de $(c)$ é a obstrução de Euler de $f$ na origem.

Em [60] J. Seade, M. Tibar e A. Verjovsky mostram que a obstrução de Euler de $f$ está intimamente ligada ao número de pontos de Morse de uma Morsificação de $f$ para algumas categorias de germes de variedades singulares. Eles também comparam $E u_{f, V}(0)$ com duas diferentes generalizações do número de Milnor para funções com singularidade isolada sobre espaços singulares, a noção de número de Milnor devida a Lê D. T. [40] e outra definida por David Mond, D. van 
Straten [54] e V. Goryunov [25] para curvas e também considerada por T. Izawa e T. Suwa [37] para funções definidas em interseções completas em geral. Quando $(V, 0)$ é uma interseção completa com singularidade isolada, os autores em [60] também estudaram o índice GSV definido em [23].

Neste trabalho damos continuidade a este estudo, introduzindo um novo conceito de obstrução de Euler para aplicações definidas em variedades singulares a valores em espaços de dimensão complexa maior do que um. Também descrevemos fórmulas relacionando a obstrução de Euler e outros invariantes da teoria de singularidades e apresentamos algumas aplicações dos resultados.

Os resultados originais deste trabalho se iniciam através do estudo da noção de número de Milnor introduzida por J. W. Bruce e M. Roberts para funções definidas sobre uma variedade algébrica singular em [13], aqui denotado por $\mu_{B R}(f)$.

Apresentamos relações entre $E u_{V, f}(0)$ e $\mu_{B R}(f)$, para $f$ com singularidade isolada definida em uma variedade analítica $V$, tal que a sua variedade logarítmica característica associada, denotada por $L C(V)$, seja Cohen-Macaulay. No Teorema 3.61 obtemos a seguinte fórmula,

$$
\mu_{B R}(f)=\sum_{\alpha=0}^{d} m_{\alpha}(-1)^{\operatorname{dim}_{\mathbb{C}} V_{\alpha}} E u_{f, \bar{V}_{\alpha}}(0),
$$

onde as multiplicidades $m_{\alpha}$ dependem dos estratos $\left\{V_{\alpha}\right\}$ de uma estratificação de Whitney específica de $V$ [29]. Como aplicação, mostramos que o número de Milnor de Bruce e Roberts é um invariante topológico para famílias de funções com singularidade isolada definidas em hipersuperfícies com singularidade isolada $V$. Neste caso sabemos que $L C(V)$ é uma variedade Cohen-Macaulay (Teorema $3.60)$.

Um passo natural para a generalização da obstrução de Euler de uma função é a obstrução de Euler de uma aplicação $f:(V, 0) \rightarrow\left(\mathbb{C}^{k}, 0\right)$, onde $(V, 0)$ é um germe de variedade analítica complexa, equidimensional de dimensão $n \geq k$. Analogamente ao caso de funções, definimos a obstrução de Euler de uma aplicação analítica $f$, em função de um $k$-referencial que depende das funções coordenadas da aplicação $f$.

A obstrução de Euler local associada a um $k$-referencial sobre uma variedade analítica foi estudada por J.-P. Brasselet, J. Seade e T. Suwa em [11]. Um dos principais resultados aqui apresentados é uma fórmula que relaciona a obstrução de Euler local associada a um $k$-referencial sobre uma variedade analítica e a obstrução de Euler de uma aplicação analítica $f:(V, 0) \rightarrow\left(\mathbb{C}^{k}, 0\right)$, onde $(V, 0)$ é um germe de variedade analítica complexa, equidimensional de dimensão $n \geq k$ [30].

Esta tese contém quatro capítulos, que estão divididos em três grupos, requisitos básicos, resultados anteriores e resultados originais do trabalho, conforme descrevemos a seguir.

No primeiro capítulo apresentamos alguns requisitos básicos para o desenvolvimento do trabalho, como os conceitos de fibrados vetoriais, teoria de singularidades, variedades algébricas e analíticas, campos vetoriais, teoria de estratificação e modificação de Nash. Introduzimos também o conceito de variedades polares, dando exemplos básicos para uma melhor compreensão do conceito.

No segundo capítulo definimos a obstrução de Euler local e apresentamos os principais resultados envolvendo este conceito, como o teorema de Gonzalez-Sprinberg e Verdier, o Teorema de Lê-Teissier e a fórmula do tipo Lefschetz de J.-P. Brasselet, Lê D. T. e J. Seade.

Introduzimos no terceiro capítulo a obstrução de Euler de uma função, e apresentamos os principais resultados envolvendo a comparação da obstrução de Euler de $f$ com generalizações do número 
de Milnor, incluindo um resultado original comparando a obstrução de Euler de $f$ com o número de Milnor de Bruce e Roberts.

No quarto capítulo definimos e estudamos a obstrução de Euler de uma aplicação analítica e apresentamos uma fórmula que generaliza para aplicações definidas em variedades analíticas, os resultados de J.-P. Brasselet, D. Massey, A. J. Parameswaran e J. Seade para funções. 


\section{Introduction}

Le concept de classes caractéristiques a été introduit en 1935 indépendamment, par Stiefel et par Whitney. Dans son article [14] de 1945, Chern a donné plusieurs définitions équivalentes pour les classes caractéristiques des variétés hermitiennes, on les a alors apelées classes de Chern. Une bonne référence pour l'étude des classes caractéristiques des variétés lisses est [53].

La caractéristique d'Euler-Poincaré, définie pour un polyèdre fini comme la somme alternée sur $i$, des nombres de simplexes de dimension $i$, est le premier exemple de classe caractéristique. Cette definition a été donnée par Euler en dimension deux et par Poincaré pour les autres dimensions. La formule de Poincaré-Hopf nous donne une interprétation géométrique de la caractéristique d'EulerPoincaré, dans les cas d'une variété lisse compacte $M$, en termes d'obstruction à la construction d'un champ de vecteurs tangent à $M$.

Par la suite, d'autres interprétations de la caractéristique d'Euler- Poincaré on permis de montrer l'importance de cette notion. L'article [47] donne une excellente vision générale sur cet invariant, qui s'est révélé un réel trait d'union entre différentes branches des mathématiques, en particulier Topologie, Équations Différentielles, Géométrie Différentielle et Analyse.

Les classes de Chern, dans le cas complexe et les classes de Stiefel-Whitney, dans le cas réel, généralisent la notion de caractéristique d'Euler-Poincaré. Ces classes ont été tout d'abord définies comme obstruction à la construction de champs de $k$-repères tangents à une variété lisse, tant dans le cas complexe que dans le cas réel.

L'extension du concept de classe caractéristique au cas des variétés singulières dépend d'une généralisation appropriée du concept de fibré tangent. Il exists au moins deux manières d'obtenir cette généralisation. La première consiste à considérer l'union des fibrés tangents au strates d'une stratification de la variété, et de considérer l'obstruction à la construction de $k$-repères adaptés. Ceci est le point de vue de M.-H. Schwartz, qui en [59] introduit la méthode de construction des champs radiaux pour présenter la première définition de classes caractéristiques pour les variétés singulières analytiques complexes [58]. La définition de R. MacPherson [49] généralise la définition axiomatique des classes de Chern et fournit une preuve de la conjecture de Deligne et Grothendieck. Une référence basique pour ce sujet est [4].

J.-P. Brasselet et M.-H. Schwartz ont montré en [10] que les définitions de M.-H. Schwartz et de R. MacPherson correspondent par isomorphisme de Alexander, et depuis lors elles ont été appelées classes de Schwartz-MacPherson.

Une autre manière de remplacer le fibré tangent, dans le cas d'une variété singulière, est de 
considérer le fibré virtuel. Ceci est le point de vue de Fulton dans sa définition de classes caractéristiques de variétés singulières [19], lesquelles sont, en général, différentes des classes de SchwartzMacPherson. Ces classes ont été étudiées en [1] par P. Allufi dans le cas des hypersuperfaces.

Pour une hypersurface et plus généralement, pour une intersection complète locale $V$, il est possible d'interpréter la différence entre classes de Schwartz-MacPherson et classes de Fulton. Si $V$ a des points singuliers isolés, la différence est la somme des nombres de Milnor en les points singuliers $[8,66]$. Dans le cas général plusieurs auteurs donnent des interprétations pour cette différence, qui est appelée classe de Milnor.

Si $p$ est un point d'une variété algébrique $V$, l'obstruction d'Euler locale $E u_{V}(p)$, définie par MacPherson, est l'un des principaux ingrédients de sa preuve de la conjecture de Deligne et Grothendieck [49]. Une autre définition pour l'obstruction d'Euler a été donnée par J.-P. Brasselet et M.-H. Schwartz en [10], où l'équivalence entre les deux définitions a aussi été obtenue.

L'obstruction d'Euler n'était pas facile à calculer à partir de sa définition, des formules ont été développées pour en faciliter le calcul. Ainsi Gonzalez-Sprinberg et Verdier ont montré une formule pour l'obstruction d'Euler en fonction des classes de Chern du fibré de Nash associé à la variété $V$ [24]. En utilisant cette formule, Lê Dũng Tráng et Bernard Teissier ont prouvé une formule en fonction des multiplicités polaires de $V$ [45].

V. H. Jorge-Perez , D. Levcovitz et M. J. Saia en [38], en utilisant le travail de Lê D. T. et Bernard Teissier, ont montré l'importance de l'obstruction d'Euler dans le contexte des applications.

D'autre part, dans [7] J.-P. Brasselet, Lê D. T. et J. Seade donnent une formule du type de Lefschetz donc plus topologique, pour l'obstruction d'Euler locale, alors que le résultat de [45] est de nature algébrique. La formule prouvée revient à dire que l'obstruction d'Euler locale, comme fonction constructible, satisfait la condition d'Euler en ce qui concerne les formes linéaires génériques.

Une suite naturelle de ce résultat est l'article [9] dû à J.-P. Brasselet, D. Massey, A. J. Parameswaran et J. Seade, où le but est de comprendre ce qui empêche l'obstruction d'Euler locale de satisfaire la condition d'Euler en ce qui concerne les fonctions holomorphes $f:(V, 0) \rightarrow(\mathbb{C}, 0)$ singulières à l'origine. Le défaut est appelé obstruction d'Euler locale de $f$.

Un important invariant d'un germe d'une fonction analytique ayant un point critique isolé à l'origine $f:\left(\mathbb{C}^{n}, 0\right) \rightarrow(\mathbb{C}, 0)$ est son nombre de Milnor, noté $\mu(f)$ et défini comme $\operatorname{dim}_{\mathbb{C}} \mathcal{O}_{n} / J(f)$, où $\mathcal{O}_{n}$ est l'anneau des germes à l'origine de fonctions analytiques et $J(f)$ est l'ideal jacobien de $f$.

Cet invariant fournit beaucoup d'informations sur la géométrie de $f$. Par exemple, quand $f$ a un point critique isolé à l'origine, les invariants suivants coïncident au signe près.

(a) Le nombre de Milnor de $f$ à l'origine;

(b) Le nombre de points de Morse d'une morsification de $f$;

(c) L'indice de Poincaré-Hopf du champ gradient conjugué de $f$ (noté $\overline{\nabla f}$ ).

Supposons maintenant que $(V, 0)$ est le germe d'un espace analytique complexe plongé dans $\mathbb{C}^{n}$. Une des généralisations possibles de $(c)$, introduite dans [9] est l'obstruction d'Euler de $f$ à l'origine, notée $E u_{V, f}(0)$. 
En [60] J. Seade, M. Tibar et A. Verjovsky ont montré que l'obstruction d'Euler de $f$ est très liée au nombre de points de Morse d'une morsification de $f$ pour quelques types de germes de variétés singulières. Ils ont aussi comparé $E u_{f, V}(0)$ avec deux généralisations différentes du nombre de Milnor pour les fonctions avec singularité isolée stratifiée sur les espaces singuliers : la notion de nombre de Milnor dûe à Lê D. T. [40] et celle dûe à David Mond, D. van Straten [54] et V. Goryunov [25] pour les courbes et à T. Izawa et T. Suwa [37] pour les fonctions définies sur les intersections complètes en général. Quand $(V, 0)$ est une intersection complète avec singularité isolée les auteurs ont aussi étudié l'indice GSV [23].

Dans le présent travail on continue cette étude, en introduisant un nouveau concept d'obstruction d'Euler pour les applications définies sur des variétés singulières à valeur dans des espaces complexes de dimension plus grande que 1 . On décrit aussi des formules qui donnent des relations entre l'obstruction d'Euler et d'autres invariants de la théorie des singularités et on présente quelques applications de ces résultats.

Les résultats originaux de ce travail concernent également l'étude d'une autre généralisation du nombre de Milnor, à savoir la notion de nombre de Milnor définie par J. W. Bruce et R. M Roberts pour les fonctions définies sur une variété algebrique singulière [13], noté ici $\mu_{B R}(f)$.

On donne une relation entre $E u_{V, f}(0)$ et $\mu_{B R}(f)$, pour une fonction $f$ avec singularité isolée définie sur une variété analytique $\mathrm{V}$, pour laquelle la variété logarithmique caractéristique associée, not ée $L C(V)$, est Cohen-Macaulay. Par le Théorème 3.61 on a,

$$
\mu_{B R}(f)=\sum_{\alpha=0}^{d} m_{\alpha}(-1)^{a_{\alpha}} E u_{f, \bar{V}_{\alpha}}(0),
$$

où les ensembles $V_{\alpha}$ sont les éléments d'une stratification de Whitney de $V$, et les multiplicités $m_{\alpha}$ dépendent des strates de cette stratification [29]. On montre aussi que le nombre de Milnor de Bruce et Roberts est un invariant topologique pour les families de fonctions avec singularité isolée sur des hypersurfaces $V$ avec singularités isolées. Dans ce cas la variété $L C(V)$ est Cohen-Macaulay (Théorème 3.60).

Une généralization naturelle pour l'obstruction d'Euler d'une fonction est l'obstruction d'Euler d'une application $f:(V, 0) \rightarrow\left(\mathbb{C}^{k}, 0\right)$, où $(V, 0)$ est un germe de variété analytique complexe, equidimensionel de dimension $n \geq k$. Comme dans le cas des fonctions, la définition de l'obstruction d'Euler d'une application est donnée en fonction d'un $k$-repère qui dépend de l'application $f$.

L'obstruction d'Euler d'un $k$-repère sur une variété analytique a été etudiée par J.-P. Brasselet, J. Seade et T. Suwa dans [11]. L'un des résultats principaux est une formule qui exprime l'obstruction d'Euler locale, définie pour un $k$-repère en fonction de l'obstruction d'Euler relative d'une application analytique $f:(V, 0) \rightarrow\left(\mathbb{C}^{k}, 0\right)$, où $(V, 0)$ est un germe de variété analytique complexe, équidimensionnel de dimension $n \geq k[30]$.

Cette thèse contient quatre chapitres, lesquels sont divisés en trois groupes, rappels de base, résultats précédents et résultats originaux du travail, comme décrit ci-après.

$\mathrm{Au}$ premier chapitre nous rappelons quelques notions de base utiles pour le développement du travail, comme les concepts de fibré vectoriel, la théorie des singularités, variétés algébriques et analytiques, champs vectoriels, théorie des stratifications et modification de Nash. Nous introduisons aussi le concept de variétés polaires, donnant des exemples basiques pour une meilleure 
comprÈhension du concept.

Au second chapitre nous dĖfinissons l'obstruction d'Euler locale et présentons les principaux résultats concernant cette notion, comme le théorème de Gonzalez-Sprinberg et de Verdier, le théorème de Lê et Teissier et la formule du type Lefschetz de J.-P. Brasselet, Lê D. T. et J. Seade.

On introduit au troisième chapitre l'obstruction d'Euler d'une fonction, et on présente les principaux résultats sur la comparaison de l'obstruction d'Euler de $f$ avec les généralisations du nombre de Milnor, y compris un résultat original comparant l'obstruction d'Euler de $f$ avec le nombre de Milnor de Bruce et Roberts.

$\mathrm{Au}$ quatrième chapitre on définit et étudie l'obstruction d'Euler d'une application analytique et prËsentons une formule qui généralise pour les applications définies sur des variétés analytiques, les résultats de J.-P. Brasselet, D.Massey, J. Parameswaran et J. Seade pour les fonctions. 


\section{Requisitos Básicos}

Pretendemos neste capítulo dar uma breve introdução aos principais conceitos usados, como fibrados vetoriais (reais e complexos), variedades algébricas, estratificação de Whitney e transformação de Nash, procurando fornecer o mínimo necessário dessa teoria para uma boa compreensão dos resultados dos capítulos a seguir.

\subsection{Fibrados Vetoriais}

A teoria de fibrados é a uma ferramenta básica para o estudo de classes características, tanto no caso regular como no caso singular. Para um estudo mais completo sugerimos [65].

Denotemos por $B$ um espaço topológico fixado, o qual chamaremos de espaço base.

Definição 1.1: Um fibrado vetorial (real ou complexo) $\xi$ sobre $B$ é uma terna, formada por:

1. Um espaço topológico $E=E(\xi)$ chamado espaço total,

2. uma aplicação (continua) $\pi: E \rightarrow B$ sobrejetora chamada projeção,

3. para cada $b \in B, \pi^{-1}(b)$ tem uma estrutura de espaço vetorial sobre $\mathbb{K}(\mathbb{K}=\mathbb{R}$ ou $\mathbb{C}$ ).

E deve satisfazer à seguinte condição:

Condição de trivialidade local: Para cada ponto $b \in B$ deve existir uma vizinhança $U \subset B$, um inteiro $n \geq 0$ e um homeomorfismo

$$
h: U \times \mathbb{K}^{n} \rightarrow \pi^{-1}(U)
$$

tal que, para cada $b \in U$, a correspondência $x \mapsto h(b, x)$ define um isomorfismo entre o espaço vetorial $\mathbb{K}^{n}$ e o espaço vetorial $\pi^{-1}(b)$.

O par $(U, h)$ será chamado sistema de coordenadas local para $\xi$ em $b$. Se pudermos escolher $U$ igual ao espaço base, então $\xi$ será chamado de fibrado trivial. 
O espaço vetorial $\pi^{-1}(b)$ é chamado de fibra sobre $b$, e pode ser denotado por $F_{b}$ ou por $F_{b}(\xi)$. Observemos que $F_{b}$ nunca é vazio, entretanto pode ser formado por apenas um ponto.

$O$ conceito de fibrado vetorial diferenciável pode ser definido pedindo apenas que $B$ e $E$ sejam variedades diferenciáveis, que $\pi$ seja uma aplicação suave e que para cada $b \in B$ exista um sistema de coordenadas locais $(U, h)$ com $b \in U$ tal que $h$ seja um difeomorfismo.

Consideremos agora dois fibrados vetoriais $\xi$ e $\nu$ sobre o mesmo espaço base $B$.

Definição 1.2: Dizemos que $\xi$ é isomorfo à $\nu$, escrevemos $\xi \cong \nu$, se existir um homeomorfismo

$$
f: E(\xi) \rightarrow E(\nu)
$$

entre os espaços totais que aplique cada espaço vetorial $F_{b}(\xi)$ isomorficamente sobre o espaço vetorial correspondente $F_{b}(\nu)$.

Exemplo 1.3: O espaço projetivo real $\mathbb{P}^{n}$ pode ser definido como o conjunto de todos os pares $\{x,-x\}$ onde $x$ pertence à esfera unitária $\mathbb{S}^{n} \subset \mathbb{R}^{n+1}$, dotado da topologia quociente.

Seja $E\left(\gamma_{n}{ }^{1}\right)$ o subconjunto de $\mathbb{P}^{n} \times \mathbb{R}^{n+1}$ formado de todos os pares $(\{ \pm x\}, v)$, tais que $v$ seja um múltiplo de $x$. Defina $\pi: E\left(\gamma_{n}{ }^{1}\right) \rightarrow \mathbb{P}^{n}$ por $\pi(\{ \pm x\}, v)=\{ \pm x\}$. Logo cada fibra $\pi^{-1}(\{ \pm x\})$ pode ser identificada com a reta que liga os pontos $x$ e $-x$ em $\mathbb{R}^{n+1}$. Em cada reta damos uma estrutura vetorial real. O fibrado vetorial resultante $\gamma_{n}{ }^{1}$ será chamado de fibrado (canônico) de retas sobre $\mathbb{P}^{n}$.

Teorema 1.4: O fibrado $\gamma_{n}{ }^{1}$ sobre $\mathbb{P}^{n}$ é não trivial, para $n \geq 1$.

Definição 1.5: Uma seção de um fibrado vetorial $\xi$ com base $B$ é uma função contínua

$$
s: B \rightarrow E(\xi)
$$

a qual leva $b$ dentro de sua fibra correspondente $F_{b}(\xi)$. Uma seção do fibrado tangente à uma variedade suave $M$ é usualmente chamada de campo de vetores de $M$.

Se analisarmos melhor o espaço $E\left(\gamma_{n}{ }^{1}\right)$ para o caso especial $n=1$, vemos que neste caso cada ponto $e=(\{ \pm x\}, v)$ de $E\left(\gamma_{n}{ }^{1}\right)$ pode ser escrito como

$$
e=(\{ \pm(\cos \theta, \operatorname{sen} \theta)\}, t(\cos \theta, \operatorname{sen} \theta)) .
$$

Como $0 \leq \theta \leq \pi, t \in \mathbb{R}$, esta representação é única, a menos do fato que

$$
(\{ \pm \cos 0, \operatorname{sen} 0)\}, t(\cos 0, \operatorname{sen} 0)=(\{ \pm \cos \pi, \operatorname{sen} \pi)\}, t(\cos \pi, \operatorname{sen} \pi)) .
$$

Em outras palavras, $E\left(\gamma_{1}^{1}\right)$ pode ser obtido a partir da faixa $[0, \pi] \times \mathbb{R}$ no $(\theta, t)$-plano pela identificação do bordo esquerdo $\{0\} \times \mathbb{R}$ com o bordo direito $\{\pi\} \times \mathbb{R}$ pela correspondência $(0, t) \mapsto(\pi,-t)$. portanto $E\left(\gamma_{1}^{1}\right)$ é uma faixa de Möebius e logo certamente não homeomorfa ao cilindro $\mathbb{P}^{1} \times \mathbb{R}$.

Consideremos agora uma coleção $\left\{s_{1}, \ldots, s_{n}\right\}$ de seções do fibrado vetorial $\xi$.

Definição 1.6: As seções $s_{1}, \ldots, s_{n}$ são linearmente independentes se, para cada $b \in B$, os vetores $s_{1}(b), \ldots, s_{n}(b)$ forem linearmente independentes. 
Lema 1.7: Sejam $\xi$ e $\eta$ fibrados vetoriais sobre $B$ e seja $f: E(\xi) \rightarrow E(\eta)$ uma função contínua que leva cada espaço vetorial $F_{b}(\xi)$ isomorficamente sobre o espaço vetorial correspondente $F_{b}(\eta)$. Então $f$ é necessariamente um homeomorfismo. Portanto $\xi$ é isomorfo à $\eta$.

Teorema 1.8: Um fibrado vetorial $\xi$ de posto $n$ é trivial se e somente se $\xi$ admite $n$ seções linearmente independentes.

\subsection{Campos de Vetores}

Um campo de vetores $\xi$ em uma variedade diferenciável $M$ é uma correspondência que a cada ponto $p$ de $M$ associa um vetor $\xi(p) \in T_{p} M$. O campo é suave se a aplicação $\xi: M \rightarrow T M$ é suave. Considerando-se uma parametrização $x: U \subset \mathbb{C}^{n} \rightarrow M$, com $U$ aberto, é possível escrever

$$
\xi(p)=\sum_{j=1}^{n} \xi_{j}(p) \frac{\partial}{\partial x_{j}},
$$

onde $\xi_{j}: U \rightarrow \mathbb{C}, j=1, \ldots, n$ é uma função em $U$ e $\left\{\frac{\partial}{\partial x_{j}}\right\}$ é uma base associada à $x$ para $T_{p} M$. O conjunto dos campos de vetores forma um espaço vetorial sobre $\mathbb{C}$.

É conveniente pensar em um campo $\xi$ como uma aplicação $\xi: D \rightarrow D$, onde $D$ é o conjunto formado pelas funções analíticas em $M, f: M \rightarrow \mathbb{C}$, dada por

$$
(\xi f)(p)=\xi(f)(p)=\sum_{j=1}^{n} \xi_{j}(p) \frac{\partial f}{\partial x_{j}}(p),
$$

onde $f$ indica a expressão de $f$ na parametrização $x$.

\section{$1.3 \quad$ Teoria de Singularidades}

Nesta seção apresentamos as definições e principais resultados da teoria de singularidades que serão usados para o desenvolvimento deste trabalho. Estamos assumindo que o leitor já tenha algum conhecimento prévio, nos deixando livres para omitir fatos elementares. Para o leitor interessado recomendamos $[21,67,75]$.

Um dos nossos objetivos principais é estudar localmente funções analíticas $f: \mathbb{C}^{n} \rightarrow \mathbb{C}$. Para isso, introduzimos uma relação de equivalência no espaço das funções analíticas definidas num aberto contendo a origem de $\mathbb{C}^{n}$, da seguinte forma:

Definição 1.9: Dizemos que $f$ é equivalente à $g$, se existe um aberto $U \subset \mathbb{C}^{n}$ tal que $f_{\mid U} \equiv g_{\mid U}$. As classes de equivalência são chamadas de germes, denotadas por $f:\left(\mathbb{C}^{n}, 0\right) \rightarrow \mathbb{C}$, ou simplesmente, por $f$. A coleção de todos esses germes de funçôes citados acima é denotada por $\mathcal{O}_{n}$. Observemos que $\mathcal{O}_{n}$ é um anel noetheriano local cujo ideal maximal é dado por $\mathcal{M}_{n}=\left\{f \in \mathcal{O}_{n}: f(0)=0\right\}$.

Definição 1.10: Denotamos por $\mathcal{O}_{n, p}$ o conjunto dos germes de aplicações analíticas $f:\left(\mathbb{C}^{n}, 0\right) \rightarrow$ $\mathbb{C}^{p}$ e por $\mathcal{O}_{n, p}^{0}$ o conjunto dos germes $f:\left(\mathbb{C}^{n}, 0\right) \rightarrow\left(\mathbb{C}^{p}, 0\right)$.

Proposição 1.11: $\mathcal{O}_{n, p}$ é um $\mathcal{O}_{n}$-módulo livre de posto $p$. 
Uma das metas da teoria de singularidades é classificar germes de $\mathcal{O}_{n, p}$ sob relações de equivalências em $\mathcal{O}_{n, p}$ dadas por ações de grupos. Quando trabalhamos apenas com germes de funções, a relação de equivalência de nosso interesse é dada pela ação dos subgrupos do grupo dos germes de difeomorfismos de $\mathcal{O}_{n, n}$ que preservam a origem, o qual é denotado por $\mathcal{R}$. A ação é dada por $\varphi . f=f \circ \varphi^{-1}$, para todo $\varphi \in \mathcal{R}$ e $f \in \mathcal{O}_{n}$. Dizemos que dois germes $f, g \in \mathcal{O}_{n}$ são $\mathcal{R}$-equivalentes se existir um germe $\varphi \in \mathcal{R}$ tal que $f \equiv g \circ \varphi^{-1}$ assim,

$$
f \sim_{\mathcal{R}} g \Longleftrightarrow \exists \varphi \in \mathcal{R} \text { tal que } f \equiv g \circ \varphi^{-1} .
$$

O conjunto dos germes de difeomorfismos que não preservam a origem é denotado por $\mathcal{R}_{e}$ (o grupo estendido).

Dado $f \in \mathcal{O}_{n}$, para cada inteiro positivo $k$, definimos o $r$-jato de $f$ por

$$
j^{r} f=j^{r} f(0)=p_{r}(f)(0)-f(0),
$$

onde $p_{r}(f)(0)$ é o polinômio de Taylor de grau $r$ do representante $f$ no ponto $x=0$. Observemos que esse polinômio independe do representante escolhido.

Observemos que $\mathcal{O}_{n}$ não é uma variedade diferenciável de dimensão finita, e na tentativa de amenizar a dificuldade em trabalhar com espaços de dimensão infinita, trabalhamos com variedades diferenciáveis que possuem dimensão finita. Denotamos por $J^{r}(n, 1)$ o $\mathbb{C}$-espaço vetorial dos $r$ jatos dos germes de $\mathcal{O}_{n}$ e por $H^{r}$ o subespaço de $J^{r}(n, 1)$ formado pelos polinômios homogêneos de grau $r$. Portanto $J^{r}(n, 1)$ e $H^{r}$ são variedades diferenciáveis difeomorfas a algum espaço vetorial de dimensão finita. Dado $j^{r} f \in J^{r}(n, 1)$, quando não houver dúvidas denotaremos esse elemento apenas por $f$.

Da mesma forma, ao invés de trabalhar com o grupo $\mathcal{R}$, que possui dimensão infinita, trabalhamos com o conjunto $\mathcal{R}^{r}$ que denota o grupo de Lie (que age em $J^{r}(n, 1)$, veja [50]) formado pelos $r$-jatos de germes de $\mathcal{R}$.

Para a classificação de germes de funções sob uma equivalência é necessário que conheçamos o espaço tangente de uma órbita em um germe. Como o grupo $\mathcal{R}$ não é um grupo de Lie e $\mathcal{O}_{n}$ não é uma variedade diferenciável de dimensão finita, dado um inteiro positivo $r$, trabalhamos com os espaços dos $r$-jatos, $\mathcal{R}^{r}$ (que é um grupo de Lie) e $J^{r}(n, 1)$ (que é uma variedade diferenciável). Assim, podemos encontrar o espaço tangente de uma órbita em um jato. No caso do grupo $\mathcal{R}$ temos:

Dados um inteiro positivo $r$ e um germe de $r$-jato $f \in J^{r}(n, 1)$ fixados, definimos a aplicação:

$$
\begin{aligned}
\varphi_{f}: \mathcal{R}^{r} & \longrightarrow J^{r}(n, 1) \\
h & \longmapsto j^{r}\left(f \circ h^{-1}\right) .
\end{aligned}
$$

Como $\mathcal{R}^{r}$ é um aberto de $J^{r}(n, n)$ então $T_{1} \mathcal{R}^{r}=J^{r}(n, n)$. Seja $g \in T_{1} \mathcal{R}^{r}$ e consideremos a curva $\alpha:(-\varepsilon, \varepsilon) \rightarrow \mathcal{R}^{r}, \alpha(0)=1$ e $\alpha^{\prime}(0)=g$

$$
\alpha(t)(x)=x+\operatorname{tg}(x) .
$$

Então,

$$
\begin{aligned}
\frac{d}{d t}\left[\varphi_{f} \circ \alpha(t)\right]_{\mid t=0} & =\frac{d}{d t}\left[j^{r}(f \circ \alpha(t))\right]_{\mid t=0}=j^{r} \frac{d}{d t}(f \circ \alpha(t))_{\mid t=0} \\
& =j^{r} \sum_{j=1}^{n} \frac{\partial f}{\partial x_{j}}(\alpha(t)) \alpha_{j}^{\prime}(t)_{\mid t=0}=\sum_{j=1}^{n} j^{r} \frac{\partial f}{\partial x_{j}} g_{j} \in T_{f} \mathcal{R}^{r} f
\end{aligned}
$$


$\operatorname{com} g_{j} \in \mathcal{M}_{n}$ pois $\alpha(t) \in \mathcal{R}^{r}$, para qualquer $t$.

Considerando-se o ideal de $\mathcal{O}_{n}, J(f)=\left\langle\frac{\partial f}{\partial x_{j}}\right\rangle_{j=1}^{n}$, chamado de ideal jacobiano de $f$, temos $T_{f} \mathcal{R}^{r} \cdot f=j^{r}\left(\mathcal{M}_{n} J(f)\right)$, para qualquer $r$. Portanto é natural definir o espaço tangente à $\mathcal{R}$-órbita em um germe $f$ como o conjunto:

$$
T_{f} \mathcal{R} f=\mathcal{M}_{n} J(f),
$$

e o espaço tangente estendido à $\mathcal{R}$-órbita em um germe $f$ por:

$$
T_{f} \mathcal{R}_{e} f=J(f) .
$$

Para o estudo de singularidades de aplicações de $\mathbb{C}^{n} \rightarrow \mathbb{C}^{p}$, introduziremos outra noção de equivalência.

Definição 1.12: $O$ grupo $\mathcal{A}$ é o grupo dos pares de germes de difeomorfismo $(h, k)$, tais que $h$ : $\left(\mathbb{C}^{n}, 0\right) \rightarrow\left(\mathbb{C}^{n}, 0\right)$ e $k:\left(\mathbb{C}^{p}, 0\right) \rightarrow\left(\mathbb{C}^{p}, 0\right)$.

Definição 1.13: Dois germes $f_{i}:\left(\mathbb{C}^{n}, 0\right) \rightarrow\left(\mathbb{C}^{p}, 0\right), i=1,2$, são $\mathcal{A}$-equivalentes se existem germes de difeomorfismos $h:\left(\mathbb{C}^{n}, 0\right) \rightarrow\left(\mathbb{C}^{n}, 0\right)$ e $k:\left(\mathbb{C}^{p}, 0\right) \rightarrow\left(\mathbb{C}^{p}, 0\right)$ tais que, o seguinte diagrama

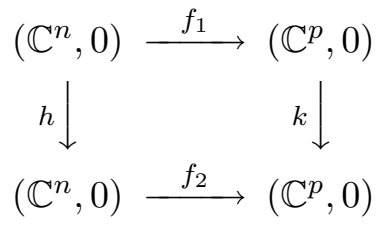

comuta.

Proposição 1.14: Consideremos o grupo $\mathcal{A}$ e o conjunto $\mathcal{O}_{n, p}^{0}$, se $f \in \mathcal{O}_{n, p}$, então a aplicação dada pela composição,

$$
(h, k) \cdot f=k \circ f \circ h^{-1} .
$$

é uma ação de grupo. Dois germes são $\mathcal{A}$-equivalentes se, e somente se, estão na mesma órbita.

Definição 1.15: $O$ grupo $\mathcal{K}$ é o grupo dos pares de germes de difeomorfismos $(h, H), H:\left(\mathbb{C}^{n} \times\right.$ $\left.\mathbb{C}^{p}, 0\right) \rightarrow\left(\mathbb{C}^{n} \times \mathbb{C}^{p}, 0\right), h:\left(\mathbb{C}^{n}, 0\right) \rightarrow\left(\mathbb{C}^{n}, 0\right)$, que comutam o seguinte diagrama

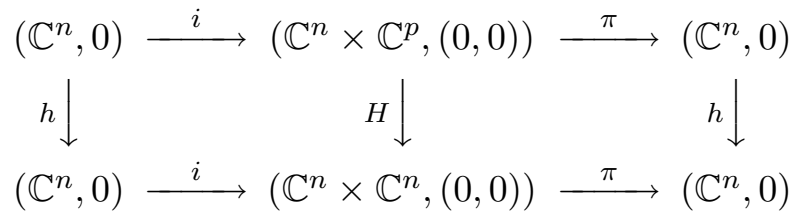

com $i$ germe de inclusão e $\pi$ germe de projeção.

Definição 1.16: Dizemos que dois germes $f_{i}:\left(\mathbb{C}^{n}, 0\right) \rightarrow\left(\mathbb{C}^{p}, 0\right), i=1,2$ são $\mathcal{K}$-equivalentes quando existe um par $(h, H)$ de germes invertíveis como acima, para os quais $H \circ\left(1, f_{1}\right)=\left(1, f_{2}\right) \circ h$ onde 1 é o germe identidade.

Proposição 1.17: Consideremos o grupo $\mathcal{K}$ e o conjunto $\mathcal{O}_{n, p}^{0}$, então a aplicação dada por $(h, H) . f=$ $H \circ(1, f) \circ h^{-1}$ é uma ação, e mais, dois germes são $\mathcal{K}$-equivalentes se, e somente se, estão na mesma órbita. 
Definição 1.18: Dado $f:\left(\mathbb{C}^{n}, 0\right) \rightarrow\left(\mathbb{C}^{p}, 0\right)$, definimos a imagem recíproca de $f$, por $f^{*}: \mathcal{O}_{p, t} \rightarrow$ $\mathcal{O}_{n, t}$ onde $f^{*}(h)=h \circ f$ e $n, p, t \in \mathbb{N}$.

Teorema 1.19: Uma condição necessária e suficiente para que $f, g:\left(\mathbb{C}^{n}, 0\right) \rightarrow\left(\mathbb{C}^{p}, 0\right)$ sejam $\mathcal{K}$ equivalentes é que exista $\phi:\left(\mathbb{C}^{n}, 0\right) \rightarrow\left(\mathbb{C}^{n}, 0\right)$ germe de difeomorfismo tal que, $\phi^{*}\left(g^{*}\left(\mathcal{M}_{p}\right) \mathcal{O}_{n}\right)=$ $f^{*}\left(\mathcal{M}_{p}\right) \mathcal{O}_{n}$, onde $\phi^{*}, g^{*}$ e $f^{*}$ são o pull back de $\phi, g$ e $f$ respectivamente, onde $\mathcal{M}_{p} \subset \mathcal{O}_{p}$ é o ideal maximal de $\mathcal{O}_{p}$.

\section{O Teorema de Fibração de Milnor}

O teorema de fibração de Milnor, descrito em [52] é um dos resultados centrais na teoria de singularidades, tendo generalizações em diversas situações.

Teorema 1.20 ([52]): Seja $f:\left(\mathbb{C}^{n}, 0\right) \rightarrow(\mathbb{C}, 0)$ germe de aplicação analítica, $V=f^{-1}(0)$, $B_{\varepsilon}$ bola centrada na origem e de raio $\varepsilon$ e $K=V \cap \mathbb{S}^{2 n-1}$. Se $\varepsilon$ for suficientemente pequeno temos que $V \cap B_{\varepsilon}$ é homeomorfo ao cone $C(K)=\{t z: 0 \leq t \leq 1, z \in K\}$.

Seja $\phi: S_{\varepsilon} \backslash K \rightarrow S^{1}$ dada por $\phi(z)=\frac{f(z)}{|f(z)|}, \operatorname{com} \phi^{-1}\left(e^{i \theta}\right)=F_{\theta}$.

O resultado básico da teoria de Milnor é o teorema da fibração, que mostra que $\phi$ define uma fibração de $S_{\varepsilon} \backslash K \rightarrow S^{1}$ com fibra $F_{\theta}$.

Teorema 1.21 ([52]): Para $\varepsilon$ suficientemente pequeno temos que $S_{\varepsilon} \backslash K$ é um fibrado diferenciável localmente trivial sobre $S^{1}$, com projeção $\phi$ e fibra $F_{\theta}$ como acima.

Podemos considerar uma fibração mais conveniente que vive dentro da bola de raio $\varepsilon$. Para todo $\varepsilon>0$, seja $\operatorname{Int}\left(B_{\varepsilon}\right)$ a bola aberta de raio $\varepsilon$ centrada na origem em $\mathbb{C}^{n}$. Para todo $\eta>0$, sejam $B_{\eta}$ a bola fechada centrada na origem em $\mathbb{C}$ e $\partial B_{\eta}$ sua fronteira. Então, fixado um germe de função analítica $f$, existe $\varepsilon_{0}>0$ tal que, para todo $\varepsilon, 0<\varepsilon<\varepsilon_{0}$, existe $\eta_{\varepsilon}>0$ tal que, para todo $\eta$, $0<\eta<\eta_{\varepsilon}$, a restrição de $f$ como uma aplicação $f: \operatorname{Int}\left(B_{\varepsilon} \cap f^{-1}\left(\partial B_{\eta}\right)\right) \rightarrow B_{\eta}$ é uma fibração suave localmente trivial cujo tipo de difeomorfismo independe da escolha de $\varepsilon$ e $\eta$.

Essa fibração é chamada de fibração de Milnor de $f$ na origem. Denotaremos a fibra por $F_{f}$.

O número de Milnor, que definiremos a seguir, é um invariante de suma importância na teoria de singularidades, auxiliando na teoria de classificação.

Definição 1.22: Sejam $f \in \mathcal{O}_{n}$, germe de função analítica com singularidade isolada na origem, $e$ $J(f)$ o ideal jacobiano de $f$. Definimos o número de Milnor $\mu$ como $\operatorname{dim}_{\mathbb{C}} \frac{\mathcal{O}_{n}}{J(f)}$.

Teorema 1.23: Seja $f \in \mathcal{O}_{n}$ germe de função analítica com singularidade isolada na origem, então cada fibra $F_{f}$ tem o mesmo tipo de homotopia de um buquê de $S^{n-1}$ esferas, e o número de esferas do buquê é exatamente $\mu$.

\subsection{Variedades Algébricas}

Trabalharemos aqui com o caso complexo. O $n$-espaço afim sobre $\mathbb{C}$, denotado por $\mathbb{A}^{n}$ é o conjunto de todas a $n$-uplas de elementos de $\mathbb{C}$. Um ponto $p \in \mathbb{A}^{n}$ é dado por $p=\left(a_{1}, \ldots, a_{n}\right)$, com $a_{i} \in \mathbb{C}$, 
e $a_{i}$ a $i$-ésima coordenada de $p$.

Seja $\mathbb{C}\left[x_{1}, \cdots, x_{n}\right]$ o anel de polinômios em $n$ variáveis sobre $\mathbb{C}$. Podemos interpretar os elementos de $\mathbb{C}\left[x_{1}, \cdots, x_{n}\right]$ como funções do espaço afim $\mathbb{A}^{n}$ sobre $\mathbb{C}$, com $f(p)=f\left(a_{1}, \ldots, a_{n}\right)$, onde $f \in$ $\mathbb{C}\left[x_{1}, \cdots, x_{n}\right]$ e $p \in \mathbb{A}^{n}$. Desta forma, faz sentido falar no conjunto de zeros de $f$, que denotaremos por $Z(f)=\left\{p \in \mathbb{A}^{n} ; f(p)=0\right\}$. De uma forma mais geral, se $T$ for um subconjunto de $\mathbb{C}\left[x_{1}, \cdots, x_{n}\right]$, podemos definir o conjunto de zeros de $T$ como sendo o conjunto de zeros comuns a todos os elementos de $T$. Denotaremos esse conjunto da seguinte forma,

$$
Z(T)=\left\{p \in \mathbb{A}^{n} ; f(p)=0 \forall f \in T\right\} .
$$

Claramente se $I$ for o ideal de $\mathbb{C}\left[x_{1}, \cdots, x_{n}\right]$ gerado por $T$ então $Z(T)=Z(I)$. Mais ainda, como $\mathbb{C}\left[x_{1}, \cdots, x_{n}\right]$ é um anel Noetheriano, qualquer ideal $I$ tem um número finito de geradores $f_{1}, \ldots, f_{r}, \operatorname{logo} Z(T)$ pode se expressar como o conjunto de zeros comuns de um número finito de polinômios $f_{1}, \ldots, f_{r}$.

Definição 1.24: Um subconjunto $Y$ de $\mathbb{A}^{n}$ é chamado conjunto algébrico se existe um subconjunto $T \subset \mathbb{C}\left[x_{1}, \cdots, x_{n}\right]$ tal que $Y=Z(T)$.

Proposição 1.25: A união de dois conjuntos algébricos é um conjunto algébrico. A intersecção de uma família arbitrária de conjuntos algébricos também é um conjunto algébrico.

Para a demonstração deste resultado e referência básica para o assunto ver [34].

Definição 1.26: Os conjuntos algébricos são os conjuntos fechados de uma topologia de $\mathbb{A}^{n}$, denominada topologia de Zariski.

Definição 1.27: Dizemos que $Y \subset X$, um subconjunto não vazio $Y$ contido em $X$ é irredutível se o mesmo não pode se expressar como uma união $Y=Y_{1} \cap Y_{2}$, onde $Y_{1}, Y_{2}$ são fechados em $Y$.

Definição 1.28: Uma variedade algébrica afim é um subconjunto irredutivel de $\mathbb{A}^{n}$ (com a topologia induzida).

\subsection{Espaços Analíticos Complexos}

Analogamente ao caso de polinômios, podemos também estudar o conjunto de zeros de uma ou mais funções analíticas. Estes são os chamados espaços analíticos.

Primeiramente consideremos a seguinte definição:

Definição 1.29: Consideremos o conjunto de pares $\left(V_{\alpha}, U_{\alpha}\right)$, onde $U_{\alpha}$ é uma vizinhança aberta da origem em $\mathbb{C}^{n}$ e $V_{\alpha}$ subconjuntos de $U_{\alpha}$. Dois tais pares $\left(V_{1}, U_{1}\right)$ e $\left(V_{2}, U_{2}\right)$ são equivalentes se existe uma vizinhança $W \subset U_{1} \cap U_{2}$ da origem tal que $V_{1} \cap W=V_{2} \cap W$. Uma classe de equivalência destes pares é chamada germe na origem em $\mathbb{C}^{n}$.

Se $f \in \mathcal{O}_{n}$, a classe de equivalência do conjunto $\{x: f(x)=0\}$, onde $f$ é um representante do germe $f$, é denotada por $\mathcal{V}(f)$; se $f_{1}$ e $f_{2}$ são dois representantes de um mesmo germe, então os conjuntos $\mathcal{V}\left(f_{1}\right)$ e $\mathcal{V}\left(f_{2}\right)$ são iguais. 
Definição 1.30: Um germe de espaço analítico $(V, 0)$ em torno da origem é o germe do subconjunto

$$
V=\mathcal{V}\left(f_{1}\right) \cap \ldots \cap \mathcal{V}\left(f_{r}\right)=\mathcal{V}\left(f_{1}, \ldots, f_{r}\right),
$$

para $f_{1}, \ldots, f_{r} \in \mathcal{O}_{n}$.

Definimos o ideal de um germe de espaço analítico $V$ por

$$
\mathcal{I}(V)=\left\{f \in \mathcal{O}_{n}: V \subset f^{-1}(0)\right\} .
$$

Dizemos que um germe de espaço analítico $V$ é irredutivel quando para quaisquer germes $V_{1} e$ $V_{2}$ tais que $V=V_{1} \cup V_{2}$ então $V=V_{1}$ ou $V=V_{2}$, neste caso dizemos que $V$ é uma variedade analítica.

O nosso objetivo é estudar a natureza de tais espaços analíticos na vizinhança de algum ponto fixado em $\mathbb{C}^{n}$, o qual sem perda de generalidade consideraremos a origem. Para isso precisamos definir formalmente o conceito de germe de um espaço analítico complexo (veja [32]).

Proposição 1.31: Seja $V$ um germe de espaço analítico, entẫo existem um inteiro positivo $p$ e $V_{1}, \ldots, V_{p}$ variedades irredutiveis, com $V_{i}$ não contida em $V_{j}$, para todo $i \neq j$, tais que $V=V_{1} \cup$ $\ldots \cup V_{p}$. Essas variedades são unicamente determinadas, a menos da ordem, e são chamadas de componentes irredutiveis de $V$.

Chamamos de germe de espaço analítico em $x$, um germe de conjunto $V$ em $x$ tal que, para alguma vizinhança $U$ de $x$, o germe $V \cap U$ pode ser descrito por $\mathcal{V}\left(f_{1}, \ldots, f_{r}\right)$, para alguns $f_{1}, \ldots, f_{r} \in$ $\mathcal{O}_{n}$.

Dizemos que um ponto $z$ de um germe de espaço analítico $V$ é um ponto regular ou suave se para alguma vizinhança $U$ de $z$, o germe $U \cap V$ pode ser descrito como o conjunto dos zeros de um número finito de germes de funções analíticas que possuem $z$ como ponto regular. Um ponto de $V$ não regular é chamado de ponto singular de $V$.

Teorema 1.32 (Hilbert's Nullstellensatz - versão local,[27]): Seja I um ideal de $\mathcal{O}_{n}$. Então $\mathcal{I}(\mathcal{V}(I))=$ $\operatorname{Rad}(I)$, onde $\operatorname{Rad}(I)$ é o ideal radical de $I$, ou seja, o ideal $\left\{f \in \mathcal{O}_{n}: \exists n \in \mathbb{N}\right.$ tal que $\left.f^{n} \in I\right\}$.

\subsection{Estratificação de Whitney}

Introduziremos aqui a noção de estratificação de Whitney, introduzida por Whitney [76] e amplamente utilizada desde então. Uma referência para este assunto é [22].

Definição 1.33: Sejam $M$ uma variedade suave e $V \subset M$. Uma estratificação localmente finita de $V$ é uma partição de $V$ em subvariedades de $M$ (chamadas de estratos) tais que, para todo ponto de $V$ existe uma vizinhança em $M$ que encontra apenas um número finito de estratos.

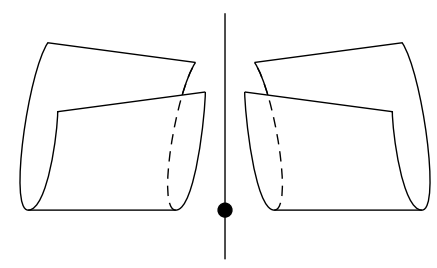


Definição 1.34: Dizemos que a estratificação $\left\{V_{\alpha}\right\}$ de $V$ satisfaz à condição de fronteira, se para dois estratos $V_{\alpha}$ e $V_{\beta}$, tais que $V_{\alpha} \cap \bar{V}_{\beta} \neq \emptyset$ então $V_{\alpha} \subset \bar{V}_{\beta}$.

\section{Condições de Whitney}

Definição 1.35: A estratificação $\left\{V_{\alpha}\right\}$ satisfaz às condições de Whitney se para todo par $\left(V_{\alpha}, V_{\beta}\right)$ de estratos, tais que $V_{\beta}$ esteja no fecho de $V_{\alpha}$ e para todo ponto y de $V_{\beta}$ temos:

a) Para toda seqüência de pontos $x_{i}$ de $V_{\alpha}$ convergindo para $y$, tal que o limite

$$
\lim _{i \rightarrow \infty} T_{x_{i}}\left(V_{\alpha}\right)=T
$$

existe na Grassmanniana correspondente, então $T$ contém $T_{y}\left(V_{\beta}\right)$.

b) Se além disso temos uma seqüência $y_{i}$ de pontos de $V_{\beta}$ com limite y e tal que o limite de direçôes

$$
\lim _{i \rightarrow \infty} \overline{x_{i} y_{i}}=\lambda
$$

existe no espaço projetivo, então $T$ contém $\lambda$.

Estas são as chamadas condição (a) e condição (b) de Whitney.

Whitney mostrou em seu trabalho [76] que toda variedade analítica complexa admite uma estratificação satisfazendo essas duas condições.

Uma estratificação que satisfaz às condições de Whitney e à condição de fronteira é chamada de estratificação de Whitney, ou estratificação Whitney regular.

Exemplo 1.36: Consideremos como primeiro exemplo o cone $C$ com vértice na origem, e a estratificação $\left\{V_{1}, V_{2}\right\}$, onde $V_{1}$ é uma geratriz do cone e $V_{2}=C \backslash V_{1}$. Neste caso, as condições (a) e (b) de Whitney não são satisfeitas. Para ver isto, basta considerar uma seqüência $\left\{x_{i}\right\}$ de pontos de $C$, situados todos sobre uma geratriz $L$ do cone que não seja $V_{1}$, cujo limite seja a origem, de tal modo que o segmento $\overline{x_{i} y_{i}}$ tenha sempre a mesma direção $\lambda$.

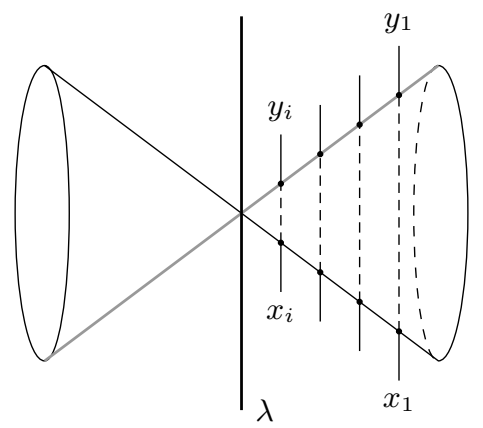

A condição $(a)$ não é satisfeita, já que o limite dos espaços tangentes $T_{x_{i}} L$ não contém o espaço $T_{0}\left(V_{1}\right)$, a verificação de que a condição $(b)$ também não é satisfeita vem do fato que $\lambda$ não está contida no limite dos espaços tangentes $T_{x_{i}} L$. 


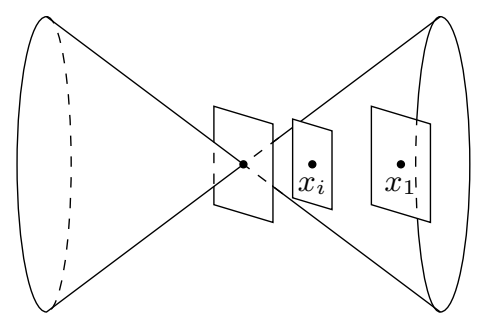

Exemplo 1.37: Consideremos agora a variedade $V$ em $\mathbb{C}^{3}$ dada pelo conjunto de zeros de $y^{2}+x^{3}-$ $t^{2} x^{2}=0$.

Se tomarmos o eixo da coordenada $t$ como um estrato $V_{1}$ e a parte regular da variedade $V_{\text {reg }}$ como sendo outro estrato, temos que a estratificação $\left\{V_{1}, V_{2}\right\}$ satisfaz a condição $(a)$, mas não a condição $(b)$. Porém se acrescentarmos um estrato de dimensão zero, a origem de $\mathbb{C}^{3}$, teremos as duas condições de Whitney satisfeitas.

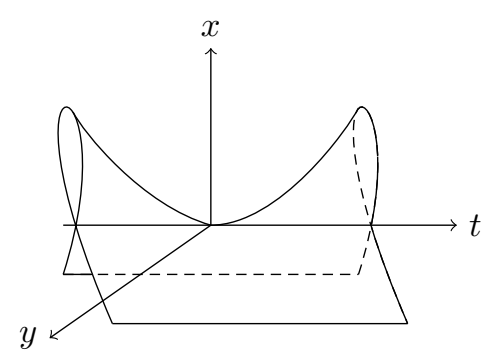

Figura 1.1: Variedade Singular.

\subsection{Modificação de Nash}

A Grassmanniana de $n$-planos de $\mathbb{C}^{m}$ é denotada $G(n, m)$ (para maiores detalhes sobre a variedade Grassmanniana ver [48]). Seja $(V, 0) \subset(M, 0)$ um germe de variedade analítica complexa, equidimensional de dimensão complexa $n$ e $(M, 0)$ um germe de variedade complexa suave de dimensão complexa $m$. Consideremos o fibrado em Grassmannianas dos $n$-planos (complexos) de $T M$, denotado por $G$. A fibra $G_{x}$ sobre $x \in M$ é o conjunto dos $n$-planos de $T_{x} M$, que é isomorfo à $G(n, m)$. Um elemento de $G$ é denotado por $(x, P)$ onde $x \in M$ e $P \in G_{x}$. Sobre a parte suave de $V$, podemos definir a aplicação de Gauss $\phi: V_{\text {reg }} \rightarrow G$ da seguinte forma:

$$
\phi(x)=\left(x, T_{x} V_{r e g}\right)
$$

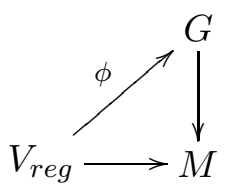

Definição 1.38: A modificação de Nash $\widetilde{V}$ é definida como o fecho da imagem de $\phi$ em G. Ela está munida de uma projeção analítica natural $\nu: \widetilde{V} \rightarrow V$. 
O fibrado tautológico $T$ sobre $G$ é definido da seguinte forma: a fibra $T_{p}$ de $T$, sobre um ponto $p=(x, P) \in G$ é o conjunto de vetores $v$ do $n$-plano $P$.

$$
T_{p}=\left\{v \in T_{x} M: v \in P, x=\nu(x, P)\right\} .
$$

Definimos o fibrado $\widetilde{T}$ de base $\widetilde{V}$ como a restrição de $T$ sobre $\widetilde{V}$, temos então o diagrama:

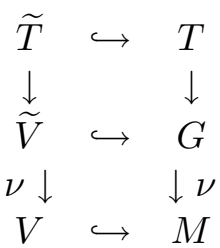

Um elemento de $T$ se escreve como: $(x, P, v)$ onde $P \in G_{x}$ e $v \in P$.

Exemplo 1.39: A transformação de Nash do cone $C$ é um cilindro $\widetilde{C}$.

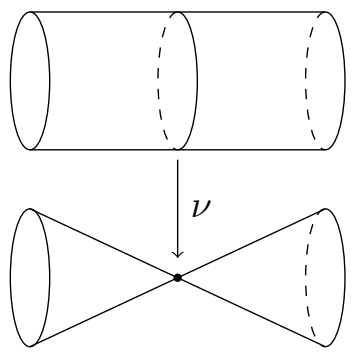

Uma observação importante é o fato que a transformada de Nash de um conjunto singular nem sempre é regular, ou seja, nem sempre a transformada de Nash resolve a singularidade de uma variedade algébrica ou analítica.

Como exemplo ilustrativo, na figura abaixo, a transformada de Nash da curva no plano cinza é a curva espacial, ilustrada na mesma figura, que ainda é singular. Para resultados sobre transformada de Nash e resolução de singularidades sugerimos [64].

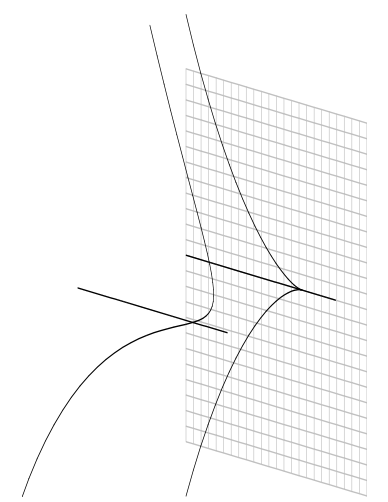

Figura 1.2: A transformada de Nash nem sempre é regular.

\subsection{Variedades Polares}

A base desta seção é um trabalho de divulgação desenvolvido em conjunto com Thiago de Melo sobre exemplos de variedades polares no caso de superfícies [31], o que auxiliou para uma redação mais clara dessa seção em sua versão final. 
O conceito de Variedades Polares foi introduzido por Bernard Teissier e Lê Dũng Tráng nos anos $70([68,45])$. Desde então tem sido uma ferramenta de grande importância em vários domínios da matemática, como Teoria de Singularidades, Teoria de Estratificação, Geometria Algébrica e Classes Características. Uma literatura mais avançada sobre a relação entre Classes Características e Variedades Polares é [6].

Várias abordagens sobre Variedades Polares foram desenvolvidas baseadas em conceitos abstratos, como por exemplo Projeções Genéricas, Modificação de Nash e Variedade Conormal ([20, 68]).

Primeiramente tentaremos introduzir a noção de variedades polares de uma forma intuitiva e simples e para isso trabalharemos inicialmente com superfícies reais, embora posteriormente trabalharemos com variedades analíticas complexas. Utilizaremos no caso de superfícies basicamente Álgebra Linear e Cálculo Diferencial. Isto se torna interessante pois a presença de figuras que ilustram a técnica utilizada nos leva a uma melhor compreensão do assunto.

\section{Variedades Polares}

Trataremos agora de uma simples questão, que nos levará à formalização do conceito de Variedades Polares. Para uma melhor visualização, todos os planos e retas nas figuras a seguir estão transladados, de modo a não se sobreporem.

Consideremos a esfera unitária $S^{2} \subset \mathbb{R}^{3}$, dada por $F^{-1}(0)$, onde $F: \mathbb{R}^{3} \rightarrow \mathbb{R}$ é dada por $F(x, y, z)=x^{2}+y^{2}+z^{2}-1$. Dado um plano $\pi \subset \mathbb{R}^{3}$ contendo a origem, queremos encontrar os pontos de $S^{2}$ com maior ordem de contato com o plano $\pi$, ou seja, pontos $p \in S^{2}$ para os quais $\operatorname{dim}\left(T_{p} S^{2} \cap \pi\right)$ seja a maior possível.

Por exemplo, para $\pi$ dado por $z=0$, encontramos apenas os pontos $(0,0,1)$ e $(0,0,-1)$, já que para qualquer outro ponto $q \in S^{2}$ temos que $T_{q} S^{2} \cap \pi$ é uma reta.

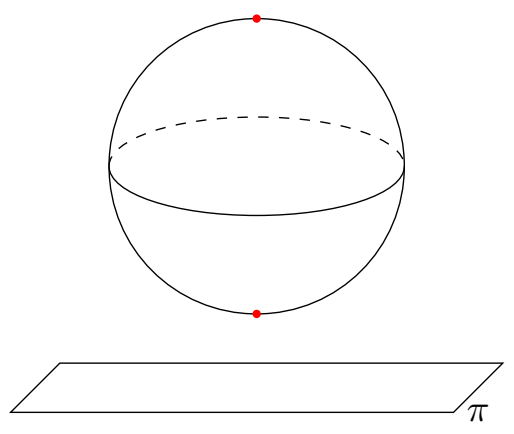

Figura 1.3: Variedade polar $P_{2}\left(S^{2}, \pi\right)$ de codimensão 2.

Isto se deve ao fato da equação geral do plano tangente a $S^{2}$ no ponto $p=\left(x_{0}, y_{0}, z_{0}\right)$ ser

$$
\frac{\partial F}{\partial x}(p) x+\frac{\partial F}{\partial y}(p) y+\frac{\partial F}{\partial z}(p) z=0
$$

ou seja,

$$
2 x_{0} x+2 y_{0} y+2 z_{0} z=0 .
$$

Portanto, basta observar que os planos $T_{p} S^{2}$ e $\pi$ coincidem se, e somente se, $x_{0}=y_{0}=0$ e $z_{0}= \pm 1$.

Uma situação mais interessante surge quando trocamos o plano $\pi$ por uma reta $L$. Neste caso, procuramos por pontos $p \in S^{2}$ tais que $\operatorname{dim}\left(T_{p} S^{2} \cap L\right)=1$, isto é, $L \subset T_{p} S^{2}$. 
Sejam $v=\left(v_{1}, v_{2}, v_{3}\right)$ um vetor diretor de $L$ e $w=\left(\frac{\partial F}{\partial x}(p), \frac{\partial F}{\partial y}(p), \frac{\partial F}{\partial z}(p)\right)$ um vetor normal de $T_{p} S^{2}$. Como a inclusão acima ocorre se, e somente se, $v \perp w$, resolvemos o problema buscando as soluções de

$$
\left\{\begin{array}{l}
F(x, y, z)=0 \\
v_{1} \frac{\partial F}{\partial x}(x, y, z)+v_{2} \frac{\partial F}{\partial y}(x, y, z)+v_{3} \frac{\partial F}{\partial z}(x, y, z)=0
\end{array}\right.
$$

nas variáveis $x, y, z$.

Por exemplo, para $v=(1,1,1)$ temos

$$
\left\{\begin{array}{l}
x^{2}+y^{2}+z^{2}-1=0 \\
2 x+2 y+2 z=0
\end{array}\right.
$$

cuja solução é a intersecção de $S^{2}$ com o plano $2 x+2 y+2 z=0$, isto é, um grande círculo.

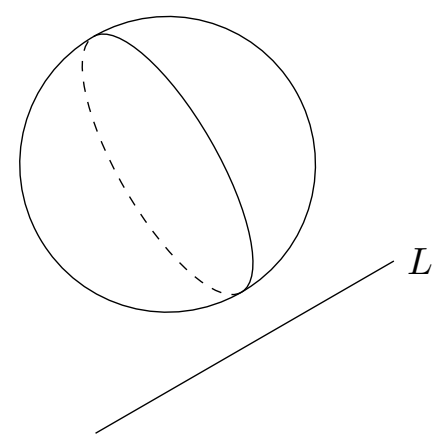

Figura 1.4: Variedade polar $P_{1}\left(S^{2}, L\right)$ de codimensão 1.

Buscando formalizar e também generalizar as idéias acima, necessitamos do conceito de transversalidade.

Lembramos que dois subespaços vetoriais $E, F \subset \mathbb{R}^{N}$ são transversais se $E+F=\mathbb{R}^{N}$. Além disso, valem as igualdades

$$
\begin{gathered}
\operatorname{codim}(E \cap F)=\operatorname{codim}(E)+\operatorname{codim}(F), \\
\operatorname{dim}(E \cap F)=\operatorname{dim}(E)-\operatorname{codim}(F),
\end{gathered}
$$

onde $\operatorname{codim}(E)=N-\operatorname{dim}(E)$.

Caso $E, F \subset \mathbb{R}^{N}$ não sejam transversais, temos

$$
\operatorname{dim}(E \cap F)>\operatorname{dim}(E)-\operatorname{codim}(F),
$$

pois se $\operatorname{dim}(E \cap F)<\operatorname{dim}(E)-\operatorname{codim}(F)$ teríamos

$$
\operatorname{dim}(E)+\operatorname{dim}(F)-\operatorname{dim}(E+F)<\operatorname{dim}(E)-(N-\operatorname{dim}(F)),
$$

isto é, $\operatorname{dim}(E+F)>N$, o que é absurdo.

Resumindo:

$$
\begin{aligned}
E \text { é transversal a } F & \Longleftrightarrow \operatorname{dim}(E \cap F)=\operatorname{dim}(E)-\operatorname{codim}(F) \\
E \text { não é transversal a } F & \Longleftrightarrow \operatorname{dim}(E \cap F)>\operatorname{dim}(E)-\operatorname{codim}(F)
\end{aligned}
$$


O conceito de transversalidade torna-se importante neste momento pois a ocorrência de maior contato entre uma variedade $M$ e um subespaço vetorial $D$, em um dado ponto $p \in M$, equivale a não-transversalidade entre os espaços vetoriais $T_{p} M$ e $D$.

Note que, nos exemplos acima, $D_{1}=\pi$ e $D_{2}=L$, onde o índice representa a codimensão de $D$ em $\mathbb{R}^{3}$. Assim, a não-transversalidade entre $S^{2}$ e $D_{1}$ ocorre se, e somente se,

$$
\operatorname{dim}\left(T_{p} S^{2} \cap D_{1}\right)>\operatorname{dim}\left(T_{p} S^{2}\right)-\operatorname{codim}\left(D_{1}\right)=\operatorname{dim}\left(S^{2}\right)-1=1
$$

Logo, a não-transversalidade equivale a $\operatorname{dim}\left(T_{p} S^{2} \cap D_{1}\right)=2$, já que $\operatorname{dim}\left(T_{p} S^{2}\right)=\operatorname{dim}\left(D_{1}\right)=2$. O cálculo para $D_{2}$ é análogo.

Estamos em condições de introduzir a definição de variedades polares.

Sejam $M^{d} \subset \mathbb{C}^{N}$ (ou $\mathbb{R}^{N}$ ) uma variedade diferenciável de dimensão $d$ e $D_{d-k+1}$ um subespaço vetorial de codimensão $d-k+1$ em $\mathbb{C}^{N}$ ou $\mathbb{R}^{N}$. Definimos a $k$-ésima variedade polar de $M$ com relação a $D_{d-k+1}$ como sendo

$$
P_{k}\left(M, D_{d-k+1}\right)=\left\{p \in M ; \operatorname{dim}\left(T_{p} M \cap D_{d-k+1}\right) \geq k\right\},
$$

onde dim denota a dimensão complexa no caso complexo e dimensão real no caso real. No caso complexo se pode provar que, para todo $k=0,1, \ldots, d, \operatorname{codim}\left(P_{k}\left(M, D_{d-k+1}\right)\right)=k$, para $D_{d-k+1}$ em um aberto denso de $G(N-d+k-1, N)$.

Com o intuito de fixar melhor as idéias discutidas acima, exibiremos mais alguns exemplos, utilizando superfícies reais bem conhecidas.

No caso do toro $T$, obtido por $F^{-1}(0)$, onde

$$
F(x, y, z)=z^{2}+\left(\sqrt{x^{2}+y^{2}}-2\right)^{2}-1,
$$

$P_{2}(T, \pi)$ é exatamente o conjunto dos pontos $p \in T$ tais que o vetor normal a $T$ em $p$ é múltiplo do vetor normal de $\pi$. Isto se torna claro nas figuras abaixo.

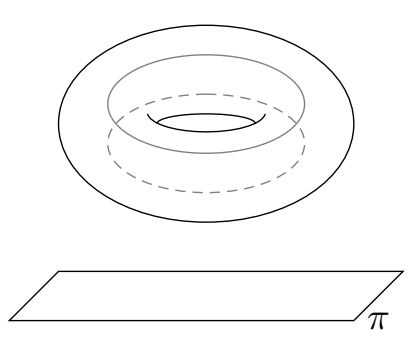

(a)

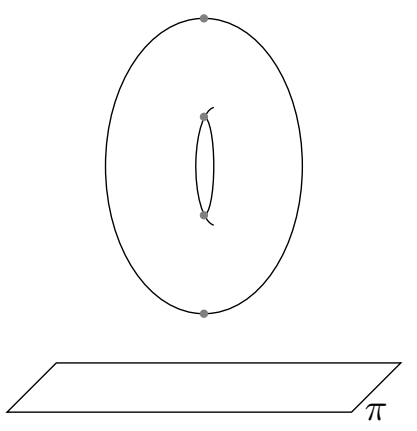

(b)

Figura 1.5: (a) $P_{1}\left(T, \pi_{1}\right)$, (b) $P_{2}\left(T, \pi_{2}\right)$.

Note que na figura (1.5a), a variedade polar não possui codimensão 2. Isto ocorre pois o plano $\pi_{1}$ não pertence ao aberto denso citado acima. 


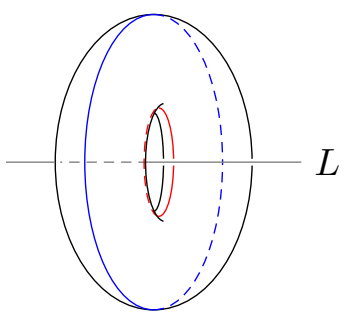

(a)

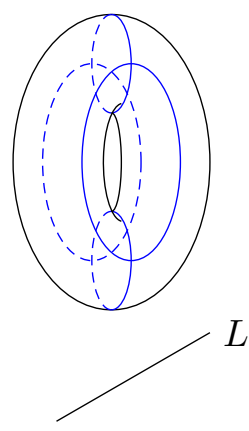

(b)

Figura 1.6: (a) $P_{1}\left(T, L_{1}\right)$, (b) $P_{1}\left(T, L_{2}\right)$.

Para exibirmos $P_{1}(T, L)$, encontramos as soluções de (1.1) com o auxílio do computador e obtemos duas configurações possíveis, representadas na figura abaixo, para as retas $L_{1}$ e $L_{2}$.

Note que, apesar das codimensões de $P_{1}(T, L)$ serem iguais em (1.6a) e (1.6b), para pequenas perturbações de $L_{2}$ obtemos duas curvas como em (1.6a) e não mais quatro como em (1.6b). Isto se deve ao fato da reta $L_{2}$ não pertencer ao aberto denso, citado acima.

Em [31] apresentamos um algoritmo para o Maple, dependendo apenas de $v=\left(v_{1}, v_{2}, v_{3}\right)$, que permite uma visualização dos conceitos acima.

Utilizando este algoritmo com os valores especificados abaixo, obtemos a figura (1.7a), mostrando o toro $T$, a reta $L$ cujo vetor diretor é $v=\left(1, \frac{1}{2}, \frac{3}{5}\right)$ e a superfície dada implicitamente pela equação

$$
2\left[\frac{\left(\sqrt{x^{2}+y^{2}}-2\right)\left(x+\frac{1}{2} y\right)}{\sqrt{x^{2}+y^{2}}}+\frac{3}{5} z\right]=0 .
$$

A variedade polar $P_{1}(T, L)$ é a intersecção destas duas superfícies, mostrada na figura (1.7b). O leitor pode alterar as coordenadas de $v$, movimentar facilmente as superfícies, alterar as cores, entre outros recursos, podendo assim ter uma melhor visualização, como por exemplo em (1.7c), obtida usando $v=\left(1, \frac{1}{2}, \frac{1}{10}\right)$. Quando $v_{3}=0$, temos um "caso limite", mostrado em (1.7d) e (1.7e).

Aqui percebemos que algumas variedades mantêm sua configuração e outras não, porém as que não mantêm essa configuração, quando perturbadas, caem no caso que chamaremos de genérico.

\section{Variedades Polares e Projeções Lineares}

Uma outra definição para variedades polares pode ser dada em função de uma projeção genérica, que em termos gerais significa ser a projeção menos singular possível. Esta é a definição usada por autores como T. Gaffney, V. H. Jorge-Perez, Lê D. T., D. Levcovitz, M. J. Saia, B. Teissier e etc [20, 38, 45, 68]. Uma definição mais precisa é apresentada no trabalho de B. Teissier (ref. [68]).

Se pensarmos em $D_{d-k+1}$ como núcleo de uma projeção linear, podemos redefinir a variedade polar da seguinte forma:

Proposição 1.40: Seja $\Pi: \mathbb{C}^{N} \rightarrow \mathbb{C}^{d-k+1}$ uma projeção linear, $\operatorname{Ker}(\Pi)=D_{d-k+1}$. A k-ésima variedade polar de uma variedade regular $M$, com relação à $D_{d-k+1}$, que indicamos por $P_{k}\left(V, D_{d+k+1}\right)$, coincide com o conjunto de pontos críticos da projeção $P$ restrita à variedade $M$. 


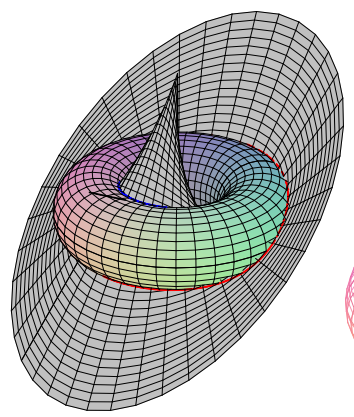

(a)

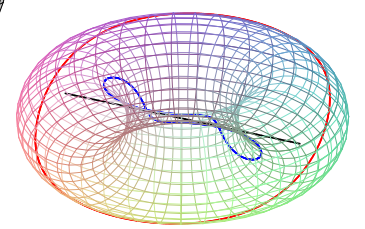

(b)

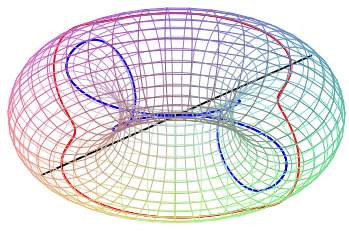

(c)

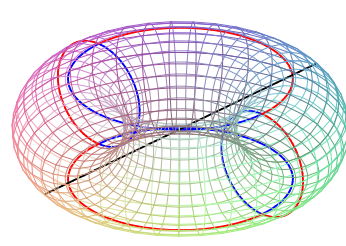

(d)

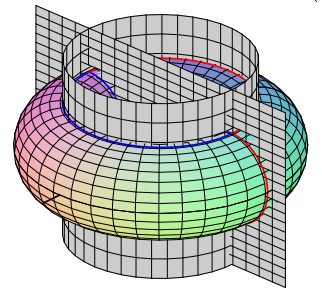

(e)

Figura 1.7: Variedade polar $P_{1}(T, L)$ de codimensão 1.

Demonstração: Se $p \in \Sigma\left(\left.P\right|_{M}\right)$ então temos que $\operatorname{dim}\left(\Pi\left(T_{p} M\right)\right)<d-k+1$, ou seja, $\operatorname{dim}\left(T_{x} M\right)-$ $\operatorname{dim}\left(\operatorname{Ker}\left(\left.\Pi\right|_{M}\right)\right)<d-k+1$, logo temos $\operatorname{dim}\left(T_{p} M \cap D_{d-k+1}\right)>k-1$, que podemos escrever com $\operatorname{dim}\left(T_{p} M \cap D_{d-k+1}\right)>k-1$, de onde segue o resultado.

No caso de uma variedade singular algébrica ou analítica, equidimensional, reduzida, definimos a variedade polar da seguinte forma:

Definição 1.41 ([45]): Seja $\left(V^{d}, 0\right)$ um germe de variedade algébrica (resp. analítica), reduzida e equidimensional. Sejam $V$ um representante suficientemente pequeno do germe, denotemos por $V_{\text {reg }}$ a parte regular de $V$. Definimos $P_{k}\left(V, D_{d-k+1}\right), 0 \leq k \leq d$ como sendo:

$$
P_{k}\left(V, D_{d+k+1}\right)=\overline{P_{k}\left(V_{r e g}, D_{d-k+1}\right)}
$$

onde $V_{\text {reg }}$ denota a parte regular de $V$.

Exemplo 1.42: Consideremos a superfície $V=\left\{(x, y, t) \in \mathbb{C}^{3} ; F(x, y, z)=y^{2}-x^{3}+t^{2} x^{2}=0\right\}$ e a projeção paralela ao eixo-y. A parte singular de $V$ é o eixo-t. Seja $\left(x_{0}, y_{0}, t_{0}\right) \in V_{\text {reg }}$, o plano tangente à superfície nesse ponto é dado por,

$$
x \partial_{x} F\left(x_{0}, y_{0}, t_{0}\right)+y \partial_{y} F\left(x_{0}, y_{0}, t_{0}\right)+z \partial_{z} F\left(x_{0}, y_{0}, t_{0}\right)=0 .
$$

Para que a projeção $\Pi$ restrita ao plano tangente $T_{x \partial_{x} F\left(x_{0}, y_{0}, t_{0}\right)} V_{\text {reg }}$ não seja uma aplicação sobrejetora, o plano tangente deve conter a eixo- $y$, ou seja, pontos do tipo $(0, y, 0)$ devem satisfazer a equação do plano, logo queremos $\partial_{y} F x \partial_{x} F\left(x_{0}, y_{0}, t_{0}\right)=0$.

Então temos que a variedade polar $P_{1}(V, P)$ associada à projeção $\Pi$ é dada como fecho da solução do sistema abaixo,

$$
\left\{\begin{aligned}
\partial_{y} F(x, y, t) & =0 \\
F(x, y, t) & =0
\end{aligned} \quad \operatorname{com}(x, y, t) \in V_{\text {reg }}\right.
$$


e portanto

$$
\left\{\begin{array}{r}
2 y=0 \\
y^{2}-x^{3}+t^{2} x^{2}=0
\end{array} \quad \operatorname{com}(x, y, t) \in V_{\text {reg }} .\right.
$$

Ou seja $\overline{\left\{(x, y, t) \in V_{\text {reg }} ; x^{2}\left(x-t^{2}\right)=0\right\}}$.

\section{Ciclos de Schubert}

Introduziremos aqui os ciclos de Schubert, os quais têm uma relação estreita com as variedades polares que são usadas neste trabalho.

Definição 1.43: Seja $D_{d-k+1}$ um subespaço vetorial de $\mathbb{C}^{N}$ de codimensão $d-k+1$, definimos,

$$
C\left(D_{d-k+1}\right)=\left\{T \in G_{d}\left(\mathbb{C}^{N}\right) ; \operatorname{dim}\left(T \cap D_{d-k+1}\right) \geq k\right\}
$$

Consideremos o diagrama abaixo,

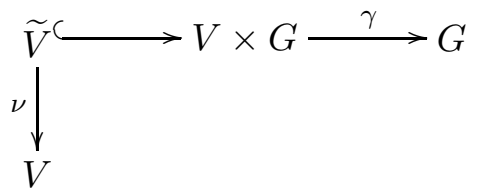

onde $\Phi(z, T)=(z, T), \gamma(z, T)=T$ e $\widetilde{V}$ a modificação de Nash de $V$.

Temos então a seguinte proposição:

Proposição 1.44: A variedade polar pode ser expressa em termos do diagrama acima da seguinte forma: $P_{k}\left(V_{\text {reg }}, D_{d-k+1}\right)=\nu\left(\Phi^{-1}\left(\gamma^{-1}\left(C\left(D_{d-k+1}\right)\right)\right) \cap \Phi^{-1}(V \backslash \Sigma(V))\right)$, e logo temos que,

$$
P_{k}\left(V, D_{d-k+1}\right)=\overline{\nu\left(\Phi^{-1}\left(\gamma^{-1}\left(C\left(D_{d-k+1}\right)\right)\right) \cap \Phi^{-1}(V \backslash \Sigma(V))\right)} .
$$

Demonstração: É fácil ver que

$$
\begin{aligned}
\nu\left(\Phi^{-1}\left(\gamma^{-1}\left(C\left(D_{d-k+1}\right)\right)\right) \cap \Phi^{-1}(V \backslash \Sigma(V))\right) & =\left\{x \in V_{\text {reg }} ;\left(x, T_{x} V_{\text {reg }}\right) \in \gamma^{-1}\left(D_{d-k+1}\right)\right\} \\
& =\left\{x \in V_{\text {reg }} ; \operatorname{dim}\left(T_{x} V_{\text {reg }} \cap D_{d-k+1}\right) \geq k\right\} \\
& =P_{k}\left(V_{\text {reg }}, D_{d-k+1}\right),
\end{aligned}
$$

o que implica o resultado.

\subsection{Multiplicidades Polares}

Multiplicidade de um ponto sobre uma curva

Definição 1.45: A multiplicidade de um ponto $p$ sobre uma curva plana $C$, denotada por $m_{p}(C)$ é o número de intersecção da curva com um reta secante genérica na vizinhança do ponto. 


\section{Multiplicidade de um ponto sobre uma subvariedade}

Definição 1.46: Seja $V$ uma subvariedade de dimensão $k$ contida em uma variedade regular $M$.

Seja $D$ um pequeno polidisco centrado em $p$. Consideremos a projeção $P$ sobre um plano genérico de dimensão $k$. Seja q um ponto de $P(D) \backslash\{P(p)\}$ e $\delta(p)$ um pequeno polidisco centrado em $q$. A multiplicidade de $V$ em $p$, denotada por $m_{p}(V)$ é o número de folhas de $P^{-1}(\delta) \cap D \cap V$.

Existe uma forma algébrica de calcular essa multiplicidade [77].

Considere a seguinte função sobre $\mathbb{N}$.

$$
F(k)=\operatorname{dim}_{\mathbb{C}} \mathcal{O}_{V, x} / \mathfrak{M}_{V, x}^{k+1},
$$

onde $\mathfrak{M}_{V, x}$ denota o ideal maximal de $\mathcal{O}_{V, x}$. Esta função é chamada de função de Samuel de $\mathcal{O}_{V, x}$. Para $k>>0$ a função $F$ se comporta como um polinômio em $k$ de grau $n-1$ com termo de maior ordem igual à $\frac{e(V, x)}{(n-1)} k^{n-1}$, e o numerador do coeficiente do termo de maior ordem $e(V, x)$ é chamado de multiplicidade de Hilbert-Samuel de $V$ em $x$.

Teissier usou esses invariantes para dar uma condição necessária e suficiente para uma estratificação ser Whitney regular através do seguinte resultado:

Teorema 1.47: Sejam $V$ uma variedade analítica reduzida, equidimensional de dimensão d, $Y$ um subespaço analítico de $V$ também equidimensional, e 0 um ponto não singular de $Y$. Então as seguintes condiçôes são equivalentes:

1. A aplicação de $Y$ em $\mathbb{N}^{d}$, definida por

$$
y \mapsto\left(m_{y}(V), m_{y}\left(P_{1}(V, y)\right), \cdots, m_{y}\left(P_{d-1}(V, y)\right)\right)
$$

é constante numa vizinhança da origem.

2. O par de estratos $(V \backslash Y, Y)$ satisfaz às condições $(a)$ e (b) de Whitney na origem.

Demonstração: A demonstração deste resultado se encontra em [68].

Exemplo 1.48: Calculemos as multiplicidades polares da variedade singular

$$
V=\left\{(x, y, t) \in \mathbb{R}^{3} ; y^{2}-x^{3}-t^{2} x^{2}=0\right\} .
$$

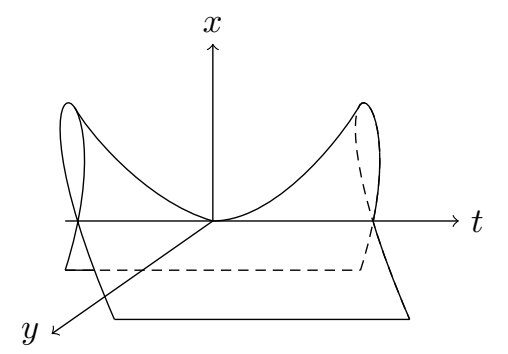

Figura 1.8: Variedade Singular.

Neste caso, $P_{0}\left(V, D_{2}\right)=V$ e $P_{1}\left(V, D_{1}\right)=\left\{(x, y, t) \in \mathbb{R}^{3} ; x=-t^{2}, y=0\right\}$, onde $D_{2}$ é o espaço vetorial nulo e $D_{1}$ é a reta definida pelo eixo-y, de onde obtemos $m_{0}(V, 0)=2$ e $m_{1}(V, 0)=1$. 


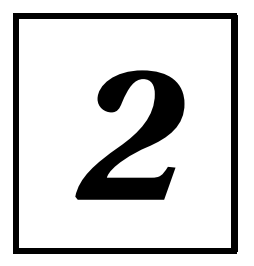

\section{Obstrução de Euler Local}

Neste capítulo estudaremos a obstrução de Euler local definida por MacPherson [49] como um dos ingredientes da sua prova da conjectura de Deligne e Grothendieck. Esta conjectura trata da existência e unicidade das classes características de variedades algébricas complexas singulares. Uma referência básica sobre o desenvolvimento da obstrução de Euler local é o artigo de J.-P > Brasselet (ref. [5]).

Existe também uma definição para esta obstrução devida a J.-P. Brasselet e M.-H. Schwartz [10], que será considerada neste trabalho. Gonzales-Sprinberg e Verdier [24] mostraram uma fórmula para o obstrução de Euler local em função das classes de Chern de fibrados vetoriais sobre a transformada de Nash da variedade algébrica. Neste capítulo estudaremos uma fórmula de Lê D. T. e B. Teissier [45], em que a obstrução de Euler se exprime em termos de invariantes polares. Em [7], J.-P. Brasselet, Lê D. T. e J. Seade apresentam uma fórmula para esta obstrução em termos da característica de Euler da interseção da fibra de $f$ com uma hipersuperfície genérica e os estratos de uma estratificação de Whitney. Esta é uma fórmula do tipo Lefschetz, muito importante no desenvolvimento do nosso trabalho.

\subsection{Obstrução de Euler Local}

Sejam $(V, 0) \subset\left(\mathbb{C}^{n}, 0\right)$ germe de variedade analítica como anteriormente, $V$ um representante suficientemente pequeno do germe e $\left\{V_{\alpha}\right\}$ uma estratificação de Whitney de uma vizinhança da origem $U$, compatível com $V$. Suponhamos também que a origem $\{0\}$ seja um estrato. Seja $\cup T V_{\alpha}$ a união dos fibrados tangentes a todos os estratos. Podemos olhar esta união como um subconjunto do fibrado tangente $\left.T \mathbb{C}^{n}\right|_{V}$.

Definição 2.1: Seja $v$ uma seção de $T \mathbb{C}^{n}$ sobre um subconjunto $A$ de $\mathbb{C}^{n}$. Dizemos que o campo $v$ é estratificado se para $z \in V_{\alpha} \cap A$, temos $v(z) \in T_{z} V_{\alpha}$, onde $V_{\alpha}$ é o estrato que contém $z$.

Seja $B_{\epsilon}(a)$ a bola em $\mathbb{C}^{n}$ centrada em $a$ e de raio $\epsilon$.

Definição 2.2: Dizemos que o campo de vetores $v$ é radial em a se $v$ é um campo estratificado e se existe $\epsilon_{0}>0$ tal que o campo $v$ está saindo de $B_{\epsilon}(a)$, para $0<\epsilon \leq \epsilon_{0}$. 
Lema 2.3 ([10], Prop. 9.1): Um campo de vetores estratificado $v$ não nulo definido sobre um subconjunto $A \subset V$ admite um levantamento canônico $\tilde{v}$ como seção não nula de $\widetilde{T}$ sobre $\nu^{-1}(A)$.

Demonstração: Para um ponto $\tilde{z}$ de $\tilde{V}$ tal que $z=\nu(\tilde{z})$, com $z \in V_{\text {reg }} \operatorname{definimos} \tilde{v}(\tilde{z}) \in \widetilde{T}$ por $\tilde{v}(\tilde{z})=(z, P, v)$, onde $P=T_{z}\left(V_{\text {reg }}\right)$.

Se $\tilde{z}$ de $\tilde{V}$ é tal que $z=\nu(\tilde{z})$ é ponto singular de $V$, temos $P=\lim _{z_{i} \rightarrow z} T_{z_{i}\left(V_{\text {reg }}\right)}$ onde $\left(z_{i}\right)$ é uma seqüência de pontos de $V_{\text {reg }}$ tendendo para $z, v(z) \in T_{z}\left(V_{\alpha}\right)$ onde $V_{\alpha}$ é o estrato que contém $z$. Pela condição $(a)$ de Whitney $T_{z}\left(V_{\alpha}\right) \subset P$, então $v \in P$. Podemos então definir $\tilde{v}(\tilde{z}) \in \widetilde{T}$ por $\tilde{v}(\tilde{z})=(z, P, v)$.

Temos então o seguinte diagrama :

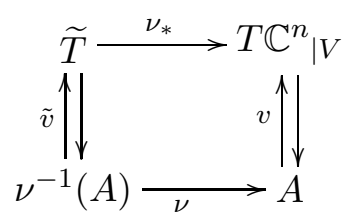

Se $\tilde{z}$ é um ponto de $\widetilde{V}$ tal que $\nu(\tilde{z})=z$, então $v(z) \in T_{z}\left(V_{\alpha}\right) \subset T_{z} \mathbb{C}^{n}$. Temos então $\nu_{*}(\tilde{v}(\tilde{z}))=$ $v(z)$.

Teorema 2.4 (Bertini-Sard, ver [73]): Se $\left\{V_{\alpha}\right\}$ é uma estratificação de Whitney de $V$, para $\epsilon$ suficientemente pequeno (e positivo) a esfera $\partial B_{\epsilon}(0)$ é transversal aos estratos $V_{\alpha}$.

Denotaremos $B_{\epsilon}=B_{\epsilon}(0)$.

A obstrução de Euler local foi definida por MacPherson em [49], a definição a seguir é atribuída à J.-P. Brasselet e M.-H. Schwartz ([10], Prop. 10.1).

Definição 2.5: Seja $v$ um campo de vetores radial sobre $V \cap \partial B_{\epsilon}$ e $\tilde{v}$ o levantamento de $v$ sobre o conjunto $\nu^{-1}\left(V \cap \partial B_{\epsilon}\right)$. O campo $\tilde{v}$ define um cociclo de obstrução Obs $(\tilde{v})$, que mede a obstrução para estender $\tilde{v}$ como seção não-nula de $\widetilde{T}$ sobre $\nu^{-1}\left(V \cap B_{\epsilon}\right)$ :

$$
\operatorname{Obs}(\tilde{v}) \in Z^{2 n}\left(\nu^{-1}\left(V \cap B_{\epsilon}\right), \nu^{-1}\left(V \cap \partial B_{\epsilon}\right)\right) .
$$

A obstrução de Euler local EuV(0) é a avaliação do cociclo Obs(v) sobre a classe fundamental $\left[\nu^{-1}\left(V \cap B_{\epsilon}\right), \nu^{-1}\left(V \cap \partial B_{\epsilon}\right)\right]$, ou seja :

$$
E u_{V}(0):=\left\langle\operatorname{Obs}(\tilde{v}),\left[\nu^{-1}\left(V \cap B_{\epsilon}\right), \nu^{-1}\left(V \cap \partial B_{\epsilon}\right)\right]\right\rangle .
$$

\subsection{Fórmula de Gonzalez-Sprinberg e Verdier}

Gonzalez-Sprinberg e Verdier obtêm em [24] uma fórmula para a obstrução de Euler local, em função das classes de Chern de fibrados vetoriais sobre a transformada de Nash da variedade algébrica. Marcos Sebastiani em [62] demonstra este mesmo resultado, mas adotando um ponto de vista mais topológico, o que deixa a demonstração consideravelmente mais curta e mais compreensível e esta é a demonstração que apresentaremos aqui. 


\section{Divisores}

Seja $V$ uma variedade complexa de dimensão $d$. Qualquer subvariedade analítica $W \subset V$ de dimensão $d-1$ é uma hipersuperfície analítica, ou seja, se $p \in W \subset V, W$ pode ser dada localmente em torno de $p$ como conjunto de zeros de apenas uma função analítica. Mais ainda, qualquer outra função analítica definida em uma vizinhança de $p$ que se anule em $W$ é divisível por $f$. É fácil ver que $W$ pode se expressar unicamente como união de hipersuperfícies analíticas irredutíveis.

$$
W=V_{1} \cup V_{2} \cup \cdots \cup V_{k},
$$

Definição 2.6: Um divisor de Weil $D$ em $V$, aqui chamado apenas de divisor em $V$, é uma combinação linear formal de hipersuperfícies analíticas irredutiveis de $V$,

$$
D=\sum a_{i} V_{i}
$$

Um divisor $D=\sum a_{i} V_{i}$ é chamado efetivo se $a_{i} \geq 0$ para todo $i$; e escrevemos $D \geq 0$. O Conjunto dos divisores de $V$ forma um grupo aditivo, denotado por $\operatorname{Div}(V)$.

\section{Classe fundamental de um divisor}

Seja $V$ um espaço analítico reduzido de dimensão $d$ e seja $D$ um divisor de $V$. Suponhamos que $D$ seja efetivo e com suporte compacto.

Seja $E$ o fibrado vetorial de posto complexo 1 associado a $D$. Como $D$ é efetivo, $E$ está canonicamente munido de uma seção não-trivial $s$. O conjunto de zeros de $s$ é o suporte $|D|$ de D. Então podemos considerar,

$$
s:(V, V \backslash|D|) \rightarrow\left(E, E^{*}\right),
$$

onde $E^{*}$ é o complementar da seção nula em $E$.

Seja $\omega$ a classe de Thom de $E, \omega \in H^{2}\left(E, E^{*} ; Z\right)$.

Definição 2.7: A classe $[D]=s^{*}(\omega) \cap[V] \in H_{2 N-2}(|D| ; Z)$ é chamada classe fundamental do divisor $D([V]$ denota a classe fundamental de $V)$.

Sejam $D_{1}, \cdots, D_{r}$ as componentes irredutiveis de D. Então, podemos descrever de forma única: $[D]=\sum n_{j}\left[D_{j}\right]$, que é denominado ciclo associado a $D$.

Lema 2.8: Sejam $E \rightarrow V$ um fibrado real orientado de posto $d, V$ um complexo finito, $Y \subset V$ um sub-complexo. Seja $s: V \rightarrow E$ uma seção tal que $s(y) \neq 0$ para todo $y \in Y$. Seja $\omega \in H^{d}\left(E, E^{*} ; Z\right)$ a classe de Thom. Então, $s:(V, Y) \rightarrow\left(E, E^{*}\right)$ e $s^{*}(\omega) \in H^{d}(V, Y ; Z)$ é a obstrução para estender $s \mid Y$ à uma seção de $E^{*}$ sobre $V$.

\section{Teorema de Gonzalez-Sprinberg e Verdier}

Consideremos $(V, 0) \subset\left(\mathbb{C}^{n+1}, 0\right)$ germe de variedade analítica, equidimensional de dimensão complexa $d$ e denotaremos a Grassmaniana dos $d$-planos em $\mathbb{C}^{n+1}$ da seguinte como $G=G(d, n+1)$.

Denotaremos por $V^{\prime}$ o fecho do gráfico da aplicação que a cada ponto $x$ de $V \backslash\{0\}$ associa a reta $\overline{0 x} \in \mathbb{P}^{n}$. A aplicação $e: V^{\prime} \rightarrow V$ é a restrição a $V^{\prime}$ da projeção natural $V \times \mathbb{P}^{n} \rightarrow V$. 
De forma semelhante, denotaremos por $\mathfrak{X}$ o fecho do gráfico da aplicação que a cada ponto $x$ de $V_{\text {reg }} \backslash\{0\}$ associa o par formado pela reta $\overline{0 x} \in \mathbb{P}^{n}$ e espaço tangente à $V_{\text {reg }}$ no ponto $x$. Analogamente ao caso anterior, $\tilde{e}: \mathfrak{X} \rightarrow \widetilde{V}$ é a restrição a $\mathfrak{X}$ da projeção natural $V \times \mathbb{P}^{n} \times G \rightarrow V$.

Antes de enunciarmos o resultado observemos o seguinte diagrama:

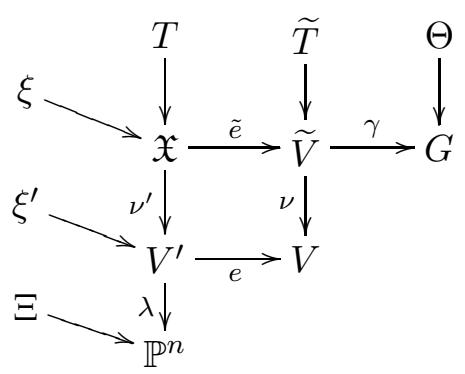

Denotemos $Y^{\prime}=e^{-1}(0), \widetilde{Y}=\nu^{-1}(0)$ (que não é necessariamente um divisor em $\widetilde{V}$ ) e seja $\mathcal{Y}=(\nu \circ \widetilde{e})^{-1}(0)$ (divisor em $\left.\mathfrak{X}\right)$. Chamamos de morfismo de Gauss $\gamma$ a restrição à $\widetilde{V}$ da projeção $p r_{2}: V \times G \rightarrow G$. Da mesma forma denotamos $\lambda$ a restrição à $V^{\prime}$ da projeção de $V \times \mathbb{P}^{n}$ sobre $\mathbb{P}^{n}$.

Sejam $\alpha: \Xi \rightarrow \mathbb{P}^{n}$ e $\tau: \Theta \rightarrow G$ os fibrados tautológicos, denotamos:

$$
\begin{aligned}
\xi^{\prime} & =\lambda^{*} \Xi & a^{\prime} & =\lambda^{*} \alpha: \xi^{\prime} \rightarrow V^{\prime} \\
\widetilde{T} & =\gamma^{*} \Theta & \widetilde{t} & =\gamma^{*} \tau: \widetilde{T} \rightarrow \widetilde{V} \\
\xi & =\left(\nu^{\prime}\right)^{*} \xi^{\prime} & a & =\left(\nu^{\prime}\right)^{*} a^{\prime}: \xi \rightarrow \mathfrak{X} \\
T & =(\widetilde{e})^{*} \widetilde{T} & t & =(\widetilde{e})^{*} \widetilde{t}: T \rightarrow \mathfrak{X}
\end{aligned}
$$

e observemos que $\widetilde{t}: \widetilde{T} \rightarrow \widetilde{V}$ é o fibrado de Nash de $V$.

Observemos que $T$ é a restrição à $\mathfrak{X}$ do fibrado $\Theta^{\prime}$ imagem recíproca do fibrado universal $\tau$ : $\Theta \rightarrow G$ pela projeção $V \times \mathbb{P}^{n} \times G \rightarrow G$.

Analogamente, observemos que $\xi$ é a restrição à $\mathfrak{X}$ do fibrado $\Xi^{\prime}$ imagem recíproca do fibrado universal $\alpha: \Xi \rightarrow \mathbb{P}^{n}$ pela projeção $V \times \mathbb{P}^{n} \times G \rightarrow \mathbb{P}^{n}$.

A demonstração do teorema a seguir, devido a Gonzalez-Sprinberg e Verdier, é devida a M. Sebastiani [62].

Teorema 2.9 (G. Gonzalez-Sprinberg e J. L. Verdier):

$$
E u_{V}(0)=\int_{[\mathcal{Y}]} c_{d-1}(T / \xi)
$$

onde $c(T / \xi)=c(T) / c(\xi) \in H^{*}(\mathcal{Y} ; Z)$.

Demonstração: Pela definição de $\mathcal{Y}$, vemos que $\xi$ é a restrição à $\mathfrak{X}$ da imagem recíproca pela projeção $\mathfrak{X} \rightarrow V^{\prime}$. Se $p \in \mathfrak{X}$ e se $x$ é a imagem de $p$ em $V$, então $\xi_{p} \subset \mathbb{C}^{n+1}$ é a reta secante $\overline{0 x}$ se $x \neq 0$; se $x=0$, temos que $\xi_{p} \subset \mathbb{C}^{n+1}$ é uma reta limite. A seção canônica $s$ de $\xi$ é dada por $s(p)=\overline{0 x} \in \xi_{p}$. O conjunto dos seus zeros é naturalmente $\mathcal{Y}$.

Por outro lado, podemos definir uma seção $t$ de $\widetilde{T}$ da seguinte forma, para cada $q \in \widetilde{V}$ definimos $t(q)$ como sendo a projeção ortogonal de $\overline{0 x}$ em $\widetilde{T}_{q} \subset \mathbb{C}^{n}$, onde $x$ é a imagem de $q$ em $V$. Se o 
representante $V$ é suficientemente pequeno, sabemos que $t(q) \neq 0$ para todo $q \in \widetilde{V} \backslash \widetilde{V}_{0}$ (ver [24]). Pela definição e pelo Lema 2.8 temos,

$$
E u_{V}(0)=\left\langle t^{*}(\widetilde{\omega}),[\widetilde{V}]\right\rangle
$$

onde $\widetilde{\omega}$ é a classe de Thom de $\widetilde{T}$.

Seja $t^{\prime}: \mathfrak{X} \rightarrow T$ a seção imagem recíproca de $t$. Então temos,

$$
E u_{V}(0)=\left\langle t^{\prime *}\left(\omega^{\prime}\right),[\mathfrak{X}]\right\rangle
$$

onde $\omega^{\prime}$ é a classe de Thom de $T$, pela funtorialidade da classe de Thom e porque $\mathfrak{X} \rightarrow \widetilde{V}$ induz um homeomorfismo entre abertos densos de $\mathfrak{X}$ e $\widetilde{V}$.

Seja $p \in \mathcal{Y}$ e seja $q$ sua imagem em $\tilde{Y} \subset \widetilde{V}$. Então, existe uma seqüência $\left\{x_{k}\right\}$ de pontos regulares de $V$ tal que $x_{k} \rightarrow 0, \overline{0 x_{k}} \rightarrow \xi_{p}$ e $T_{x_{k}}(V) \rightarrow \widetilde{T}_{q}$, onde $T_{x_{k}}(V)$ é o espaço tangente à $V$ nos pontos regulares $x_{k}$. Pelas propriedades de Whitney, $\xi_{p} \subset T_{p}$. Então, $\xi_{p} \subset T_{p}=\widetilde{T}_{q}$. Logo, se $V$ for suficientemente pequeno, $\xi_{p}$ não é ortogonal à $T_{p}$, para todo $p \in \mathfrak{X}$. Definamos $\phi_{p}: \xi_{p} \rightarrow T_{p}$ pela projeção ortogonal em $T_{p}$.

Notemos que, pela definição de $\phi$ temos $\phi \circ s=t^{\prime}$.

Por outro lado, topologicamente temos $T \cong \xi \oplus(T / \xi)$. Seja $u$ a seção nula de $T / \xi$. Como $\phi \circ s=t^{\prime}$, podemos ver que $t^{\prime}$ corresponde nesta decomposição à $s \oplus u$, então,

$$
t^{\prime *}\left(\omega^{\prime}\right)=s^{*}\left(\omega_{1}\right) \cup u^{*}\left(\omega_{2}\right)
$$

onde $\omega_{1}$ e $\omega_{2}$ são as classes de Thom de $\xi$ e $T / \xi$ respectivamente, assim temos,

$$
\begin{aligned}
& \left\langle t^{* *}\left(\omega^{\prime}\right),[\mathfrak{X}]\right\rangle=\left\langle s^{*}\left(\omega_{1}\right) \cup u^{*}\left(\omega_{2}\right),[\mathfrak{X}]\right\rangle \\
& \left\langle u^{*}\left(\omega_{2}\right), s^{*}\left(\omega_{1}\right) \cap[\mathfrak{X}]\right\rangle=\left\langle u^{*}\left(\omega_{2}\right),[\mathcal{Y}]\right\rangle
\end{aligned}
$$

pela definição de $[\mathcal{Y}]$. Entretanto, $u^{*}\left(\omega_{2}\right)=c_{d-1}\left(T^{\prime} / \xi\right)$, já que o posto de $T / \xi=d-1$ e portanto, $c_{d-1}(T / \xi)$ é a classe de Euler de $T / \xi$. Então temos que,

$$
\left\langle u^{*}\left(\omega_{2}\right),[\mathcal{Y}]\right\rangle=\left\langle c_{d-1}(T / \xi),[\mathcal{Y}]\right\rangle=\int_{[\mathcal{Y}]} c_{d-1}(T / \xi)
$$

\subsection{Obstrução de Euler Local e Multiplicidades Polares}

O cálculo da obstrução de Euler local pela definição original não é fácil. Lê e Teissier [45] mostraram uma fórmula que exprime $E u_{V}(0)$ em função das multiplicidades polares, a qual permite efetuar cálculos de exemplos concretos. Daremos aqui uma idéia da demonstração devida a Lê D. T. e B. Teissier desta fórmula [45].

A notação adotada nesta seção $\operatorname{deg}\left(c_{d-1}(T / \xi) \cap[\mathcal{Y}]\right)$ é apenas outra representação para o valor $\left\langle c_{d-1}(T / \xi),[\mathcal{Y}]\right\rangle=\int_{[\mathcal{Y}]} c_{d-1}(T / \xi)$. 
Teorema 2.10: Seja $(V, 0) \subset\left(\mathbb{C}^{n+1}, 0\right)$ germe de variedade analitica complexa equidimensional de dimensão $d$. Se Vé um representante suficientemente pequeno do germe, e $\mathfrak{D}$ uma bandeira suficientemente genérica em $\mathbb{C}^{n+1}$, temos

$$
(-1)^{d-1} \operatorname{deg}\left(c_{d-1-i}(T) c_{1}(\xi)^{i} \cap[\mathcal{Y}]\right)=m_{0}\left(P_{d-1-i}(\mathfrak{D})\right) .
$$

A demonstração deste resultado encontra-se em [45], e é uma conseqüência de resultados anteriores de [2], [3] [39], [69] e [77].

Teorema 2.11 (Fórmula de Lê-Teissier): Seja $(V, 0) \subset\left(\mathbb{C}^{n+1}, 0\right)$ germe de variedade analítica complexa equidimensinal de dimensão d. Seja $V$ um representante suficientemente pequeno do germe, então temos,

$$
E u_{V}(0)=\sum_{k=0}^{d-1}(-1)^{d-k-1} m_{d-k-1}(V)
$$

onde $m_{i}(V)$ é a multiplicidade da i-variedade polar na origem.

Demonstração: Pela fórmula de Gonzalez-Sprinberg e J. L. Verdier temos que:

$$
E u_{V}(0)=\int_{[\mathcal{Y}]} c_{d-1}(T / \xi)
$$

ou seja, $E u_{V}(0)=\operatorname{deg}\left(c_{d-1}(T / \xi) \cap[\mathcal{Y}]\right)$. Desenvolvendo formalmente $c_{d-1}(T / \xi)$ temos então,

$$
E u_{V}(0)=\sum(-1)^{i} \operatorname{deg}\left(c_{d-1_{i}}(T) c_{1}(/ \xi)^{i} \cap[\mathcal{Y}]\right)
$$

pois $c_{i}(\xi)=c_{1}(\xi)^{i}$, logo pelo Teorema 2.10 segue o resultado.

Exemplo 2.12: Lembremos que no Exemplo 1.48 foram calculadas as multiplicidades polares na origem da variedade singular

$$
V=\left\{(x, y, t) \in \mathbb{R}^{3} ; y^{2}-x^{3}-t^{2} x^{2}=0\right\}
$$

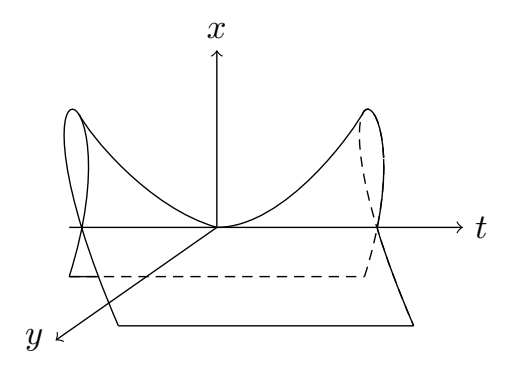

Figura 2.1: Variedade Singular.

Os valores obtidos foram, $m_{0}(V, 0)=2$ e $m_{1}(V, 0)=1$. Usando a fórmula de Lê e Teissier apresentada no Teorema 2.11, obtemos

$$
E u_{V}(0)=2-1=1
$$


Observação 2.13: Como propriedades importantes da obstrução de Euler local temos os seguintes resultados:

- A obstrução de Euler local em um ponto regular é igual à 1. Isto é fácil ver se usamos a definição de obstrução local de Euler por campos radiais.

- A obstrução de Euler local em um ponto de uma curva é exatamente a multiplicidade do ponto sobre a curva [24]. Esta propriedade sairá também diretamente como corolário do teorema 2.15.

- Um outro resultado importante sobre a obstrução de Euler local, mostrado inicialmente por J.-P. Brasselet e M.-H. Schwartz [10], e depois por vários autores, é que a obstrução de Euler local é constante ao longo de cada estrato de uma estratificação Whitney regular. Este resultado será muito importante para a próxima seção, por isso vamos dar uma idéia da sua prova.

Proposição 2.14: Sejam $(V, 0) \subset\left(\mathbb{C}^{n+1}, 0\right)$ um germe de variedade analítica complexa e $\left\{V_{\alpha}\right\}$ uma estratificação de Whitney de $V$. Então, a obstrução de Euler local é constante ao longo de cada estrato da estratificação Whitney $\left\{V_{\alpha}\right\}$.

Resumo da demonstração: Sem perda de generalidade, suponhamos que $V \in\left\{V_{\alpha}\right\}$ seja o estrato contendo a origem e que $V \neq\{0\}$. Seja $p \in V$ um ponto qualquer e $0<\eta<<\varepsilon$. Pela propriedade de equisingularidade sobre um estrato de uma estratificação de Whitney, temos que $\mathbb{B}_{\eta}(p)$ é homotópico à $\mathbb{B}_{\varepsilon}(0)$, logo temos $E u_{V}(p)=E u_{V}(0)$.

\subsection{Uma Fórmula do Tipo Lefschetz}

A fórmula principal provada em [7] é uma fórmula do tipo Lefschetz, para a obstrução de Euler local. Ela é equivalente a dizer que a obstrução de Euler local, como função construtível, satisfaz à condição local de Euler (em teoria bivariante) no que diz respeito às formas lineares genéricas.

Teorema 2.15 ([7], Teo. 3.1): Seja $(V, 0) \subset(M, 0)$ um germe de variedade analitica complexa como acima e $\left\{V_{\alpha}\right\}$ uma estratificação de Whitney de $V$. Seja l $: U \rightarrow \mathbb{C}$ uma forma linear genérica, onde $U$ é uma vizinhança aberta de 0 em $M$. Temos então:

$$
E u_{V}(0)=\sum \chi\left(V_{\alpha} \cap B_{\epsilon} \cap l^{-1}\left(t_{0}\right)\right) \cdot E u_{V}\left(V_{\alpha}\right)
$$

onde $\epsilon$ é suficientemente pequeno, $t_{0} \in \mathbb{C} \backslash\{0\}$ está situado perto da origem e $E u_{V}\left(V_{\alpha}\right)$ é a obstrução de Euler de $V$ em qualquer ponto do estrato $V_{\alpha}$.

A demonstração deste teorema encontra-se em [7], entretanto, o resultado sai diretamente como corolário do resultado principal de [9] que será tratado na próxima seção.

Exemplo 2.16: Consideremos a função $f(x, y, t)=y^{2}-x^{3}-t^{2} x^{2}$ e denotemos $V=f^{-1}(0)$. Consideremos a estratificação definida por $\left\{V_{0}=\{0\}, V_{1}=\{e i x o-t\}, V_{2}=V_{\text {reg }}\right\}$. Esta é uma estratificação de Whitney de $V$. 


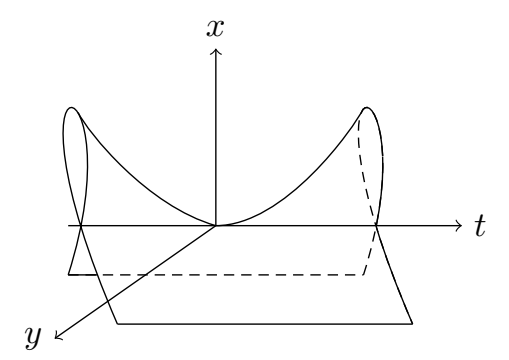

Figura 2.2: Variedade Singular.

Consideremos a forma $l(x, y, t)=t$. A fórmula precedente diz que:

$$
\begin{aligned}
E u_{V}(0) & =\chi\left(V_{0} \cap B_{\epsilon} \cap l^{-1}\left(t_{0}\right)\right) \cdot E u_{V}\left(V_{0}\right) \\
& +\chi\left(V_{1} \cap B_{\epsilon} \cap l^{-1}\left(t_{0}\right)\right) \cdot E u_{V}\left(V_{1}\right) \\
& +\chi\left(V_{2} \cap B_{\epsilon} \cap l^{-1}\left(t_{0}\right)\right) \cdot E u_{V}\left(V_{2}\right) .
\end{aligned}
$$

Mas, como $V_{0} \cap B_{\epsilon} \cap l^{-1}\left(t_{0}\right)=\emptyset$ temos então $\chi\left(V_{0} \cap B_{\epsilon} \cap l^{-1}\left(t_{0}\right)\right)=0$.

Por outro lado $V_{1} \cap B_{\epsilon} \cap l^{-1}\left(t_{0}\right)=\left\{\left(0,0, t_{0}\right)\right\}$, portanto, $\chi\left(V_{1} \cap B_{\epsilon} \cap l^{-1}\left(t_{0}\right)\right)=1$.

Logo $V_{2} \cap B_{\epsilon} \cap l^{-1}\left(t_{0}\right)=\left\{(x, y, t) / y^{2}-x^{3}-t_{0}^{2} x^{2}=0\right\} \backslash\left\{\left(0,0, t_{0}\right)\right\}$.

Com a ajuda do Teorema 2 de [33] podemos calcular $\chi\left(V_{2} \cap B_{\epsilon} \cap l^{-1}\left(t_{0}\right)\right)=-1$

Pelo primeiro ítem da Observação 2.13 temos $E u_{V}\left(V_{2}\right)=E u_{V}\left(V_{\text {reg }}\right)=1$. Agora, se aplicarmos novamente a fórmula para calcular $E u_{V}\left(V_{1}\right)$, usando o também o segundo ítem da Observação 2.13, obtemos $E u_{V}\left(V_{1}\right)=2$. Temos então:

$$
E u_{V}(0)=0 \cdot E u_{V}(0)+1 \cdot 2+(-1) \cdot 1=1 .
$$

Observação 2.17: Consideremos o exemplo de Briançon-Speder, $\phi=x^{5}+y^{7} z+z^{15}+t x y^{6}$, que é uma familia quase-homogênea de hipersuperfícies do tipo $(3,2,1 ; 15)$, com singularidade isolada, portanto topologicamente trivial. Entretanto considerando uma seção genérica $z=a x+b y$, obtemos a família $x^{5}+y^{7}(a x+b y)+(a x+b y)^{15}+t x y^{6}$ de curvas planas. Para calcular o número de Milnor dos elementos desta família, basta calcular o número de Milnor dos termos do tipo $x^{5}+b y^{8}+t x y^{6}$, ou seja, uma família quase-homogênea do tipo $(8,5 ; 40)$. 


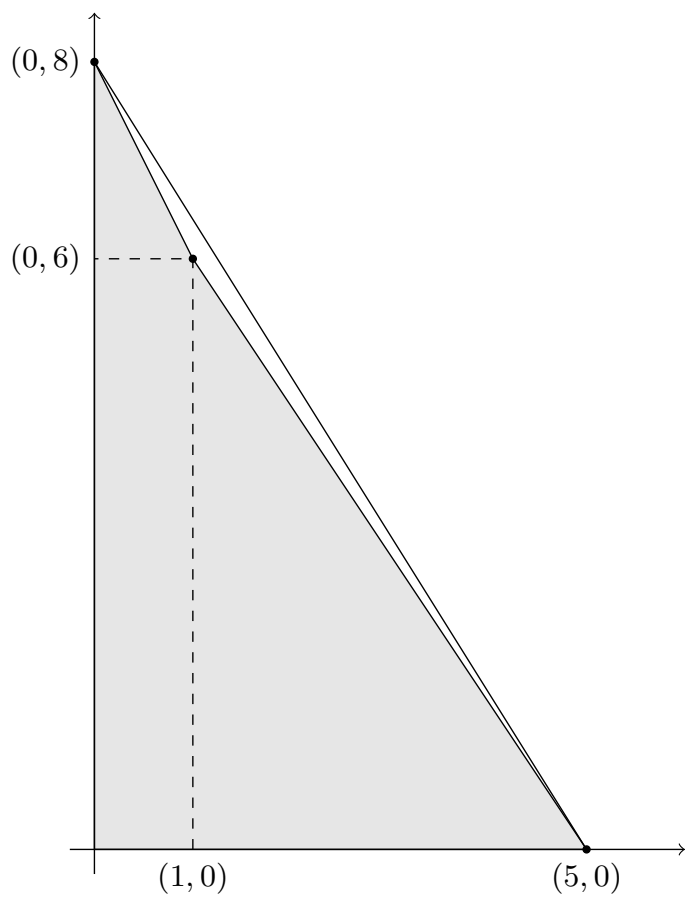

Dos resultados de Lê e Teissier apresentados neste capítulo, segue que $E u_{V_{t}}(0)=m_{0}\left(V_{t}\right)-$ $m_{1}\left(V_{t}\right)$, onde $m_{0}\left(V_{t}\right)$ e $m_{1}\left(V_{t}\right)$ são multiplicidades polares da hipersuperfície $V_{t}$ na origem.

As multiplicidades polares se relacionam diretamente com os números de Milnor de uma variedade [46, 68], através da equação $m_{i}\left(V_{t}\right)=\mu_{i}\left(\phi_{t}\right)+\mu_{i+1}\left(\phi_{t}\right)$ onde $\mu_{i}$ é o número de Milnor da interseção da hipersuperfície com um hiperplano de dimensão $i$. Logo obtemos que $E u_{V_{t}}(0)=$ $m_{0}\left(V_{t}, 0\right)-\mu_{1}\left(\phi_{t}\right)-\mu_{2}\left(\phi_{t}\right)$.

Entretanto sabemos que $m_{0}\left(V_{t}, 0\right)-1=\mu_{1}\left(\phi_{t}\right)$, logo temos $E u_{V_{t}}(0)=1-\mu_{2}\left(\phi_{t}\right)$. Segue portanto, observado o polígono de Newton acima, que $\mu_{2}\left(\phi_{0}\right)<\mu_{2}\left(\phi_{t}\right)$, logo não é constante para a família. Sendo assim, a obstrução de Euler de uma variedade analítica $V$ não é um invariante topológico. 


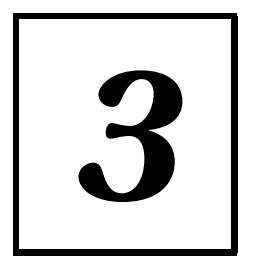

\section{Obstrução de Euler de $f$}

Um invariante importante de um germe de função analítica $f:\left(\mathbb{C}^{n}, 0\right) \rightarrow(\mathbb{C}, 0)$ com ponto crítico isolado na origem é o seu número de Milnor, denotado por $\mu(f)$, definido com sendo $\operatorname{dim}_{\mathbb{C}} \mathcal{O}_{n} / J(f)$, onde $\mathcal{O}_{n}$ é o anel dos germes na origem de funções analíticas e $J(f)$ é o ideal jacobiano de $f$.

Este invariante fornece várias informações sobre a geometria de $f$, por exemplo, no caso em que $f$ tem ponto crítico isolado na origem os seguintes invariantes coincidem a menos de sinal.

(a) O número de Milnor de $f$ em 0 , denotado por $\mu(f)$;

(b) O número de pontos de Morse de uma Morsificação de $f$;

(c) $\mathrm{O}$ índice de Poincaré-Hopf do campo gradiente de $f$ conjugado $\overline{\nabla f}$.

Suponhamos agora que $(V, 0)$ seja um germe de um espaço analítico complexo mergulhado em $\mathbb{C}^{n}$. Uma das possíveis generalizações de $(c)$ é a obstrução de Euler de $f$ na origem, denotada por $E u_{f, V}(0)$.

A obstrução de Euler de uma função $f$ foi introduzida por J.-P. Brasselet, D. Massey, A. J. Parameswaran e J. Seade em [9]. O objetivo dos autores em [9] é entender o que impede a obstrução de Euler local de satisfazer à condição de Euler para funções analíticas com singularidade isolada na origem. Este defeito é chamado obstrução de Euler local de $f$.

Em [60] J. Seade, M. Tibar e A. Verjovsky mostram que a obstrução de Euler de $f$ está intimamente ligada ao número de pontos de Morse de uma Morsificação de $f$, para algumas categorias de germes de variedades singulares.

Os autores também comparam $E u_{f, V}(0)$ com diferentes generalizações do número de Milnor para funções com singularidade isolada sobre espaços singulares, como a noção de número de Milnor introduzida por Lê D. T. em [40], a definição para funções definidas em curvas dada por David Mond e D. van Straten [54] e V. Goryunov [25], e a definição de T. Izawa e T. Suwa [37] para funções definidas em interseções completas em geral.

X. Gómez-Mont, J. Seade and A. Verjovsky introduziram em [23] uma noção de índice chamada de índice GSV, quando $(V, 0)$ é uma interseção completa com singularidade isolada. J. Seade, M. Tibar e A. Verjovsky também em [60] comparam o índice GSV com a $E u_{f, V}(0)$. 
Para o desenvolvimento deste capítulo introduziremos noções básicas sobre funções complexas definidas em espaços singulares, mas especificamente variedades analíticas. Em [41] Lê D. T. introduziu a noção de funções analíticas com singularidade isolada sobre um espaço analítico complexo $V$ com uma estratificação de Whitney $\left\{V_{\alpha}\right\}$.

Neste capítulo apresentamos novos resultados sobre a obstrução de Euler de $f$, relacionando este invariante com a noção de número de Milnor de uma função definida sobre uma variedade algébrica, introduzida por J. W. Bruce e R. M. Roberts em [13], aqui denotado por $\mu_{B R}(f)$.

Para algumas classes de variedades analíticas que incluem os divisores livres, o resultado principal, Teorema 3.61 apresenta uma fórmula útil para o cálculo da obstrução de Euler de $f$, uma vez que o número Milnor $\mu_{B R}(f)$ é um invariante definido como a dimensão de uma álgebra finita.

Como aplicação dos resultados estudamos famílias de funções definidas em hipersuperfícies com singularidade isolada e mostramos que $\mu_{B R}(f)$ é um invariante topológico.

\subsection{Singularidades Isoladas de Funções}

Definição 3.1 ([22]): Sejam $M$ e $P$ variedades analíticas regulares, $V \subset M$ um subconjunto analítico, $\left\{V_{\alpha}\right\}$ uma estratificação de $V$, e $f: M \rightarrow P$. Dizemos que o conjunto $\left(V,\left\{V_{\alpha}\right\}\right)$ é topologicamente trivial sobre $P$ se existe um conjunto estratificado $(F, \mathfrak{F})$ e um homeomorfismo $h: V \approx P \times F$ com $f=p r_{p} \circ h$, levando $\left\{V_{\alpha}\right\}$ na estratificação produto $P \times \mathfrak{F}$ (pr $r_{P}$ denota a projeção sobre $\left.P\right)$.

Dizemos que $\left(V,\left\{V_{\alpha}\right\}\right)$ é localmente trivial sobre $P$ se cada ponto $y \in P$ tem uma vizinhança aberta $U$ tal que $\left(V \cap f^{-1}(U),\left\{V_{\alpha}\right\} \cap f^{-1}(U)\right)$ seja trivial sobre $U$.

O homeomorfismo $h: V \cap f^{-1}(U) \approx V \times F$ é chamado de trivialização local.

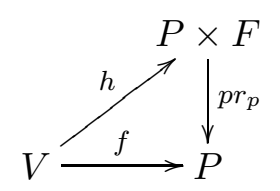

Definição 3.2: Uma aplicação $f: V \rightarrow P$ é uma submersão estratificada se $\left.f\right|_{V_{\alpha}}$ for submersão para cada estrato $V_{\alpha}$ de $V$.

Teorema 3.3 (Primeiro Lema de Isotopia de Thom-Mather): Sejam $M$ e $P$ variedades analíticas regulares, $V$ uma variedade analítica $V \subset M$ e $f: V \rightarrow P$ uma submersão estratificada própria. Então f é uma uma fibração localmente trivial e $\left(V,\left\{V_{\alpha}\right\}\right)$ é localmente trivial sobre $P$.

Como aplicação deste resultado podemos descrever um teorema de Lê D. T. que estende o Teorema de Fibração de Milnor para funções definidas em variedades singulares [40]. Primeiramente precisamos de uma definição para esclarecer o que significa uma função singular definida em um espaço singular.

Definição 3.4: Seja $f: V \rightarrow \mathbb{C}$ uma função analítica complexa, definida sobre um conjunto analítico Whitney estratificado $V$ com estratificação $\left\{V_{\alpha}\right\}$. Dizemos que $f$ tem singularidade isolada em $x \in V$, com respeito à estratificação $\left\{V_{\alpha}\right\}$ se existe $\varepsilon>0$ tal que $\left.f\right|_{B_{\varepsilon}(x) \cap\left(V_{\alpha} \backslash\{x\}\right)} \rightarrow \mathbb{C}$ é submersão para todo $V_{\alpha} \in\left\{V_{\alpha}\right\}$. 
Teorema 3.5 ([40], Teo 1.3): Sejam $V$ uma variedade analítica complexa e $f: V \rightarrow \mathbb{C}$ uma função analítica complexa com singularidade isolada em $x$ com respeito à estratificação de Whitney $\left\{V_{\alpha}\right\}$ de $V$. Então existem números reais positivos $\varepsilon$ e $\eta$ suficientemente pequenos, tais que a aplicação

$$
\phi: B_{\varepsilon}(x) \cap V \cap f^{-1}\left(D_{\eta}^{*}\right) \rightarrow D_{\eta}^{*}
$$

induzida por $f$ é uma fibração localmente trivial sobre o disco menos um ponto

$$
D_{\eta}^{*}=\{y \in \mathbb{C}: 0<|y|<\delta\} .
$$

Demonstração: Seja $U$ uma vizinhança aberta de $x$ em $\mathbb{C}^{n}$, escolhida de tal forma que a restrição de $f$ aos estratos de $U \cap V$ tenha posto 1 exceto possivelmente em $x$. A fibra $U \cap f^{-1}(f(x))$ é estratificada por $\{x\}$ e pelas interseções não vazias dos estratos de $\left\{V_{\alpha}\right\} \operatorname{com} U \cap f^{-1}(f(x))$ menos $\{x\}$. Chamaremos estas estratificações de $U \cap V$ e de $U \cap f^{-1}(f(x))$ de estratificações induzidas por $\left\{V_{\alpha}\right\}$.

Seja $\bar{B}_{\varepsilon}(x)$ a bola fechada em $\mathbb{C}^{n}$ centrada em $x$ com raio $\varepsilon$. Se $\varepsilon>0$ for suficientemente pequeno, digamos $\varepsilon_{0}>\varepsilon>0$, para algum $\varepsilon_{0}$, então o Teorema de Bertini-Sard (Teorema 2.4) diz que a esfera $S_{\varepsilon}(x)$ que limita a bola $B_{\varepsilon}(x)$ intersecta transversalmente os estratos de $\left\{V_{\alpha}\right\}$ e os estratos de $U \cap f^{-1}(f(x))$ induzidos por $\left\{V_{\alpha}\right\}$.

A estratificação de Whitney $\left\{V_{\alpha}\right\}$ de $V$ induz uma estratificação de Whitney sobre $\bar{B}_{\varepsilon}(x) \cap V$ cujos estratos são as interseções não vazias dos estratos de $\left\{V_{\alpha}\right\}$ com a bola aberta $B_{\varepsilon}(x)$ e com a esfera $S_{\varepsilon}(x)$ respectivamente. Pelo teorema de Bertini-Sard, para cada ponto de $S_{\varepsilon}(x) \cap f^{-1}(f(x))$, existe uma vizinhança tal que a restrição de $f$ aos estratos de $S_{\varepsilon}(x) \cap V$ tem posto real 2. Como a interseção $S_{\varepsilon}(x) \cap V$ é compacta, existe um $\eta_{\varepsilon}$ tal que, para qualquer $\eta$ tal que $\eta_{\epsilon}>\eta>0$, a restrição de $f$ aos estratos de $S_{\varepsilon}(x) \cap V$ tem posto 2 para qualquer ponto em $f^{-1}\left(D_{\eta}(f(x))\right.$ ). A aplicação $\bar{\phi}_{\varepsilon, \eta}$ de $\bar{B}_{\varepsilon}(x) \cap V \cap f^{-1}\left(D_{\eta}(f(x))\right)$ sobre o disco $D_{\eta}(f(x))$, induzida pela função $f$ é portanto estratificada (cf. [44]). Como a aplicação $\bar{\phi}_{\varepsilon, \eta}$ é própria, pelo Primeiro Lema de Isotopia de Thom-Mather (3.3), esta aplicação é uma fibração contínua localmente trivial sobre o disco menos um ponto $D_{\eta}^{*}(f(x))$. Por outro lado, a restrição de $f$ aos estratos de $S_{\varepsilon}(x) \cap V \cap f^{-1}\left(D_{\eta}(f(x))\right)$ tem posto máximo, e o Lema de Isotopia de Thom-Mather novamente diz que $f$ induz uma fibração de $S_{\varepsilon}(x) \cap V \cap f^{-1}\left(D_{\eta}(f(x))\right)$ sobre o disco $D_{\eta}(f(x))$. Sobre o disco menos um ponto $D_{\eta}^{*}(f(x))$ ela é uma subfibração de $\bar{\phi}_{\varepsilon, \eta}$, portanto a restrição $\phi_{\varepsilon, \eta}$ de $\bar{\phi}_{\varepsilon, \eta}$ em $B_{\varepsilon}(x) \cap V \cap f^{-1}\left(D_{\eta}(f(x))\right)$ é também uma fibração contínua localmente trivial sobre o disco menos um ponto $D_{\eta}^{*}(f(x))$.

\subsection{Obstrução de Euler de $f$}

Seja $(V, 0) \subset(M, 0)$ um germe de variedade analítica complexa, equidimensional de dimensão complexa $n$ e $(M, 0)$ um germe de variedade complexa regular de dimensão complexa $m$. Denotemos $\left\{V_{\alpha}\right\}$ uma estratificação de Whitney de $M$ compatível com $V$. Podemos supor também que a origem $\{0\}$ é um estrato.

Seja agora $f: V \rightarrow \mathbb{C}$, função analítica com singularidade isolada em 0 e restrição de $\tilde{f}: U \rightarrow \mathbb{C}$, onde $U$ é um subconjunto aberto de $M$ contendo $V$. Seguindo a construção de [9], podemos associar a $f$ um campo estratificado denotado por $\bar{\nabla}_{V} f(z)$. Denotemos por $\bar{\nabla} \tilde{f}(z)$ o campo vetorial gradiente 
conjugado de $\tilde{f}$ sobre um ponto $z \in U$, definido por $\bar{\nabla} \tilde{f}(z)=\left(\frac{\overline{\partial \tilde{f}}}{\partial x_{1}}, \cdots, \frac{\overline{\partial \tilde{f}}}{\partial x_{n}}\right)$, onde a barra significa a conjugação complexa. O núcleo $\operatorname{ker}\left(d \tilde{f}_{z}\right)$ é transversal à $T_{z}\left(V_{\alpha}(z)\right)$ para todo $z \in V \backslash\{0\}$, assim

$$
A n g\left\langle\bar{\nabla} \tilde{f}(z), T_{z}\left(V_{\alpha}(z)\right)\right\rangle<\pi / 2
$$

onde $A n g\langle\cdot, \cdot\rangle$ denota o ângulo entre um vetor e um espaço vetorial. Então a projeção de $\bar{\nabla} \tilde{f}(z)$ sobre $T_{z}\left(V_{\alpha}(z)\right)$, que indicamos por $\zeta_{\alpha}(z)$, não é nula.

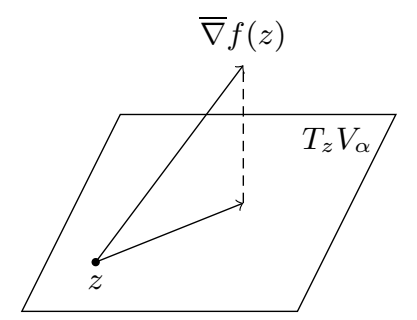

Seja $V_{\beta}$ um estrato tal que $V_{\alpha} \subset \bar{V}_{\beta}$, e seja $\pi: U_{\alpha} \rightarrow V_{\alpha}$ uma vizinhança tubular de $V_{\alpha}$ em $U$. Seguindo a construção de M.-H. Schwartz [59], podemos ver que a condição (a) de Whitney implica que para todo ponto $z \in V_{\beta} \cap U_{\alpha}$, o ângulo entre $\zeta_{\beta}$ e a extensão paralela de $\zeta(\pi(z))$ é pequeno. Esta propriedade implica que estes dois campos de vetores são homotópicos sobre o bordo de $U_{\alpha}$, para $U_{\alpha}$ suficientemente pequeno. Podemos então colar os campos $\zeta_{\alpha}$ para obter um campo estratificado sobre $V$, que denotamos por $\bar{\nabla}_{V} f(z)$. Este campo é homotópico à $\left.\bar{\nabla} \tilde{f}(z)\right|_{V}$ e temos $\bar{\nabla}_{V} f(z) \neq 0$ para todo $z \in V \backslash\{0\}$.

Analogamente ao caso de um campo radial, utilizando a transformada de Nash definida na seção 1.7, podemos também levantar o campo $\bar{\nabla}_{V} f(z)$ sobre $\nu^{-1}\left(V \cap \partial B_{\epsilon}\right)$ sem singularidades. Denotemos este campo por $\tilde{\bar{\nabla}}_{V} f(z)$. O campo $\tilde{\bar{\nabla}}_{V} f(z)$ define um cociclo de obstrução $\operatorname{Obs}\left(\tilde{\bar{\nabla}}_{V} f(z)\right)$, que mede a obstrução para estender $\tilde{\bar{\nabla}}_{V} f(z)$ como seção não-nula de $\widetilde{T}$ sobre $\nu^{-1}\left(V \cap B_{\epsilon}\right)$ :

$$
\operatorname{Obs}\left(\tilde{\bar{\nabla}}_{V} f(z)\right) \in Z^{2 n}\left(\nu^{-1}\left(V \cap B_{\epsilon}\right), \nu^{-1}\left(V \cap \partial B_{\epsilon}\right)\right) .
$$

Desta forma podemos propor a seguinte definição.

Definição 3.6 ([9], Def. 2.1): A obstrução de Euler local de $f$ na origem, denotada por $E u_{f, V}(0)$ é o inteiro obtido pela avaliação de $\operatorname{Obs}\left(\tilde{\bar{\nabla}}_{V} f(z)\right)$ sobre a classe fundamental $\left[\nu^{-1}\left(V \cap B_{\epsilon}\right), \nu^{-1}\left(V \cap \partial B_{\epsilon}\right)\right]$.

Lema 3.7 ([9], Lem. 2.2): O campo vetorial $\bar{\nabla}_{V} f(z)$ é o levantamento, a menos de homotopia, de um campo de vetores em $\mathbb{C}$, via $d \widetilde{f}$.

Demonstração: O vetor gradiente satisfaz,

$$
d \tilde{f}(\bar{\nabla} f(z))=\|\bar{\nabla} f(z)\|^{2} \in \mathbb{R} \backslash\{0\} .
$$

Isto significa que ele é um levantamento, a menos de escalar, de um campo vetorial constante em um pequeno disco $\mathbb{D} \subset \mathbb{C}$.

Observação 3.8: É fácil ver que se 0 é um ponto regular de $V$ e também um ponto regular de $f$, então $E u_{f, V}(0)=0$. Na proposição abaixo provaremos esse resultado em uma situação mais geral. 
Definição 3.9: Seja $(V, 0) \subset(U, 0)$ um germe de variedade analítica em $\mathbb{C}^{n}$, equipado com uma estratificação de Whitney e seja $f:(V, 0) \rightarrow(\mathbb{C}, 0)$ uma função analítica, restrição de uma função analítica $\widetilde{f}:(U, 0) \rightarrow(\mathbb{C}, 0)$. Dizemos que 0 é um ponto geral de $f($ ou que $f:(V, 0) \rightarrow(\mathbb{C}, 0)$ é geral em 0$)$ se o hiperplano ker $(d \widetilde{f}(0))$ é transversal em $\mathbb{C}^{n}$ a todos os espaços tangentes $T_{x_{n}}\left(V_{\alpha}\right)$, para todo $V_{\alpha}$ e toda seqüência $x_{n} \in V_{\alpha}$ convergindo para 0 .

Proposição 3.10 ([9], Prop. 2.4): Seja 0 um ponto geral de $f:(V, 0) \rightarrow(\mathbb{C}, 0)$. Então,

$$
E u_{f, V}(0)=0
$$

Demonstração: Como primeiro passo definamos a aplicação

$$
\widetilde{T} \subset(U \times G(d, n)) \times \mathbb{C}^{n} \stackrel{\widetilde{F}}{\rightarrow} \mathbb{D}_{\eta} \subset \mathbb{C}
$$

por $\widetilde{F}(x, P, y)=d \widetilde{f}_{x}(y)$. Como 0 é um ponto geral de $f$, então $\widetilde{K}=\widetilde{T} \cap \widetilde{F}^{-1}(0)$ é um subfibrado de $\widetilde{T}$ de codimensão (complexa) 1 e $d \widetilde{F}$ leva o complemento ortogonal de $\widetilde{K}$ isomorficamente sobre $T\left(\mathbb{D}_{\eta}\right)$.

Agora, toda $\widetilde{f}$ que define uma singularidade isolada em 0 em $V$, determina um subfibrado $Q$ de $\left.T \mathbb{C}^{n}\right|_{V \backslash\{0\}}$ sempre transversal à $k e r(d \widetilde{f})$, e a restrição de $d \widetilde{f}$ à $Q$ é um isomorfismos entre $Q$ e $T\left(\mathbb{D}_{\eta}\right)$.

Isto implica que cada vetor não-nulo em $\mathbb{D}_{\eta}$ se levanta de forma compatível, a um campo de vetores em $V \backslash\{0\}$ e também como uma seção de $\widetilde{T}_{V}$. O passo final é apenas notar que como no Lema 3.7, o vetor gradiente $\bar{\nabla}_{V} f(z)$ pode ser obtido por um levantamento de tais campos vetoriais.

Proposição 3.11 ([9], Prop. 2.5): Seja $f:(V, 0) \rightarrow(\mathbb{C}, 0)$ analítica em 0 . Então existe um aberto de Zariski $\Omega_{f}$ dentro do espaço das formas lineares em $\mathbb{C}$, tal que para todo $l \in \Omega_{f}$, o ponto 0 é geral para a aplicação $f+\lambda l: V \rightarrow \mathbb{C}$, para todo $\lambda \in \mathbb{C}^{*}$ suficientemente pequeno.

Lema 3.12 ([9], Lem. 3.2): Seja $(V, 0) \subset(M, 0)$ um germe de variedade analítica complexa, equidimensional de dimensão complexa $n$ em $(M, 0)$ germe de variedade complexa regular de dimensão complexa $m$. Seja $f:(V, 0) \rightarrow(\mathbb{C}, 0)$, analítica. Existe um campo vetorial estratificado $w$ sobre $V \cap\left(B_{\epsilon} \backslash \operatorname{Int}\left(B_{\epsilon^{\prime}}\right)\right)$ onde $0<\epsilon^{\prime}<\epsilon$ tal que:

1. w coincide com $\bar{\nabla}_{V} f$ sobre $V \cap \partial B_{\epsilon^{\prime}}(0)$, e sobre $V \cap \partial B_{\epsilon} w$ é um campo radial;

2. $w$ é tangente à $f^{-1}\left(t_{0}\right)$;

3. $w$ tem apenas um número finito de zeros, e todos estão contidos em $f^{-1}\left(t_{0}\right)$;

4. em cada zero a de w o campo w é transversalmente radial ao estrato que contém a.

Teorema 3.13 ([9], Teo. 3.1): Seja $f:(V, 0) \rightarrow(\mathbb{C}, 0)$ com singularidade isolada na origem e $\left\{V_{\alpha}\right\}$ estratificação de Whitney de V. Então,

$$
E u_{V}(0)=\left(\sum \chi\left(V_{\alpha} \cap B_{\epsilon} \cap f^{-1}\left(t_{0}\right)\right) \cdot E u_{V}\left(V_{\alpha}\right)\right)+E u_{f, V}(0)
$$

onde $\epsilon$ é suficientemente pequeno, $t_{0} \in \mathbb{C} \backslash\{0\}$ é situado próximo da origem e $E u_{V}\left(V_{\alpha}\right)$ é a obstrução local de Euler de $V$ em qualquer ponto do estrato $V_{\alpha}$. 
A demonstração deste resultado segue diretamente do Lema 3.12. Fixemos primeiramente algumas notações. Seja $\epsilon$ suficientemente pequeno tal que toda esfera $\mathbb{S}_{\gamma}$ em $U$ centrada em 0 e de raio $\gamma \leq \epsilon$ intercepta transversalmente todos os estratos de $V \backslash\{0\}$. Para cada $t \in \mathbb{C}$, denotemos por $Y_{t}:=f^{-1}(t)$. Seja $\eta>0$ suficientemente pequeno tal que para cada $t$ no disco $\mathbb{D}_{\eta}$ de raio $\eta$ centrado em $0 \in \mathbb{C}$, a hipersuperfície $Y_{t}$ intercepta transversalmente a esfera $\mathbb{S}_{\epsilon}$. Agora, tome $\epsilon^{\prime}$ com $0<\epsilon^{\prime}<\epsilon$, e um ponto $t_{0} \in \mathbb{D}_{\eta}$ tal que $Y_{t_{0}}$ não encontra a esfera $\mathbb{S}_{\epsilon^{\prime}}$. Note que o estrato $V_{\alpha}$ intercepta $Y_{t_{0}}:=f^{-1}\left(t_{0}\right)$ transversalmente e induz uma estratificação de Whitney para este espaço. O resultado segue das propriedades do campo de vetores do Lema 3.12.

Corolário 3.14 ([9], Cor. 3.3): Seja $f:(V, 0) \rightarrow(\mathbb{C}, 0)$ com singularidade isolada na origem. Seja $l$ uma forma linear em $\Omega_{f}$ como na proposição 3.11, e seja $\lambda \in \mathbb{C}^{*}$ tal que $f_{\lambda}:=f+\lambda l$ seja geral na origem. Sejam $M_{f, V}$ e $M_{f_{\lambda}, V}$ as fibras de Milnor de $f$ e $f_{\lambda}$, respectivamente, em $V$. Então,

$$
E u_{f, V}(0)=\sum\left[\chi\left(V_{\alpha} \cap M_{f_{\lambda}, V}\right)-\chi\left(V_{\alpha} \cap M_{f, V}\right)\right] \cdot E u_{V}\left(V_{\alpha}\right)
$$

Observação 3.15 ([9], Obs. 3.4): Se $V=\mathbb{C}^{n}$ e $f:\left(\mathbb{C}^{n}, 0\right) \rightarrow(\mathbb{C}, 0)$ é uma função analítica com singularidade isolada na origem e número de Milnor $\mu$, temos:

$$
E u_{f, V}(0)=(-1)^{n} \mu
$$

Com efeito, a característica de Euler-Poincaré da fibra de Milnor de $f$ é igual à $1+(-1)^{n-1} \mu$ (Ver [52]).

\subsection{Morsificação de funções}

Seja $(V, 0)$ um germe na origem de um espaço analítico complexo, reduzido, equidimensional, mergulhado em $\mathbb{C}^{n}$. Consideremos $\left\{V_{\alpha}\right\}$ uma estratificação de Whitney de um representante $V$ suficientemente pequeno do germe. Como toda estratificação de Whitney é localmente finita, e $V$ um representante suficientemente pequeno do germe, podemos então supor que a estratificação de $V$ tem apenas um número finito de estratos, ou seja, $\alpha \in\{0,1, \cdots, d\}$ para algum $d \in \mathbb{N}$.

Seja como antes $\tilde{f}:\left(\mathbb{C}^{n}, 0\right) \rightarrow(\mathbb{C}, 0)$ uma extensão de $f$ para o espaço ambiente. Para definirmos uma função de Morse estratificada usaremos a Definição 3.9 de ponto geral.

Definição 3.16: Dizemos que $f:(V, 0) \rightarrow(\mathbb{C}, 0)$ é Morse estratificada se as seguintes afirmações são verdadeiras.

(a) Se $0 \in V_{\alpha}$, tal que $\operatorname{dim} V_{\alpha} \geq 1$, a restrição de $f$ ao estrato $V_{\alpha}$ tem um ponto de Morse em 0 .

(b) Se $0 \notin V_{\alpha}$, f é geral em 0 com respeito ao estrato $V_{\alpha}$.

Lema 3.17 ([61],Lemma 4.1): Seja $f$ um germe de função analítica em $(V, 0)$ com uma singularidade de Morse na origem no sentido estratificado. Seja $V_{\alpha}$ o estrato que contém a origem, então a obstrução local de Euler de $f$ na origem é zero se $\operatorname{dim} V_{\alpha}<\operatorname{dim} V$, e $E u_{f, V}(0)=(-1)^{\operatorname{dim}_{\mathbb{C}} V}$ se $V_{\alpha}=V_{\text {reg }}$. 
Proposição 3.18 ([60], Prop. 2.3): Seja $f:(V, 0) \rightarrow(\mathbb{C}, 0)$ uma função analítica com singularidade estratificada na origem. Então

$$
E u_{f, V}(0)=(-1)^{\operatorname{dim}_{\mathbb{C}} V} n_{r e g},
$$

onde $n_{\text {reg }}$ é o número de pontos de Morse em $V_{\text {reg }}$ em uma deformação genérica de $f$.

Demonstração: Consideremos uma pequena deformação analítica Morse estratificada $f_{\lambda}$ de $f$ tal que $f_{\lambda}$ tenha apenas pontos de Morse dentro da bola $\mathbb{B}$.

Como $f_{\lambda}$ é uma deformação de $f$, segue que $\bar{\nabla}_{V} f(z)$ é homotópico à $\bar{\nabla}_{V} f_{\lambda}(z)$ sobre $V \cap \partial \mathbb{B}$, então a obstrução para estender seus levantamentos à $\nu^{-1}(V \cap \mathbb{B})$ sem singularidades é a mesma.

Por outro lado, a obstrução correspondente à $\bar{\nabla}_{V} f_{\lambda}(z)$ é dada pela soma das obstruções obtidas em torno de cada ponto de Morse de $f$. Pelo Lema 3.17 concluímos então que $E u_{f, V}$ é igual à $(-1)^{\operatorname{dim}_{\mathbb{C}} V}$ vezes o número de pontos de Morse de $f_{\lambda}$ sobre $V_{\text {reg }}$.

\subsection{O Link Complexo}

No estudo de estratificações de Whitney analíticas complexas, M. Goresky e R. MacPherson introduziram o conceito de link complexo de um estrato de Whitney e mostraram que o link complexo é o elemento chave para uma teoria de Morse em espaços analíticos complexos [26]. Este conjunto contém várias informações sobre o comportamento de $V$ em torno de $x$.

Definição 3.19: Seja $x$ um ponto de $V \subset \mathbb{C}^{n}$ variedade analítica complexa. Então, para $l: \mathbb{C}^{n} \rightarrow \mathbb{C}$ função linear genérica, o link complexo de $x$ é

$$
\mathcal{L}_{x}=B_{\varepsilon}(x) \cap V \cap l^{-1}(t)
$$

onde $t \neq l(x)$ está suficientemente próximo de $l(x)$.

Teorema 3.20 ([26, 44]): Existe um aberto de Zariski $\Omega$ no espaço das formas lineares complexas de $\mathbb{C}^{n}$ tal que para todo $l \in \Omega$, existe $\varepsilon_{l}>0$ tal que, para qualquer $\varepsilon, \varepsilon_{0}>\varepsilon>0$, existe um $\tau_{\varepsilon, l}$ tal que, para qualquer $t, \tau_{\varepsilon, l}>t>0$ o tipo de homotopia do link complexo

$$
\mathcal{L}_{x}:=B_{\varepsilon}(x) \cap V \cap l^{-1}(l(x)+t)
$$

é um invariante analítico do germe $(V, x)$.

Consideremos $V$ um conjunto Whitney estratificado como anteriormente, e $x$ um ponto de $V$. Seja $V_{\alpha}$ o estrato que contém $x$, agora tomemos $N$ um subespaço afim de $\mathbb{C}^{n}$ de codimensão igual à dimensão do estrato $V_{\alpha}$ e transversal ao estrato, ou seja, tal que $N \cap V_{\alpha}=\{x\}$.

Definição 3.21: O link complexo de $x$ no conjunto $V \cap N$ é chamado de link complexo do estrato $V_{\alpha}$ e é denotado por $\mathcal{L}_{V_{\alpha}}$.

Teorema $3.22([26,44])$ : O tipo de homotopia do link complexo do estrato é um invariante analítico do germe $(V, x)$ e não depende da escolha do $x$ no estrato. 
Definição 3.23: Nas condições apresentadas acima, o espaço $V \cap N \cap B_{\varepsilon}(x)$ é chamado de uma fatia normal $\mathcal{N}_{x}$ em $V$ de $V_{\alpha}$ em $x$, e o par $\left(\mathcal{N}_{x}, \mathcal{L}_{x}\right)$ é chamado de dados normais de Morse do estrato $V_{\alpha}$ em $V$.

\subsection{Profundidade Homotópica Retificada}

Daremos agora uma pequena introdução à noção de Profundidade Homotópica Retificada, denotada por $r h d$. As principais referências para esta seção foram [36] e [40]. A profundidade homotópica retificada foi introduzida por Grothendieck em [28] para medir a falha do Teorema das seções hiperplanas de Lefschetz para espaços singulares. O teorema original diz o seguinte:

Teorema 3.24 (Teorema das seções hiperplanas de Lefschetz): Suponhamos que $V \subset \mathbb{C P}^{m}$ seja uma variedade projetiva não singular e $H$ um hiperplano, então $\pi_{i}(V, V \cap H)=0$ para $i<\operatorname{dim}(V)$.

Nosso interesse na $r h d$ vem do fato que medindo a rhd para espaços analíticos complexos obtemos informações sobre seus dados normais de Morse.

Primeiramente daremos a seguinte definição devida a Grothendieck ([28]).

Definição 3.25: Sejam $(V, 0)$ germe de um espaço analítico complexo, $V$ um representante suficientemente pequeno do germe e $Y$ um subconjunto analítico fechado de $V$. Dizemos que $V$ tem profundidade homotópica $h d_{Y}(V)>n$ ao longo de $Y$ se, para qualquer $y \in Y$, existe um sistema fundamental de vizinhanças $U_{\lambda}$ de y em $V$ tal que os pares $\left(U_{\lambda}, U_{\lambda} \backslash Y\right)$ sejam $(n-1)$-conexos.

O número inteiro $h d_{Y}(V)$ é o máximo do conjunto dos inteiros $n$ como na definição acima.

Definição 3.26: Sejam $V$ como anteriormente, $x$ um ponto de $V$ e $Y \subset V$ um subespaço analítico. Assuma que $x$ esteja em $Y$. Dizemos que o espaço $V$ (ou o germe $(V, x)$ ) tem profundidade homotópica $h d_{Y}(V, x) \geq n$ ao longo de $Y$ no ponto $x$ se existe uma vizinhança aberta $U$ de $x$ em $V$ tal que a profundidade homotópica $h d_{Y \cap U}(V \cap U)$ de $V \cap U$ ao longo de $Y \cap U$ seja maior ou igual à $n$.

Lema 3.27 ([40], Lem. 4.6): Seja $\left\{V_{\alpha}\right\}$ uma estratificação de Whitney de $V$. A função $h d_{V_{\alpha}}(V, x)$ é constante ao longo dos estratos $V_{\alpha}$ de $V$.

Agora podemos definir a profundidade homotópica retificada.

Definição 3.28: Dizemos que a profundidade homotópica retificada $r h d(V, x)$ de $V$ em um ponto $x$ é maior ou igual a n se, para qualquer subespaço analítico fechado $Y$ de $V$, existe uma vizinhança $U$ de $x$ em $V$ tal que a profundidade homotópica de $V \cap U$ ao longo de $Y \cap U$ seja maior do que $n-\operatorname{dim} Y$.

O inteiro $r h d(V, x)$ é o máximo do conjunto de inteiros $n$ como na definição acima.

Teorema 3.29 ([40], Teo. 4.8): Sejam (V,0) germe de variedade analítica complexa (cf. Seção 1.5), $x$ um ponto em $V$ e $\left\{V_{\alpha}\right\}$ uma estratificação de Whitney para $V$. As seguintes condições são equivalentes: 
(a) $\operatorname{rhd}(V, x) \geq n$.

(b) Para qualquer estrato $V_{\alpha}$ de $\left\{V_{\alpha}\right\}$ que contenha o ponto $x$ em seu fecho, o dado normal de Morse $(\mathcal{N}, \mathcal{L})$ de $V_{\alpha}$ em $V$ é $\left(n-\operatorname{dim}\left(V_{\alpha}\right)-1\right)$-conexo.

Obtemos imediatamente então o seguinte corolário.

Corolário 3.30 ([40], Cor. 4.10): Seja $(V, 0)$ germe de variedade analítica complexa e $x$ um ponto em V. Seja $\left\{V_{\alpha}\right\}$ uma estratificação de Whitney de $V$. As seguintes condições são equivalentes:

(a) $\operatorname{rhd}(V, x)=\operatorname{dim}(V, x)$.

(b) Para qualquer estrato $V_{\alpha}$ de $\left\{V_{\alpha}\right\}$ que contenha o ponto $x$ em seu fecho, um link complexo $\mathcal{L}$ de $V_{\alpha}$ em $V$ tem o tipo de homotopia de um buquê de esferas de dimensão real igual à dimensão complexa de $\mathcal{L}$.

De forma similar podemos definir a profundidade homológica retificada $r H d(V, x)$ trocando os grupos de homotopia relativos pelos grupos de homologia relativos.

Em [36] o autor observa a seguinte relação, apresentada aqui de forma simplificada no lema abaixo.

Lema 3.31 ([36], Lema 7.2):

$$
r h d(V, x) \leq r H d(V, x) \leq \operatorname{dim}(V, x)
$$

Para esta noção de profundidade temos o seguinte resultado.

Teorema 3.32 ([40], Teo 4.12): Sejam $V$ como anteriormente, $x$ um ponto em $V$ e $\left\{V_{\alpha}\right\}$ uma estratificação de Whitney para $V$, as seguintes condiçôes são equivalentes:

(a) $\operatorname{rHd}(V, x)=\operatorname{dim}(V, x)$.

(b) Para qualquer estrato $V_{\alpha}$ de $\left\{V_{\alpha}\right\}$ que contenha o ponto $x$ em seu fecho, um link complexo $\mathcal{L}$ de $V_{\alpha}$ em $V$ tem o tipo de homologia de um buquê de esferas de dimensão real igual à dimensão complexa de $\mathcal{L}$.

Apresentaremos agora uma generalização dos resultados de Milnor sobre fibras genéricas de funções com singularidade isolada.

Teorema 3.33 ([40], Teo. 5.4): Sejam $V$ como anteriormente e $x$ um ponto em $V$. Consideremos uma função analítica complexa definida sobre $V$ com singularidade isolada em $x$ no sentido estratificado. Então, se $\operatorname{rhd}(V, x)=\operatorname{dim}(V, x)$, uma fibra genérica de $f$ em uma vizinhança de $x$ tem o tipo de homotopia de um buquê de esferas de dimensão real igual a $\operatorname{dim}(V, x)-1$. 


\subsection{Generalizações do Número de Milnor}

Após a preparação da seção anterior, podemos definir um número de Milnor para funções analíticas, com singularidade isolada no sentido estratificado, definidas sobre variedades analíticas com "boas propriedades".

Definição 3.34: Sejam $V$ espaço analítico tal que $\operatorname{rhd}(V, x)=\operatorname{dim}(V, x)$ e $f$ uma função analítica complexa definida sobre $V$ com singularidade isolada em $x$ no sentido estratificado. Denotemos por $M_{f}$ a fibra de Milnor de $f$, definimos então o número de Milnor de Lê, denotado por $\mu_{L}(f)$ como o posto da homologia $\widetilde{H}_{*}\left(M_{f}\right)$.

A comparação deste número de Milnor com a obstrução de Euler de $f$ foi obtida em [60] usando resultados de M. Tibar [71].

Teorema 3.35 (Teorema do Buquê [71]): Seja $\left\{V_{\alpha}\right\}$ uma estratificação de $V$, representante suficientemente pequeno do germe de variedade analítica complexa $(V, 0)$ e seja $f$ uma função analítica complexa com singularidade isolada no sentido estratificado. Então $M_{f}$, a fibra de Milnor de $f$ tem o mesmo tipo de homotopia do buquê:

$$
M_{f} \simeq \bigvee_{\alpha} \bigvee_{\mu_{\alpha}} S^{k_{\alpha}}\left(\mathcal{L}_{V_{\alpha}}\right)
$$

onde $\mathcal{L}_{V_{\alpha}}$ é o link complexo do estrato $V_{\alpha}$, onde denotamos por $k_{\alpha}$ a dimensão complexa de $V_{\alpha}$ e por $S^{k_{\alpha}}\left(\mathcal{L}_{V_{\alpha}}\right)$ a suspensão de $\mathcal{L}_{V_{\alpha}}$ repetida $k_{\alpha}$-vezes. E mais ainda, os números $\mu_{\alpha}$ dependem apenas da estratificação e da própria $f$.

Dos resultados de [71] obtemos que

$$
\widetilde{H}_{*}\left(M_{f}\right)=\tilde{H}_{*}\left(M_{l}\right) \bigoplus \oplus_{\alpha} \widetilde{H}_{*-k_{\alpha}+1}\left(C\left(\mathcal{L}_{V_{\alpha}}\right), \mathcal{L}_{V_{\alpha}}\right),
$$

onde $C\left(\mathcal{L}_{V_{\alpha}}\right)$ denota o cone sobre $\mathcal{L}_{V_{\alpha}}$.

Como foi observado no início do capítulo, no caso regular o número de Milnor e a obstrução de Euler de $f$ estão intimamente ligados. Na comparação destes dois invariantes definidos em variedades singulares, J. Seade, M. Tibar e A. Verjovsky em [60] obtiveram os seguintes resultados:

Teorema 3.36 ([60], Seção 3.1 ): Sejam $V$ um espaço analítico complexo e $0 \in V$. Consideremos uma função analítica complexa definida sobre $V$ com singularidade isolada em 0 no sentido estratificado. Então, se $r H d(V, x)=\operatorname{dim}(V, x)$ temos que

$$
\mu_{L}(f) \geq(-1)^{\operatorname{dim} V}\left(E u_{f, V}(0)\right) .
$$

E mais especificamente, para o caso de interseções completas com singularidade isolada:

Teorema 3.37 ([60], Seção 3.1 ): Sejam $V$ um espaço analítico complexo e $0 \in V$. Consideremos uma função analítica complexa $f$ definida sobre $V$ com singularidade isolada em 0 no sentido estratificado e l uma forma linear genérica. Então, se $V$ for uma ICIS temos que

$$
E u_{f, V}(0)=(-1)^{\operatorname{dim} V}\left[\mu_{L}(f)-\mu_{L}(l)\right] .
$$


Observação 3.38: A definição do número de Milnor devida a V. Goryunov, D. Mond e D. van Straten, que denotamos aqui por $\mu_{G}$, foi originalmente estabelecida para funçôes definidas em curvas singulares (para uma definição precisa ver [54]). Este número de Milnor é invariante por deformaçôes tanto da função como também do espaço em que a função está definida.

No caso em que $f$ está definida sobre uma curva $V$ que é uma interseção completa com singularidade isolada, (denotada aqui por ICIS [46]), é possivel mostrar que $\mu_{G}$ coincide com o índice-GSV do campo de vetores gradiente de $f$ sobre $V$. O indice-GSV de um campo de vetores $v$ sobre um germe de variedade $(V, 0)$ definido em [23] é igual ao indice de Poincaré-Hopf de uma extensão de $v$ sobre a fibra de Milnor $X_{t}$.

Desta forma podemos definir $\mu_{G}$ para as demais dimensões para o caso de ICIS.

No caso em que $V$ é uma ICIS, a relação entre $\mu_{L}$ e $\mu_{G}$ é dada pela equação

$$
\mu_{G}=\mu_{L}+\mu(V, 0)
$$

onde $\mu(V, 0)$ denota o número de Milnor da ICIS $V$ ([46], [60]).

Um resultado de [60] que decorre diretamente do Teorema (3.37) e da observação anterior é:

Teorema 3.39: Sejam $V$ um espaço analítico complexo $e 0 \in V$. Consideremos uma função analítica complexa $f$ definida sobre $V$ com singularidade isolada em 0 no sentido estratificado e l uma forma linear genérica. Então, se V for uma ICIS temos que

$$
E u_{f, V}(0)=(-1)^{\operatorname{dim} V}\left[\mu_{G}(f)-\mu_{G}(l)\right] .
$$

Detalharemos abaixo um exemplo originalmente apresentado em [60], observando inicialmente a seguinte relação dada no Corolário 3.14:

$$
E u_{f, V}(0)=\sum_{i}\left[\chi\left(V_{\alpha} \cap M_{l, X}\right)-\chi\left(V_{i} \cap M_{f, V}\right)\right] \cdot E u_{V}\left(V_{\alpha}\right),
$$

onde $M(f, X)$ e $M(l, X)$ denotam representantes das fibras de Milnor de $f$ e da função linear genérica $l$, respectivamente. Combinando essas relações com a proposição 3.18 , obtemos:

$$
\left.\sum_{i}\left[\chi\left(V_{i} \cap M_{l, V}\right)-\chi\left(V_{i} \cap M_{f, V}\right)\right] \cdot E u_{V}\left(V_{\alpha}\right)\right)=(-1)^{\operatorname{dim} \mathbb{C} V} n_{r e g},
$$

onde $n_{r e g}$ é o número de pontos de Morse sobre $V_{r e g}$ de uma Morsificação de de $f$.

Exemplo 3.40 ([60], Ex 4.1): Seja $V=\left\{x^{2}-y^{2}=0\right\} \times \mathbb{C} \subset \mathbb{C}^{3}$ e considere $f$ a restrição a $V$ da função $(x, y, z) \mapsto x+2 y+z^{2}$. Tome $x_{0}:=(0,0,0)$. Então $V$ possui dois estratos: $W_{0}=$ eixo $-z \mathrm{e}$ $W_{1}=V \backslash\{x=y=0\}$. Tome como função linear geral $l$ a restrição a $V$ da projeção $(x, y, z) \mapsto z$. Calculando $E u_{f, V}(0)$ pela relação 3.1 , podemos ver que $M\left(l, x_{0}\right) \cap W_{0}$ é um ponto e que $M\left(f, x_{0}\right) \cap W_{0}$ é constituído de dois pontos. Além disso, $M\left(l, x_{0}\right) \cap W_{1}$ é a união disjunta de duas cópias de $\mathbb{C}^{*}$ e $M\left(f, x_{0}\right) \cap W_{1}$ é a união disjunta de duas cópias de $\mathbb{C}^{* *}$, onde $\mathbb{C}^{*}$ é $\mathbb{C}$ menos um ponto e $\mathbb{C}^{* *}$ é $\mathbb{C}$ menos dois pontos. Assim, a fórmula 3.1 fornece:

$$
E u_{f, V}(0)=(1-2) \cdot E u_{V}\left(x_{0}\right)+(0-(-2)) \cdot 1 .
$$


Temos que $E u_{V}\left(x_{0}\right)=E u_{V \cap\{l=0\}}\left(x_{0}\right)$. Mas $E u_{V \cap\{l=0\}}\left(x_{0}\right)$ é apenas a característica de Euler do link complexo da fatia $V \cap\{l=0\}=\left\{x^{2}-y^{2}=0\right\}$. Este link complexo é constituído de dois pontos e portanto $E u_{V \cap\{l=0\}}\left(x_{0}\right)=2$.

Logo, obtemos $E u_{f, V}\left(x_{0}\right)=0$.

\subsection{Número de Milnor de Bruce e Roberts}

Bruce e Roberts deram em [13] uma generalização diferente para o número de Milnor de uma função analítica complexa definida sobre um espaço singular. Para trabalhar com essa definição precisaremos de algumas definições.

Seja $\mathcal{I}(V)$ o ideal de $\mathcal{O}_{n}$ formado pelos germes de funções que se anulam em $V$.

Um dos principais objetivos em [13] é caracterizar as classes de equivalência de germes de funções pela relação de equivalência de germes de difeomorfismos que preservam um germe de subvariedade $V$. A técnica usual para isto é a integração de germes de campos de vetores tangentes a $V$. Passamos a descrever tais campos:

Definição 3.41: Seja Der $\mathbb{C}^{n}$ o $\mathcal{O}_{n}$-módulo formado pelos germes na origem de campos de vetores analíticos em $\mathbb{C}^{n}$.

Um campo $\xi \in$ Der $_{0} \mathbb{C}^{n}$ é logarítmico para $V$ se, quando visto como derivação i.e., $\xi=\sum_{j=1}^{n} \xi_{j} \frac{\partial}{\partial x_{j}}$, ocorrer $\xi(h) \in \mathcal{I}(V)$, para qualquer $h \in \mathcal{I}(V)$.

$O \mathcal{O}_{n}$-submódulo formado pelos germes de campos de vetores logarítmicos é denotado por $\Theta_{(V, 0)}$. Quando o ponto $x=0$ estiver subentendido escrevemos apenas $\Theta_{V}$.

Como nos casos anteriores, precisaremos trabalhar aqui com uma estratificação do nosso espaço. Introduziremos aqui a noção de estratificação logarítmica de um espaço analítico.

Lema 3.42 ([13], Lemma 1.5): Seja $V$ representante suficientemente pequeno do germe $(V, 0)$ como acima e $U$ vizinhança aberta da origem. Existe uma única estratificação $\left\{V_{\alpha}\right\}$ de $U$ com as seguintes propriedades:

(i) Cada estrato $V_{\alpha}$ é uma subvariedade suave conexa mergulhada em $U$ e $U$ é a união disjunta $\cup V_{\alpha}$;

(ii) se $x \in V_{\alpha}$ então o espaço tangente $T_{x} V_{\alpha}$ coincide com $\Theta_{V}(x)$;

(iii) se $V_{\alpha}$ e $V_{\beta}$ são dois estratos distintos com $V_{\alpha}$ encontrando o fecho de $V_{\beta}$, então $V_{\alpha}$ está contido na fronteira, $\partial V_{\beta}$, de $V_{\beta}$.

Definição 3.43: A estratificação $\left\{V_{\alpha}\right\}$ como acima é chamada estratificação logarítmica de $V$ e um estrato $V_{\alpha}$ é chamado de estrato logarítmico.

Definição 3.44: O germe $(V, 0)$ é holonômico se para alguma vizinhança $U$ de 0 em $\mathbb{C}^{n}$ a estratificação logarítmica de $U$ tem apenas um número finito de estratos. 
Uma observação importante é que para germes $(V, 0)$ de variedades holonômicas, a estratificação logarítmica de uma vizinhança suficientemente pequena da origem é Whitney regular ([13], Proposição 1.10).

Seja $f:\left(\mathbb{C}^{n}, 0\right) \rightarrow(\mathbb{C}, 0)$ e $J_{V}(f)$ o ideal $\left\{\delta f: \delta \in \Theta_{V, 0}\right\}$ em $\mathcal{O}_{n, 0}$.

Definição 3.45: A multiplicidade de $f$ sobre $V$ em 0 , denotada por $\mu_{B R}(f)$ é definida por $\operatorname{dim}_{\mathbb{C}} \mathcal{O}_{n} / J_{V}(f)$.

O seguinte resultado se verifica:

Proposição 3.46: [17] O germe $f$ tem singularidade isolada em $V$ na origem $\Leftrightarrow \mu_{B R}(f)<\infty$.

Exemplo 3.47: Seja $V:=\left\{(x, y) \in \mathbb{C}^{2} ; x^{3}-y^{2}=0\right\}$ uma variedade analítica de $\mathbb{C}^{2}$. Por [13] sabemos que os seus campos de vetores tangentes são gerados por

$$
\xi_{1}(x, y)=2 x \frac{\partial}{\partial x}+3 y \frac{\partial}{\partial y} \text { e } \xi_{2}(x, y)=2 y \frac{\partial}{\partial x}+3 x^{2} \frac{\partial}{\partial y} .
$$

Sejam,

$$
f(x, y)=y^{3}+x y^{2} \text { e } g(x, y)=3 x^{3}+x y+4 y^{2}
$$

assim,

$$
\begin{aligned}
& J_{V}(f)=\left\langle 8 x y^{2}+9 y^{3}, 6 x^{3} y+9 x^{2} y^{2}+2 y^{3}\right\rangle, \\
& J_{V}(g)=\left\langle 18 x^{3}+5 x y+24 y^{2}, 3 x^{3}+42 x^{2} y+2 y^{2}\right\rangle .
\end{aligned}
$$

Note que a variedade $\mathcal{V}\left(J_{V}(f)\right)$ contém o conjunto $\{(x, 0): x \in \mathbb{C}\}$, sendo assim $\operatorname{dim}_{\mathbb{C}} \mathcal{O}_{2} / J_{V}(f)$ não é um número finito, o fato implícito é que $f$ não corta transversalmente os estratos de $V$ fora da origem.

No caso da função $g$ temos que a variedade $\mathcal{V}\left(J_{V}(g)\right)$ se reduz apenas ao conjunto $\{(0,0)\}$, e temos

$$
\begin{aligned}
J_{V}(g) & =\left\langle 18 x^{3}+5 x y+24 y^{2}, 3 x^{3}+42 x^{2} y+2 y^{2}\right\rangle \\
& =\left\langle 5 x y+12 y^{2}-252 x^{2} y,-5 x y+18 x^{3}+504 x^{2} y\right\rangle \\
& =\left\langle y\left(5 x+12 y-252 x^{2}\right), x\left(-5 y+18 x^{2}+504 x y\right)\right\rangle .
\end{aligned}
$$

Por propriedades de teoria de interseção de curvas, se denotarmos

$$
I=\left\langle 5 x+12 y-252 x^{2},-5 y+18 x^{2}+504 x y\right\rangle
$$

temos que:

$$
\operatorname{dim}_{\mathbb{C}} \mathcal{O}_{2} / J_{V}(g)=\operatorname{dim}_{\mathbb{C}} \mathcal{O}_{2} /\langle y, x\rangle+\operatorname{dim}_{\mathbb{C}} \mathcal{O}_{2} /\left\langle y, x^{2}\right\rangle+\operatorname{dim}_{\mathbb{C}} \mathcal{O}_{2} /\langle y, x\rangle+\operatorname{dim}_{\mathbb{C}} \mathcal{O}_{2} / I
$$

Mas, simplificando temos que

$$
I=\left\langle 25 x-1044 x^{2}+9072 x y, 5 y-18 x^{2}-504 x y\right\rangle,
$$

$\log 0$

$$
\mu_{B R}(g)=1+2+1+1=5
$$




\section{A Variedade Logarítmica Característica}

A variedade logarítmica característica associada à $V$, denotada $L C(V)$ é um elemento de suma importância para os resultados de [13]. A partir das informações obtidas em [13] através desta variedade, vamos obter o resultado principal deste capítulo.

Definição 3.48: Suponhamos que os campos de vetores $\delta_{1}, \ldots, \delta_{m}$ gerem $\Theta_{V}$ em alguma vizinhança $U$ de $0 \in \mathbb{C}^{n}$. Então se $T_{U}^{*} \mathbb{C}^{n}$ é a restrição do fibrado cotangente de $\mathbb{C}^{n}$ em $U$, definimos $L C(U)$ como sendo $\left\{(x, \xi) \in T_{U}^{*} \mathbb{C}^{n}: \xi\left(\delta_{i}(x)\right)=0, i=1 \ldots, m\right\}$.

$L C(V)$ é o germe de $L C_{U}(V)$ em $T_{0}^{*} \mathbb{C}^{n}$, e independe da escolha dos campos de vetores $\delta_{i}$.

Dado um estrato logarítmico $V_{\alpha}$, seja $T^{*} V_{\alpha}$ o espaço conormal de $V_{\alpha}$. Desta forma temos que,

$$
L C(V)=\bigcup_{\alpha} \overline{T^{*} V_{\alpha}}
$$

Proposição 3.49 ([13], Prop. 1.14 (ii)): Seja $V$ representante do germe $(V, 0)$ um espaço holonômico com estratos $\left\{V_{\alpha}\right\}$. Então os conjuntos $\overline{T^{*} V_{\alpha}}$ são as componentes irredutiveis de $L C(V)$.

Exemplo 3.50 ([13], Ex. 1.12): Seja $(V, 0)$ germe da variedade definida por quatro planos passando pela origem da seguinte forma $(x, y, z) \in \mathbb{C}^{3}: x y\left(x^{2}-y^{2}\right)=0$. A estratificação logarítmica é dada pelo eixo- $z$, as componentes do seu complemento dentro da união dos quatro planos, e o complementar dos quatro planos.

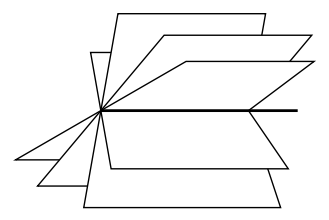

Por [13] sabemos que $\Theta_{V, 0}$ é gerado pelos campos vetoriais:

$$
\left\{\begin{array}{r}
x \partial_{x}+y \partial_{y} \\
\left(x^{2}-y^{2}\right) x \partial_{x}-\left(x^{2}-y^{2}\right) y \partial_{y} \\
\partial_{z}
\end{array}\right.
$$

Sejam $x, y, z, a, b, c$ as coordenadas de $T^{*} \mathbb{C}^{3}$, temos então que $L C(V)$ é a variedade definida por

$$
\left\{\begin{aligned}
x a+y b & =0 \\
\left(x^{2}-y^{2}\right)(x a-y b) & =0 \\
c & =0
\end{aligned}\right.
$$

Esta variedade tem seis componentes irredutíveis, todos 3-planos, correspondendo aos seis estratos logarítmicos de $V$. Cada componente irredutível aparece com multiplicidade 1, exceto pelo fecho do fibrado conormal do estrato logarítmico $x=0=y$, o qual aparece com multiplicidade 3 .

Antes de enunciar o próximo teorema introduziremos algumas definições, para maiores detalhes consultar [77]. 
Definição 3.51: Sejam $A$ um anel e $P_{0}, \cdots, P_{n}$ ideais primos de $A$ tais que $P_{0} \varsubsetneqq P_{1} \varsubsetneqq \cdots \varsubsetneqq P_{n}$, então estes ideais primos formam uma cadeia de ideais primos de altura n. A dimensão de Krull de A é definida como o supremo das alturas de todas as cadeias de ideais primos possiveis de A.

Definição 3.52: Sejam A um anel comutativo e $M$ um A-módulo. Uma seqüência A regular sobre $M$ é uma d-upla de elementos não-nulos e não-unidades $a_{1}, \cdots, a_{d}$ de $A$, tais que para cada $i, o$ elemento $a_{i}$ não seja um divisor de zero no A-módulo quociente $M /\left(a_{1}, \cdots, a_{n}\right) M, 1 \leq i \leq d$.

Definição 3.53: A profundidade de A é definida como a altura máxima de uma seqüência $A$ regular sobre $A$.

Definição 3.54: Um anel local é Cohen-Macaulay se for um anel local noetheriano comutativo com dimensão de Krull igual à sua profundidade.

Definição 3.55: Dizemos que uma variedade analítica é Cohen-Macaulay se seu anel local for Cohen-Macaulay.

Podemos então enunciar o seguinte resultado:

Teorema 3.56 ([13], Cor. 5.8): Seja $(V, 0)$ um germe de variedade analitica complexa, holonômica, $\left\{V_{\alpha}\right\}$ a estratificação logarítmica de um representante suficientemente pequeno de $(V, 0)$, de tal forma que esta estratificação seja Whitney regular. Sejam $f:(V, 0) \rightarrow \mathbb{C}$ uma função analítica complexa com singularidade isolada na origem no sentido estratificado, $n_{\alpha}$ o número de pontos críticos de uma Morsificação estratificada de $f$ no estrato $V_{\alpha}$ e $m_{\alpha}$ as multiplicidades das componentes irredutiveis $\overline{T^{*} V_{\alpha}}$ de $L C(V)$. Então temos que,

$$
\sum_{\alpha} m_{\alpha} n_{\alpha} \leq \operatorname{dim}_{\mathbb{C}} \mathcal{O}_{n} / J_{V}(f)
$$

A igualdade ocorre se, e somente se a variedade $L C(V)$ for Cohen-Macaulay em $(0, d f(0))$.

Apresentaremos agora um exemplo que mostra a importância da condição sobre a variedade $L C(V)$ ser Cohen-Macaulay.

Exemplo 3.57 ([13], Ex. 5.9): Seja $V \subset \mathbb{C}^{n}$ uma variedade regular contendo a origem, podemos assumir que $V$ é definida por $x_{1}=x_{2}=\cdots=x_{l}=0$ para algum $l$ tal que $0<l \leq n$. O módulo dos campos de vetores tangentes a $V$ é gerado por,

$$
\left\{\begin{array}{rl}
x_{i} \partial_{x_{j}}, & i=1, \cdots, l \\
\partial_{x_{i}}, & i=l+1, \cdots, n,
\end{array} \quad j=1, \cdots, l\right.
$$

e a estratificação logarítmica de $\mathbb{C}^{n}$ é $V_{0}=\mathbb{C}^{n} \backslash V, V_{1}=V$.

Se $\left\{x_{1}, \cdots, x_{n}, p_{1}, \cdots, p_{n}\right\}$ são as coordenadas de $T^{*} \mathbb{C}^{n}$, a variedade logarítmica $L C(V)$ é definida por,

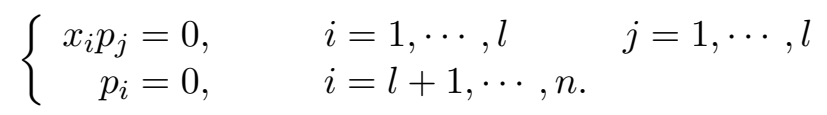


Portanto $L C(V)$ consiste de dois $n$-planos que se intersectam ao longo de um espaço $(n-$ l)-dimensional. Este conjunto é Cohen-Macaulay se, e somente se $l=1$, ou seja, se $V$ é uma hipersuperfície. Se $f\left(x_{1}, \ldots, x_{n}\right)=x_{1}^{2}+x_{2}^{2}+\cdots+x_{n}^{2}$, então $\operatorname{dim}_{\mathbb{C}} \mathcal{O}_{n} / J_{V}(f)=l+1$, mas uma Morsificação de $f$ tem apenas dois pontos críticos, assim vemos que a condição da variedade $L C(V)$ ser Cohen-Macaulay é realmente importante.

Definição 3.58: Um germe de hipersuperfície reduzida $(V, 0) \subset\left(\mathbb{C}^{n}, 0\right)$ é um divisor livre se $\Theta_{V, 0}$ for um $\mathcal{O}_{n}$-módulo livre.

Observação 3.59: Se $V \subset \mathbb{C}^{n}$ for um divisor livre então $\Theta_{V, 0}$ é necessariamente gerado por $n$ elementos.

Teorema 3.60 ( [13], Prop. 6.3): Seja $(V, 0) \subset\left(\mathbb{C}^{n}, 0\right)$ uma variedade analítica reduzida, então quaisquer duas das afirmaçôes abaixo implica a terceira.

(i) A estratificação logarítmica de $V$ é holonômica;

(ii) $L C(V)$ é uma interseção completa;

(iii) $(V, 0)$ é um divisor livre.

Em particular quando $V$ é holonômico e um divisor livre, $L C(V)$ é uma interseção completa, e portanto Cohen-Macaulay. Neste caso temos então que,

$$
\mu_{B R}(h)=\sum_{i=0}^{d} m_{i} n_{i},
$$

onde $n_{i}$ denota o número de pontos de Morse de $f_{t}$ sobre $V_{i}[13]$.

Podemos agora enunciar o principal resultado deste capítulo, que estabelece uma relação entre o número de Milnor de Bruce e Roberts e a obstrução de Euler de $f$.

Teorema 3.61: Sejam $(V, 0)$ germe de variedade analítica reduzida, equidimensional e $f:\left(\mathbb{C}^{n}, 0\right) \rightarrow$ $(\mathbb{C}, 0)$ com singularidade isolada na origem e tal que $f$ tenha singularidade isolada na origem também no sentido estratificado. Se $L C(V)$ for Cohen-Macaulay temos,

$$
\mu_{B R}(f)=\sum_{\alpha=0}^{d} m_{\alpha}(-1)^{\operatorname{dim}_{\mathbb{C}} V_{\alpha}} E u_{f, \bar{V}_{\alpha}}(0),
$$

onde $m_{\alpha}$ denota a multiplicidade de $T^{*} V_{\alpha}$ em $L C(V)$ e $n_{\alpha}$ o número de pontos de Morse de uma Morsificação de $f$ sobre o estrato $V_{\alpha}$.

Demonstração: Como estamos supondo que $L C(V)$ é uma variedade Cohen-Macaulay, temos que

$$
\mu_{B R}(f)=\sum_{\alpha=0}^{d} m_{\alpha} n_{\alpha}
$$

onde $m_{\alpha}$ denota a multiplicidade de $T^{*} V_{\alpha}$ em $L C(V)$ e $n_{\alpha}$ o número de pontos de Morse de uma Morsificação de $f$ sobre o estrato $V_{\alpha}$. 
Dada uma Morsificação $f_{M}$ de $f$, podemos supor que $f_{M}$ restrita à $\bar{V}_{\alpha}$ tem seus pontos de Morse sobre $V_{\alpha}$. Como $n_{\alpha}=(-1)^{\operatorname{dim}_{\mathbb{C}} V_{\alpha}} E u_{f, \bar{V}_{\alpha}}(0)$ (ver [60]), temos então,

$$
\mu_{B R}(f)=\sum_{\alpha=0}^{d} m_{\alpha}(-1)^{\operatorname{dim}_{\mathbb{C}} V_{\alpha}} E u_{f, \bar{V}_{\alpha}}(0) .
$$

Uma classe importante de exemplos onde podemos aplicar esse resultado é quando a variedade $V$ é o discriminante de um germe de aplicação analítica estável $F:\left(\mathbb{C}^{n}, 0\right) \rightarrow\left(\mathbb{C}^{p}, 0\right), n \geq p$, com $(n, p)$ nas boas dimensões de Mather. Neste caso $V$ é um divisor livre e holonômico, logo por [13] temos que $L C(V)$ é Cohen-Macaulay.

Exemplo 3.62: Consideremos $V=\left\{(x, y, z) \in \mathbb{C}^{3} ; x^{2}+y^{2}+z^{3}=0\right\}$, que é uma interseção completa quase-homogênea do tipo $(3,3,2 ; 6)$, mais ainda, um divisor livre.

Os geradores de $\Theta_{V}$ são:

$$
3 x \partial_{x}+3 y \partial_{y}+2 z \partial_{z}, \quad 2 y \partial_{x}-2 x \partial_{y}, \quad 3 z^{2} \partial_{x}-2 x \partial_{z}, \quad 3 z^{2} \partial_{y}-2 y \partial_{z}
$$

Tomemos $f(x, y, z)=z$, projeção no eixo- $z$, que é uma projeção genérica em relação à $V$. É fácil ver que,

$$
J_{V}(f)=\langle 2 z,-2 x,-2 y\rangle=\langle x, y, z\rangle,
$$

$\log 0 \mu_{B R}(f)=1$.

Vamos usar as relações entre os invariantes para calcular as multiplicidades de $L C(V)$, que neste caso, é uma interseção completa.

Temos:

$$
\mu_{B R}(f)=m_{0} \mu(f)+\sum_{\alpha=1}^{d} m_{\alpha}(-1)^{\operatorname{dim}_{\mathbb{C}} V_{\alpha}} E u_{f, \bar{V}_{\alpha}}(0) .
$$

Uma vizinhança $U$ da origem pode ser estratificada por $V_{0}=U \backslash V, V_{1}=V_{\text {reg }}$ e $V_{2}=\{0\}$, e como $\overline{T^{*}(U \backslash V)}=T^{*} U$, temos $m_{0}=1$. Logo

$$
\mu_{B R}(f)-\mu(f)=m_{1}(-1)^{\operatorname{dim}_{\mathbb{C}} V_{1}} E u_{f, \bar{V}_{1}}(0)+m_{2}(-1)^{\operatorname{dim}_{\mathbb{C}} V_{2}} E u_{f, \bar{V}_{2}}(0)
$$

Como $f$ é uma projeção genérica temos $\mu(f)=0$ e mais ainda, $E u_{f, \bar{V}_{1}}(0)=0$. Além disso $E u_{f, F_{2}}(0)=n_{2}$, e portanto $\mu_{B R}(f)=m_{2} n_{2}$. Mas $V_{2}$ contém apenas um ponto, portanto $n_{2}=1$, e $\operatorname{assim} \mu_{B R}(f)=m_{2}$, ou seja, $m_{2}=1$.

Como foi observado em [54], o número de Milnor de Bruce e Roberts tem a vantagem de ser mais facilmente calculado, entretanto pode não se comportar bem em relação a deformações da variedade $V$, já que campos de vetores tangentes não se levantam automaticamente sobre deformações da variedade. Entretanto, aplicaremos o Teorema 3.61 para provar que o número de Milnor de Bruce e Roberts é um invariante topológico para famílias de funções com singularidade isolada definidas em hipersuperfícies com singularidade isolada $V$. Neste caso sabemos que $L C(V)$ é uma variedade Cohen-Macaulay (Teo 3.60). Para isso introduziremos alguns novos conceitos. 
Definição 3.63: Dizemos que $F:\left(\mathbb{C}^{n} \times \mathbb{C}^{r}, 0\right) \rightarrow \mathbb{C}$ é uma família topologicamente trivial em $V$ se existe germe de homeomorfismo

$$
\begin{gathered}
H:\left(\mathbb{C}^{n} \times \mathbb{C}^{r}, 0\right) \rightarrow\left(\mathbb{C}^{n} \times \mathbb{C}^{r}, 0\right) \\
H(x, u)=(h(x, u), u),
\end{gathered}
$$

com $h(x, 0)=I d, h(0, u)=0$, tal que $H$ preserva $\widetilde{V}=V \times \mathbb{C}^{r}$,

$$
(F \circ H)(x, u)=F(h(x, u), u)=f(x) .
$$

Uma aplicação do Teorema 3.61 aparece no caso de famílias de funções definidas sobre uma ICIS $V$ que também satisfaz $L C(V)$ Cohen-Macaulay. Dentro destas condições apresentamos os seguintes resultados.

Proposição 3.64: Sejam $V \subset \mathbb{C}^{n}$ uma ICIS, e $F:\left(\mathbb{C}^{n} \times \mathbb{C}^{r}, 0\right) \rightarrow \mathbb{C}$ uma família de funçôes com singularidade isolada, então $E u_{f_{u}, V}(0)$ constante para a familia é equivalente à $\mu_{L}\left(f_{u}\right)$ constante para a família.

A demonstração desta proposição segue diretamente do Teorema 3.37 .

Teorema 3.65: Sejam $V \subset \mathbb{C}^{n}$ uma hipersuperfície com singularidade isolada, e $F:\left(\mathbb{C}^{n} \times \mathbb{C}^{r}, 0\right) \rightarrow$ $\mathbb{C}$ uma família de funçôes com singularidade isolada, então:

(a) $\mu_{B R}\left(f_{u}\right)$ constante para a família implica que $\mu\left(f_{u}\right), \mu_{L}\left(f_{u}\right)$ e $E u_{f_{u}, V}(0)$ são constante para a família.

(b) Se $\mu\left(f_{u}\right)$ é constante para a família, então tanto $E u_{f_{u}, V}(0)$ constante para a família como $\mu_{L}\left(f_{u}\right)$ constante para a família implicam $\mu_{B R}\left(f_{u}\right)$ constante para a família.

Demonstração: Como $V$ é uma hipersuperfície com singularidade isolada, temos $L C(V)$ CohenMacaulay. Pelo Teorema 3.61 temos

$$
\mu_{B R}\left(f_{u}\right)=m_{0} \mu\left(f_{u}\right)+m_{1}(-1)^{\operatorname{dim}_{\mathbb{C}} V} E u_{f_{u}, V_{\text {reg }}}(0)+m_{2} n_{2}
$$

aplicando o Teorema 3.37 na relação acima, obtemos,

$$
\mu_{B R}\left(f_{u}\right)=m_{0} \mu\left(f_{u}\right)+m_{1}\left[\mu_{L}\left(f_{u}\right)-\mu_{L}(l)\right]+m_{2} n_{2} .
$$

Para provar o ítem $(a)$, note que como os invariantes $\mu(f)$ e $\mu_{L}(f)$ são semi-contínuos superiormente, e como $l$ é uma forma linear genérica temos $\left[\mu_{L}(f)-\mu_{L}(l)\right] \geq 0, \operatorname{logo} \mu_{B R}\left(f_{u}\right)$ constante para a família implica a constância dos outros invariantes $\mu\left(f_{u}\right), \mu_{L}\left(f_{u}\right)$ e $E u_{f_{u}, V}(0)$.

Para provar o ítem $(b)$, note que pelas relações acima, quando $\mu\left(f_{u}\right)$ é constante para a família temos que tanto $E u_{f_{u}, V}(0)$ constante para a família como também $\mu_{L}\left(f_{u}\right)$ constante para a família implicam $\mu_{B R}\left(f_{u}\right)$ constante para a família. 
Corolário 3.66: Sejam $V \subset \mathbb{C}^{n}$ uma hipersuperfície com singularidade isolada na origem, e $F$ : $\left(\mathbb{C}^{n} \times \mathbb{C}^{r}, 0\right) \rightarrow \mathbb{C}$, uma família de funçôes com singularidade isolada, topologicamente trivial em $V$, então $\mu_{B R}\left(f_{u}\right)$ é constante para a família.

Demonstração: Como $f_{u}$ é uma família topologicamente trivial em $V$, pelos resultados de Teissier [70], temos $\mu\left(f_{u}\right)$ constante para a família, logo, pelo teorema anterior basta provar que $\mu_{L}\left(f_{u}\right)$ é constante para a família.

De fato, a fibra $f_{u}^{-1}\left(t_{0}\right)$ tem o tipo de homotopia de um buquê de esferas, e o número de Milnor de Lê é dado pelo número de esferas do buquê. Como $f_{0}=f_{u} \circ h_{u}$ temos que as fibras de $f_{u}$ e $f_{0}$ são homeomorfas, logo o número de Milnor de Lê é constante nesta família, de onde segue o resultado.

Exemplo 3.67: Sejam $V=\left\{x^{3}+y^{2}+z^{2}=0\right\}$ e $f_{u}(x, y, z)=x y+z^{2}+u x^{3}$. Os geradores de $\Theta_{V}$ são, $\xi_{1}=2 x \partial_{x}+3 y \partial_{y}+2 z \partial_{z}, \xi_{2}=-2 y \partial_{x}+3 x \partial_{y}$ e $\xi_{3}=z \partial_{y}-y \partial_{z}$, assim obtemos

$$
J_{V}\left(f_{u}\right)=\left\langle 5 x y+6 u x^{3}+4 z^{2}, 3 x^{2}-2 y^{2}-6 u x^{2} y, x z-2 y z\right\rangle
$$

Calculando a dimensão $\operatorname{dim} \mathcal{O}_{3} / J_{V}\left(f_{u}\right)$ obtemos que $\mu_{B R}\left(f_{u}\right)=6$, ou seja, constante para família, portanto os demais invariantes também são constantes na família.

Neste exemplo, $V$ e $f_{0}(x, y, z)=x y+z^{2}$ são quase-homogêneas com relação à mesma filtração $\operatorname{peso}(\mathrm{x})=2 \operatorname{peso}(\mathrm{y})=3$ e $f_{u}$ é uma deformação de $f_{0}$ por termos de grau (pesado) maiores do que o grau (pesado) de $f_{0}$. Logo, segue de [16] (ver também [56]) que a família $f_{u}$ é topologicamente trivial em $V$.

A recíproca do corolário, isto é, a questão $\mu_{B R}$ constante implica a trivialidade topológica da família é um problema aberto nesta teoria. 


\section{Obstrução de Euler de uma Aplicação}

Um passo natural para a generalização da obstrução de Euler de uma função é a obstrução de Euler de uma aplicação $f:(V, 0) \rightarrow\left(\mathbb{C}^{k}, 0\right)$, onde $(V, 0)$ é um germe de variedade analítica complexa, equidimensional de dimensão $n \geq k$. Analogamente ao caso de funções, definimos a obstrução de Euler de uma aplicação analítica $f$, em função de um $k$-referencial que depende das funções coordenadas da aplicação $f$.

A obstrução de Euler local associada a um $k$-referencial sobre uma variedade analítica foi estudada por J.-P. Brasselet, J. Seade e T. Suwa em [11]. Apresentaremos neste capítulo uma fórmula que relaciona a obstrução de Euler local associada a um $k$-referencial sobre uma variedade analítica e a obstrução de Euler de uma aplicação analítica $f:(V, 0) \rightarrow\left(\mathbb{C}^{k}, 0\right)$, onde $(V, 0)$ é um germe de variedade analítica complexa, equidimensional de dimensão $n \geq k$ [30].

\subsection{Obstrução de Euler de um $k$-campo}

Um $k$-campo é uma coleção $v^{(k)}$ de $k$ campos de vetores. Um ponto singular de $v^{(k)}$ é um ponto em que os vetores não são linearmente independentes. Um $k$-referencial é um $k$-campo sem singularidade. Dizemos que o $k$-campo $v^{(k)}$ é estratificado se cada vetor $v_{i}$ é um campo estratificado no sentido precedente.

Seja $(V, 0) \subset(M, 0)$ um germe de variedade analítica complexa, equidimensional de dimensão complexa $n$ e $(M, 0)$ um germe de variedade complexa regular de dimensão complexa $m$. Denotemos por $\left\{V_{\alpha}\right\}$ uma estratificação de Whitney de $M$ compatível com $V$. Seja $(K)$ uma triangulação de M subordinada à estratificação $\left\{V_{\alpha}\right\}$ de $M$ e $\sigma$ uma célula de dimensão real $2(m-k+1)$ de uma decomposição celular $(D)$ construída em $M$ por dualidade a partir da triangulação $(K)$. A célula $\sigma$ é transversal a todos os estratos.

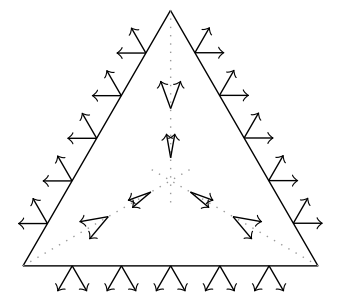


Seja $v^{(k)}$ um $k$-campo estratificado em $\sigma \cap V$ com uma singularidade isolada no baricentro $a$ de $\sigma$. O campo $v^{(k)}$ não tem singularidade sobre $\partial \sigma \cap V$. Seja $\nu: \widetilde{V} \rightarrow V$ a modificação de Nash de $V$ e $\widetilde{T}$ o fibrado de Nash. Cada componente $v_{i}$ de $v^{(k)}$ admite um levantamento $\tilde{v}_{i}$ como seção de $\widetilde{T}$ sobre $\nu^{-1}(\partial \sigma \cap V)$. O campo de $k$-referenciais $v^{(k)}$ se levanta como conjunto $\tilde{v}^{(k)}$ de $k$-seções linearmente independentes de $\widetilde{T}$ sobre $\nu^{-1}(\partial \sigma \cap V)[11]$.

Denotemos por $\operatorname{Obs}\left(\tilde{v}^{(k)}, \sigma \cap V\right)$ a classe em $H^{2(n-k+1)}\left(\nu^{-1}(\sigma \cap V),\left(\nu^{-1}(\partial \sigma \cap V)\right)\right)$ do cociclo de obstrução para estender $\tilde{v}^{(k)}$ como conjunto de $k$ seções linearmente independentes de $\widetilde{T}$ sobre $\nu^{-1}(\sigma \cap V)$.

Definição 4.1 (Definição original, ver [11]): A obstrução de Euler local $E u\left(v^{(k)}, V, \sigma\right)$ de um kcampo estratificado $v^{(k)}$ definido sobre $\sigma \cap V$ com singularidade isolada no baricentro a de $\sigma e ́$ definida como a avaliação do cociclo de obstrução $\operatorname{Obs}\left(\tilde{v}^{(k)}, \sigma \cap V\right)$ sobre a classe fundamental do par $\left[\nu^{-1}(\sigma \cap V), \nu^{-1}(\partial \sigma \cap V)\right]$. Ou seja,

$$
E u\left(v^{(k)}, V, \sigma\right)=\left\langle\operatorname{Obs}\left(\tilde{v}^{(k)}, \sigma \cap V\right),\left[\nu^{-1}(\sigma \cap V), \nu^{-1}(\partial \sigma \cap V)\right]\right\rangle .
$$

Nosso objetivo aqui é dar um método de cálculo (fórmula (4.2)) desta obstrução de Euler em função da obstrução de Euler local $E u_{V}\left(V_{\alpha}\right)$ dos estratos.

\subsection{Caso de um $k$-campo obtido por prolongamento radial.}

Em toda seqüência denotaremos $p=m-k+1$.

Em [10], é mostrado que podemos construir, sobre o $2 p$-esqueleto de $D$, denotado por $(D)^{2 p}$, pelo procedimento de extensão radial de M.-H. Schwartz, uma $k$-seção de $\cup T V_{\alpha}$, chamada $k$-campo radial e denotada por $v^{(k)}=\left(v^{(k-1)}, v_{k}\right)$ satisfazendo às seguintes propriedades em relação ao índice de M.-H. Schwartz de um $k$-campo obtido por prolongamento radial:

1. $v^{(k)}$ tem apenas pontos singulares isolados, que são os zeros do último campo de vetores $v_{k}$. Sobre $(D)^{2 p-1}, v^{(k)}$ não tem pontos singulares. Sobre $(D)^{2 p}$, o campo de $(k-1)$-referenciais $v^{(k-1)}$ não tem ponto singular.

2. Os pontos singulares de $v^{(k)}$ são os baricentros das células $\sigma^{2 p}$ de dimensão $2 p$, denotados por $a=\sigma^{2 p} \cap s$ onde $s$ é o $2(k-1)$ simplexo tal que $\sigma$ é sua célula dual, $s$ está situado no estrato de menor dimensão que encontra $\sigma$.

3. Seja $a \in V_{\alpha} \cap(D)^{2 p}$ um ponto singular de $v^{(k)}$. Se $\operatorname{dim}_{\mathbb{C}} V_{\alpha}>k-1$, o índice de $v^{(k)}$ em $a$ é igual ao índice da restrição de $v^{(k)}$ à $V_{\alpha} \cap(D)^{2 p}$, considerado como campo de $k$-referenciais tangente a $V_{\alpha}$.

Se $\operatorname{dim}_{\mathbb{C}} V_{\alpha}=k-1, \operatorname{temos} I\left(v^{(k)}, a\right)=+1$.

Mais ainda, o campo $v_{k}$ aponta para fora de certos tubos, vizinhanças de $V_{i}$ em M (Ver [10]).

Observação 4.2: Se o k-campo $v^{(k)}$ é um k-campo radial construído pelo procedimento de prolongamento radial de M.-H. Schwartz e $a \in V_{\alpha}$ uma singularidade de $v^{(k)}$, baricentro de $\sigma$, então a obstrução de Euler local Eu $\left(v^{(k)}, V, \sigma\right)$ depende apenas de $\sigma_{\alpha}=\sigma \cap V_{\alpha}$ e da restrição $v_{\alpha}^{(k)}$ de $v^{(k)} a$ $\sigma_{\alpha}$. Ela não depende da célula $\sigma$ transversal aos estratos de $V$ tal que $\sigma \cap V_{\alpha}=\sigma_{\alpha}$. 
Com efeito, se $v^{(k)}$ for obtido pelo método de prolongamento radial de M.-H. Schwartz a partir de $v_{\alpha}^{(k)}$, temos o teorema de proporcionalidade ([10]):

$$
E u\left(v^{(k)}, V, \sigma\right)=E u_{V}\left(V_{\alpha}\right) \cdot I\left(v_{\alpha}^{(k)}, a\right) .
$$

O segundo membro depende apenas de $\sigma_{\alpha}$ e $v_{\alpha}^{(k)}$ de onde obtemos a afirmação da observação acima.

\subsection{Caso de um k-campo qualquer.}

Seja $v^{(k)}$ um $k$-campo qualquer com singularidade isolada em $a$, baricentro da $2 p$-célula $\sigma$. Nos propomos aqui a dar um método de cálculo de $E u\left(v^{(k)}, V, \sigma\right)$.

Denotemos por $V_{\alpha}$ o estrato contendo $a$, e seja $v_{\alpha}^{(k)}$ a restrição de $v^{(k)}$ à $V_{\alpha}$. Denotemos por $\sigma^{\prime}$ a célula de dimensão $2(m-k+1)$, obtida a partir de $\sigma$ por homotetia de centro $a$ e raio $1 / 2$. Estendemos o campo $v_{\alpha}^{(k)}$, restrito à $\sigma^{\prime} \cap V_{\alpha}$, por prolongamento radial sobre $\sigma^{\prime}$, e obtemos um $k$-campo denotado aqui por $v_{\text {rad }}^{(k)}$ sobre $\sigma^{\prime}$.

Consideremos agora a "coroa" $C=\sigma \backslash \operatorname{Int}\left(\sigma^{\prime}\right)$. Sobre o bordo de $C, \partial \sigma^{\prime} \cup \partial \sigma$, podemos definir um $k$-campo $w^{(k)}$ por $v_{r a d}^{(k)}$ sobre $\partial \sigma^{\prime}$ e por $v^{(k)}$ sobre $\partial \sigma$.

Para cada estrato $V_{\beta}$ de dimensão complexa $n_{\beta}$ tal que $a \in \overline{V_{\beta}}$, a interseção $V_{\beta} \cap C$ tem dimensão $2\left(n_{\beta}-k+1\right)$, que é a dimensão de obstrução para a construção de um $k$-campo sem singularidades sobre $V_{\beta}$, podemos então estender $w^{(k)}$ em um $k$-campo estratificado dentro de $C$ com pontos singulares isolados $a_{i}$. Sendo assim, para podermos expressar $E u\left(v^{(k)}, V, \sigma\right)$ em função dos índices destes pontos singulares, vamos proceder por indução sobre a dimensão dos estratos $V_{\beta}$ tais que $a \in \bar{V}_{\beta}$, e utilizar o procedimento de extensão radial de M.-H. Schwartz.

Seja $V_{\beta_{1}}$ o estrato de menor dimensão tal que $a \in \overline{V_{\beta_{1}}}$ e $V_{\beta_{1}} \neq V_{\alpha}$. Podemos estender o $k$-campo $w^{(k)}$, definido sobre $\bar{V}_{\beta_{1}} \cap \partial C$, para o interior de $\bar{V}_{\beta_{1}} \cap C$ em um $k$-campo $w_{1}^{(k)}$ igual a $v_{\alpha}^{(k)}$ sobre $V_{\alpha} \cap C$, com singularidades isoladas $a_{1}^{i}$.

Em torno dos pontos $a_{1}^{i}$ estendemos $w_{1}^{(k)}$ pelo método de prolongamento radial, dentro de uma pequena bola $B_{\epsilon}\left(a_{1}^{i}\right) \cap \sigma$. O campo obtido, ainda denotado por $w_{1}^{(k)}$ tem o mesmo índice no ponto $a_{1}^{i}$ que sua restrição à $V_{\beta_{1}}$, e será denotado por $I\left(w_{1}^{(k)}, a_{1}^{i}\right)$.

De [10], temos :

$$
E u\left(w_{1}^{(k)}, V, a_{1}^{i}\right)=E u_{V}\left(V_{\beta_{1}}\right) \cdot I\left(w_{1}^{(k)}, a_{1}^{i}\right) .
$$

Explicitaremos agora a passagem de $V_{\beta_{1}}$ para o estrato seguinte $V_{\beta_{2}}$, e vamos proceder a demonstração por recorrência sobre as dimensões dos estratos $V_{\beta}$ que $a \in \bar{V}_{\beta}$. Nesta demonstração as etapas seguintes são idênticas à passagem de $V_{\beta_{1}}$ para $V_{\beta_{2}}$.

Consideremos então o estrato $V_{\beta_{2}}$ de dimensão imediatamente superior a de $V_{\beta_{1}}$ e tal que $a \in \bar{V}_{\beta_{2}}$. Consideremos o subespaço

$$
X_{2}=\left(\bar{V}_{\beta_{2}} \cap C\right) \backslash \cup_{i} \operatorname{Int}\left(B_{\epsilon}\left(a_{1}^{i}\right)\right)
$$

dentro de $\bar{V}_{\beta_{2}}$. 


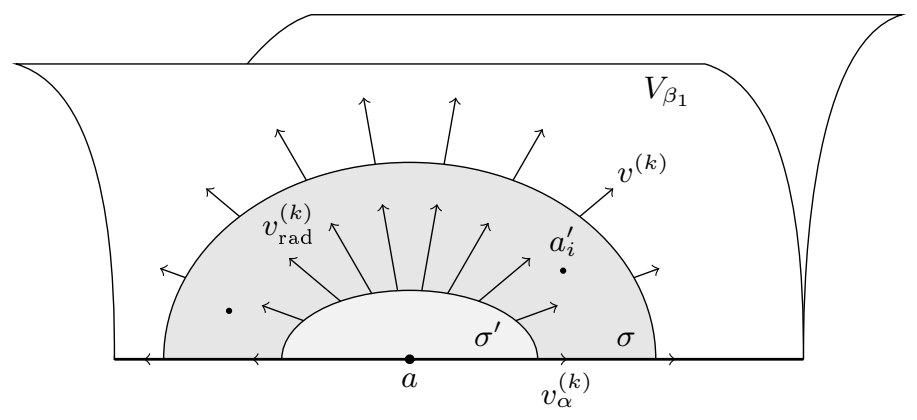

O bordo de $X_{2}$ é constítuido de: $V_{\beta_{2}} \cap \partial C$ sobre o qual o $k$-campo $w^{(k)}$ está definido, de $\left(\bar{V}_{\beta_{1}} \cap C\right) \backslash \cup_{i} \operatorname{Int}\left(B_{\epsilon}\left(a_{1}^{i}\right)\right)$ e de $\cup_{i}\left(\bar{V}_{\beta_{2}} \cap \partial B_{\epsilon}\left(a_{1}^{i}\right)\right)$ sobre o qual o $k$-campo $w_{1}^{(k)}$ está definido. Podemos então estender o campo assim definido sobre o bordo de $X_{2}$ ao interior de $X_{2}$, a um campo $w_{2}^{(k)}$ igual a $v_{\alpha}^{(k)}$ sobre $V_{\alpha} \cap C$, com singularidades isoladas $a_{2}^{i}$.

A demonstração por recorrência continua assim da mesma forma para todos os estratos $V_{\beta}$ tais que $a \in \bar{V}_{\beta}$.

Obtemos finalmente sobre $V \cap C$ um $k$-campo, denotado por $w^{(k)}$, igual a $v_{\alpha}^{(k)}$ sobre $V_{\alpha} \cap C$, com singularidades isoladas $a_{j}^{i}$ de índice $I\left(w^{(k)}, a_{j}^{i}\right)$ e situados dentro dos estratos $V_{\beta_{j}}$ tais que $V_{\alpha} \subset \bar{V}_{\beta_{j}}$. Temos:

$$
E u\left(w^{(k)}, V, \sigma_{j}^{i}\right)=E u_{V}\left(V_{\beta_{j}}\right) I\left(w^{(k)}, a_{j}^{i}\right),
$$

onde $\sigma_{j}^{i}=B_{\epsilon}\left(a_{j}^{i}\right) \cap \sigma$. O campo definido por $w^{(k)}$ dentro de $C$ coincide com $v^{(k)}$ sobre $\partial \sigma$. Denotemos ainda por $w^{(k)}$ o campo definido dentro de $\sigma$ como sendo $w^{(k)}$ dentro de $C$ e $v_{\text {rad }}^{(k)}$ dentro de $\sigma^{\prime}$. Temos então :

$$
E u\left(v^{(k)}, V, \sigma\right)=E u\left(v_{r a d}^{(k)}, V, \sigma^{\prime}\right)+\sum_{\beta_{j}, i} E u\left(w^{(k)}, V, a_{j}^{i}\right) .
$$

Nesta igualdade, todos os membros da direita se calculam pela fórmula (4.1). Temos então:

$$
E u\left(v^{(k)}, V, \sigma\right)=E u_{V}\left(V_{\alpha}\right) I\left(v_{\alpha}^{(k)}, a\right)+\sum_{\beta_{j}} E u_{V}\left(V_{\beta_{j}}\right)\left(\sum_{i} I\left(w^{(k)}, a_{j}^{i}\right)\right) .
$$

\subsection{Obstrução de Euler de uma Aplicação Analítica}

Seja como anteriormente $(V, 0) \subset(M, 0)$ um germe de variedade analítica complexa, equidimensional de dimensão complexa $n$ e $(M, 0)$ um germe de variedade complexa regular de dimensão complexa $m$. Denotemos por $\left\{V_{\alpha}\right\}$ uma estratificação de Whitney de $M$ compatível com $V$. Seja $(K)$ uma triangulação de $V$ subordinada à estratificação $\left\{V_{\alpha}\right\}$ e $(D)$ uma decomposição celular dual de $(K)$. Suponhamos que $\{0\}$ seja o baricentro de um $(k-1)$-simplexo $s$ de $(K)$. Denotaremos $\sigma$ a célula de $(D)$ de dimensão real $2(m-k+1)$ dual de $s$. O ponto $\{0\}$ é então também baricentro de $\sigma$.

Denotemos por $U$ uma vizinhança de 0 em $M$ e, para um inteiro positivo $k \leq n, f: V \rightarrow \mathbb{C}^{k}$,

$$
f(z)=\left(f_{1}(z), f_{2}(z), \ldots, f_{k}(z)\right)
$$

aplicação analítica, restrição de $F: U \rightarrow \mathbb{C}^{k}$, onde

$$
F(z)=\left(F_{1}(z), F_{2}(z), \ldots, F_{k}(z)\right)
$$


Seguindo a construção de [9], detalhada no capítulo 3 , podemos associar à cada $f_{i}$ um campo estratificado denotado por $\bar{\nabla}_{V} f_{i}(z)$.

Seja $\Sigma f$ o conjunto singular de $f$. O núcleo $\operatorname{ker}\left(d F_{z}\right)$ é transversal ao espaço tangente $T_{z} V_{\alpha}(z)$ em todos os pontos $z \in V \backslash\{\Sigma f\}$, onde $V_{\alpha}(z)$ designa o estrato $V_{\alpha}$ de $V$ que contém o ponto $z$. Então temos que $k e r\left(d F_{z}\right)+T_{z} V_{\alpha}(z)=T_{z} U$ que se identifica com $\mathbb{C}^{m}$, e como $\operatorname{dim}\left(k e r\left(d F_{z}\right)\right)=m-k$ obtemos $\operatorname{dim}\left(T_{z} V_{\alpha}(z)\right) \geq k$, ou seja, $V \backslash\{\Sigma f\}$ está contido na união dos estratos de $V$ tais que $\operatorname{dim} V_{\alpha} \geq k$

Seja $p_{\alpha}: \mathbb{C}^{m} \simeq T_{z} U \rightarrow T_{z} V_{\alpha}(z)$ a projeção canônica sobre $T_{z} V_{\alpha}(z)$, onde $z \in V \backslash\{\Sigma f\}$. Podemos definir $\bar{\nabla}_{V} f_{1}(z)$ como $p_{\alpha}\left(\bar{\nabla}_{V} f_{1}(z)\right)$, que é um vetor não nulo (ver seção 3.2). Para $i$ tal que $1<i \leq k$ fazemos por recorrência a seguinte construção: Associamos à $f_{i}(z)$ o campo $\bar{\nabla}_{V} f_{i}(z)=p_{\alpha}\left(\bar{\nabla} f_{i}(z)\right)$, se o conjunto $\left.\left\{\bar{\nabla}_{V} f_{1}(z), \bar{\nabla}_{V} f_{2}(z), \ldots, \bar{\nabla}_{V} f_{i}(z)\right)\right\}$ for linearmente independente. Caso contrário, definimos $f_{i}^{\prime}(z)=f_{i}+\epsilon_{i} l_{i}$, onde $l_{i}:(V, 0) \rightarrow(\mathbb{C}, 0)$ é uma transformação linear genérica, com $\epsilon_{i}$ suficientemente pequeno de tal forma com que o conjunto

$$
\left\{\bar{\nabla}_{V} f_{1}(z), \bar{\nabla}_{V} f_{2}(z), \ldots, \bar{\nabla}_{V} f_{i-1}(z), p_{\alpha}\left(\bar{\nabla} f_{i}^{\prime}(z)\right)\right\}
$$

seja linearmente independente. Definimos então $\bar{\nabla}_{V} f_{i}(z)=p_{\alpha}\left(\bar{\nabla} f_{i}^{\prime}(z)\right)$. Note que, para $\left\|\left(0, \epsilon_{2}, \ldots, \epsilon_{k}\right)\right\|$ suficientemente pequeno, $f$ é homotópico à aplicação :

$$
g(z)=\left(f_{1}(z), f_{2}(z)+\epsilon_{2} l_{2}, \ldots, f_{k}(z)+\epsilon_{k} l_{k}\right) .
$$

Denotemos por $\bar{\nabla}_{V}^{(k)} f$ o $k$-campo $\left(\bar{\nabla}_{V} f_{1}(z), \bar{\nabla}_{V} f_{2}(z), \ldots, \bar{\nabla}_{V} f_{k}(z)\right)$, que é um $k$-campo estratificado sem singularidades sobre $\left(\partial B_{\epsilon} \cap V \backslash\{\Sigma f\}\right)$, onde $B_{\epsilon}$ é uma bola em $M$ centrada na origem.

Quando $V$ for uma variedade regular e $\operatorname{dim} \Sigma f \leq k-1$, podemos sempre escolher a célula $\sigma$ tal que:

$$
\Sigma f \cap \partial \sigma=\emptyset .
$$

Quando $V$ for uma variedade singular, $\Sigma$ a parte singular de $V$ e $V_{\text {reg }}$ a parte regular, se $\operatorname{dim}_{\mathbb{C}} \Sigma \leq$ $k-1$, e se $\left.f\right|_{V_{\text {reg }}}$ for tal que $\left.\operatorname{dim}_{\mathbb{C}} \Sigma f\right|_{V_{\text {reg }}} \leq k-1$, existe $\sigma$ tal que a relação $(\delta)$ seja satisfeita.

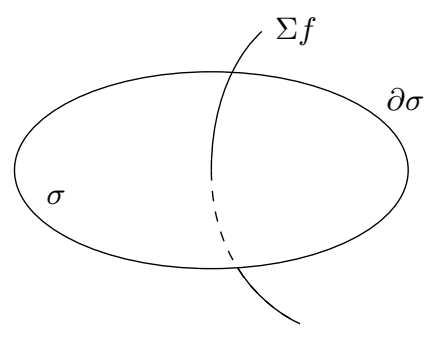

Definição 4.3: Seja $(V, 0) \subset(M, 0)$ um germe de variedade analítica complexa, equidimensional, de dimensão complexa $n$, contida em $(M, 0)$ germe de variedade analítica complexa regular de dimensão complexa $m$. Seja $f:(V, 0) \rightarrow\left(\mathbb{C}^{k}, 0\right)$, analítica, $f(z)=\left(f_{1}(z), f_{2}(z), \ldots, f_{k}(z)\right)$ e $\Sigma f$ o conjunto singular de $f$. Dizemos que $f$ satisfaz à condição $(\delta)$ se existe uma célula $\sigma$ de baricentro 0 , de dimensão real $2(m-k+1)$ de uma decomposição celular $(D)$ de $M$, tal que:

$$
\Sigma f \cap \partial \sigma=\emptyset .
$$


A célula $\sigma$ de dimensão real $2(m-k+1)$ tendo o ponto 0 como baricentro, é transversal na origem, isso quer dizer que é transversal a todos os estratos $V_{\alpha} \neq\{0\}$ de $V$ tais que $0 \in \bar{V}_{\alpha}$, isto significa que $\operatorname{codim}\left(\sigma \cap V_{\alpha}\right)=\operatorname{codim} \sigma+\operatorname{codim} V_{\alpha}$ para todos os estratos $V_{\alpha}$. Denotamos por $V^{\sigma}$ o conjunto $\sigma \cap V$. Se $f$ satisfaz à condição $(\delta)$ para a célula $\sigma$, o campo $\bar{\nabla}_{V}^{(k)} f$ se levanta como um conjunto de $k$ seções linearmente independentes $\widetilde{\bar{\nabla}}_{V}^{(k)} f$ de $\widetilde{T}$ sobre $\nu^{-1}\left(\partial V^{\sigma}\right)$. Seja $\xi \in H^{2(n-k+1)}\left(\nu^{-1}\left(V^{\sigma}\right), \nu^{-1}\left(\partial V^{\sigma}\right)\right)$ o cociclo de obstrução para a extensão de $\widetilde{\nabla}_{V}^{(k)} f$ como conjunto de $k$ seções linearmente independentes de $\widetilde{T}$ sobre $\nu^{-1}\left(V^{\sigma}\right)$.

Definição 4.4: Dentro das condiçôes anteriores, definimos a obstrução de Euler de $f$, denotada por $E u_{f, V}(\sigma)$, como a avaliação do cociclo $\xi$ sobre a classe fundamental do par $\left[\nu^{-1}\left(V^{\sigma}\right), \nu^{-1}\left(\partial V^{\sigma}\right)\right]$. Ou seja,

$$
E u_{f, V}(\sigma)=\left\langle\xi,\left[\nu^{-1}\left(V^{\sigma}\right), \nu^{-1}\left(\partial V^{\sigma}\right)\right]\right\rangle
$$

No caso em que tomamos $k=1$, recuperamos a obstrução de Euler de $f$ definida em [9] e também detalhada neste trabalho.

\subsection{Teorema Principal}

Lembremos que $f=\left(f_{1}, \ldots, f_{k}\right): V \rightarrow \mathbb{C}^{k}$ é a restrição de uma aplicação analítica $F=\left(F_{1}, \ldots, F_{k}\right)$ : $U \rightarrow \mathbb{C}^{k}$ onde $U$ é uma vizinhança de 0 em $M$. Denotemos ainda $B_{\epsilon}$ uma bola centrada em 0 e de raio $\epsilon$. Podemos supor que

$$
\sigma_{f}=B_{\epsilon} \cap F_{1}^{-1}(0) \cap F_{2}^{-1}(0) \cap \cdots \cap F_{k-1}^{-1}(0)
$$

é transversal à $V$. De fato, se $\sigma_{f}$ não for transversal à $V$ (quer dizer, não é transversal a todos os estratos $V_{\alpha}$ de $V$ diferentes da origem), podemos tomar $F_{i}^{\prime}=F_{i}+\lambda_{i} l_{i}$, onde $l_{i}:(V, 0) \rightarrow(\mathbb{C}, 0)$ é uma transformação linear genérica, tal que $\cap F_{i}^{\prime-1}(0)$ seja transversal à $V$.

O conjunto $\sigma_{f}$ pode ser identificado localmente com uma célula de dimensão $2(m-k+1)$ de uma decomposição celular dual $(D)$, construída a partir de uma triangulação $(K)$ de $M$ subordinada à estratificação $\left\{V_{\alpha}\right\}$, onde 0 é o baricentro da célula $\sigma$.

O próximo resultado o resultado principal deste capítulo, generalização do resultado de [9].

Teorema 4.5: Seja $(V, 0) \subset(M, 0)$ um germe de variedade analitica complexa, equimensional de dimensão complexa $n$ contido em $(M, 0)$ germe de variedade regular complexa de dimensão complexa $m$, e seja $\left\{V_{\alpha}\right\}$ uma estratificação de Whitney de $M$ compativel com $V$. Para um inteiro positivo $k \leq n$, seja $f: V \rightarrow \mathbb{C}^{k}$, analítica, satisfazendo a condição $(\delta)$ para a célula $\sigma=\sigma_{f}$ de dimensão real $2(m-k+1)$ de uma decomposição celular $(D)$ e tal que 0 seja baricentro de $\sigma$. Seja $v^{(k)}=\left(v^{(k-1)}, v_{k}\right)$ um campo de $k$-referenciais sem singularidades sobre $\partial V^{\sigma}$ tal que $v_{k}$ seja um campo radial no sentido usual, então temos que:

$$
E u\left(v^{(k)}, V, \sigma\right)=\left(\sum_{\alpha} E u_{V}\left(V_{\alpha}\right) \cdot \chi\left(V_{\alpha} \cap B_{\epsilon} \cap f^{-1}\left(z_{0}\right)\right)\right)+E u_{f, V}(\sigma)
$$

onde $\left\{V_{\alpha}\right\}$ descreve o conjunto de estratos de $V$ tais que $0 \in \bar{V}_{\alpha}$. 
Demonstração: Seja $\sigma=\sigma_{f}$ a $2(m-k+1)$ célula construída acima, e seja $\sigma^{\prime} \subset \sigma$ obtida por homotetia de $\sigma$, por uma homotetia de centro em 0. Denotemos $V^{\sigma}=\sigma \cap V$ e $V^{\sigma^{\prime}}=\sigma^{\prime} \cap V$.

Seja $f:(V, 0) \rightarrow\left(\mathbb{C}^{k}, 0\right)$, analítica,

$$
f(z)=\left(f_{1}(z), f_{2}(z), \ldots, f_{k}(z)\right) .
$$

Mostraremos primeiramente que podemos construir um campo de $k$-referenciais "adaptado" à $f$, $v_{f}^{(k)}=\left(v_{f}^{(k-1)}, v\right)$ sobre $V^{\sigma} \backslash \operatorname{Int}\left(\sigma^{\prime}\right)$ tal que:

(i) $v_{f}^{(k)}$ coincide com $\bar{\nabla}_{V}^{(k)} f$ sobre $\partial V^{\sigma^{\prime}}$ e com $v^{(k)}$ sobre $\partial V^{\sigma}$;

(ii) $v$ é tangente à $f^{-1}\left(z_{0}\right) \cap V^{\sigma}$, onde $z_{0} \in \mathbb{C}^{k} \backslash\{f(\Sigma f)\}$ é um valor regular de $f$ próximo da origem;

(iii) $v_{f}^{(k)}$ tem apenas um número finito de singularidades sobre $V^{\sigma} \backslash\{0\}$, todas situadas em $f^{-1}\left(z_{0}\right)$;

(iv) em cada singularidade $a$ de $v_{f}^{(k)}$ o campo $v$ é transversalmente radial ao estrato contendo $a$.

Consideremos a "coroa" $C=\sigma \backslash \operatorname{Int}\left(\sigma^{\prime}\right)$. Sobre o bordo de $C, \partial \sigma^{\prime} \cup \partial \sigma$ podemos definir o $k$-campo $v_{C}^{(k)}$ como sendo $\bar{\nabla}_{V}^{(k)} f$ sobre $\partial \sigma^{\prime}$ e $v^{(k)}$ sobre $\partial \sigma$. Como no caso do $k$-campo $v^{(k)}$ da construção 4.3 , podemos prolongar $v_{C}^{(k)}$ sobre $C$ em um $k$-campo pelo procedimento de prolongamento radial de M.-H. Schwartz, denotemos ainda por $v_{C}^{(k)}$ a extensão obtida. O $k$-campo $v_{C}^{(k)}$ sobre $C \cap V$ coincide com $\bar{\nabla}_{V}^{(k)} f$ sobre $\partial \sigma^{\prime} \cap V$.

Chamemos de $w_{f}^{(k)}$ o $k$-campo obtido sobre $\sigma \cap V$, igual à $v_{C}^{(k)}$ sobre $C \cap V$ e à $\bar{\nabla}_{V}^{(k)} f$ sobre $\sigma^{\prime} \cap V$.

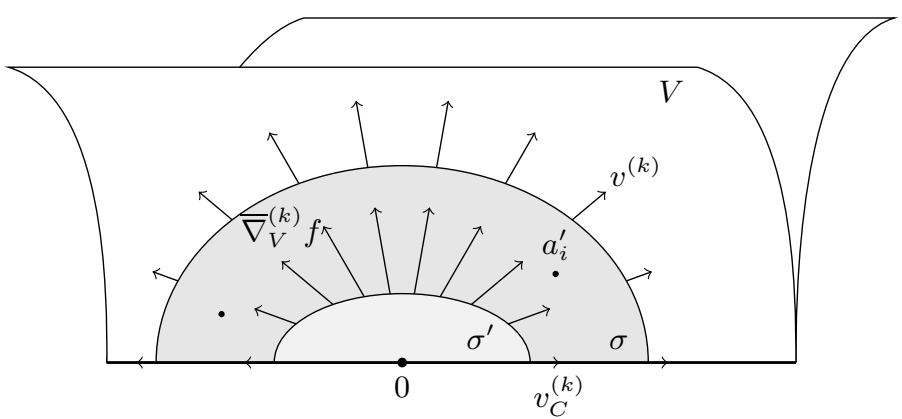

Consideremos a última função coordenada $f_{k}: V^{\sigma} \rightarrow \mathbb{C}$, sabemos por [9] que, se $\sigma$ e $\sigma^{\prime}$ são suficientemente pequenas, existe um campo de vetores estratificado $v$ sobre $V^{\sigma} \backslash \operatorname{Int}\left(\sigma^{\prime}\right)$ tal que:

a) $v$ coincide com $\bar{\nabla}_{V} f_{k}(z)$ sobre $\partial V^{\sigma^{\prime}}$ e é um campo radial sobre $\partial V^{\sigma}$;

b) $v$ é tangente à $f_{k}^{-1}\left(t_{0}\right)$ para $t_{0}$ proximo de 0 ;

c) $v$ tem apenas um número finito de singularidades, todas situadas dentro de $f_{k}^{-1}\left(t_{0}\right)$;

d) em cada singularidade $a$, o campo $v$ é radial relativamente ao estrato contendo $a$. 
Trocando a última coordenada de $w_{f}^{(k)}$ pelo campo $v$ construído acima, obtemos um campo de $k$-referenciais satisfazendo às condições desejadas. Denotemos por $v_{f}^{(k)}$ o $k$-campo obtido.

O $k$-campo $v^{(k)}$, respectivamente o $k$-campo $v_{f}^{(k)}$, admite um levantamento $\widetilde{v}^{(k)}$, respectivamente $\widetilde{v}_{f}^{(k)}$, como conjunto de $k$ seções linearmente independentes de $\widetilde{T}$ sobre $\nu^{-1}\left(\partial V^{\sigma}\right)$.

Denotemos por $\operatorname{Obs}\left(\tilde{v}^{(k)}, \nu^{-1}\left(\partial V^{\sigma}\right)\right)$, respectivamente por $\operatorname{Obs}\left(\tilde{v}_{f}^{(k)}, \nu^{-1}\left(\partial V^{\sigma}\right)\right)$, o cociclo de obstrução para estender $\tilde{v}^{(k)}$, respectivamente $\tilde{v}_{f}^{(k)}$, sobre $\nu^{-1}\left(V^{\sigma}\right)$ a partir de $\nu^{-1}\left(\partial V^{\sigma}\right)$.

Lembrando que os $k$-campos $v^{(k)}$ e $v_{f}^{(k)}$ são homotópicos sobre $\partial V^{\sigma}$, temos que:

$$
\operatorname{Obs}\left(\tilde{v}^{(k)}, \nu^{-1}\left(\partial V^{\sigma}\right)\right)=\operatorname{Obs}\left(\tilde{v}_{f}^{(k)}, \nu^{-1}\left(\partial V^{\sigma}\right)\right)
$$

Temos então que:

$$
\begin{aligned}
\operatorname{Obs}\left(\tilde{v}^{(k)}, \nu^{-1}\left(\partial V^{\sigma}\right)\right) & =\operatorname{Obs}\left(\tilde{v}_{f}^{(k)}, \nu^{-1}\left(\partial V^{\sigma^{\prime}}\right)\right) \\
& +\operatorname{Obs}\left(\tilde{v}_{f}^{(k)}, \nu^{-1}\left(V \cap \partial\left(\sigma \backslash \operatorname{Int}\left(\sigma^{\prime}\right)\right)\right)\right) .
\end{aligned}
$$

Como o $k$-campo $v_{f}^{(k)}$ é adaptado à $f$, pela condição $(i)$ da construção de $v_{f}^{(k)}$ e por definição (Definição 4.4) temos (por abuso de linguagem entre a classe e a avaliação):

$$
E u\left(v^{(k)}, V, \sigma\right)=E u_{f, V}(\sigma)+\operatorname{Obs}\left(\tilde{v}_{f}^{(k)}, \nu^{-1}\left(V \cap \partial\left(\sigma \backslash \operatorname{Int}\left(\sigma^{\prime}\right)\right)\right)\right) .
$$

Pela condição $(i i i)$ da construção de $v_{f}^{(k)}$ acima, a contribuição de cociclo de obstrução

$$
\operatorname{Obs}\left(\tilde{v}_{f}^{(k)}, \nu^{-1}\left(V \cap \partial\left(\sigma \backslash \operatorname{Int}\left(\sigma^{\prime}\right)\right)\right)\right)
$$

está concentrada dentro de $\nu^{-1}\left(f^{-1}\left(z_{0}\right) \cap V^{\sigma}\right)$. Pela condição $(i v)$ e pelo Teorema de Proporcionalidade (ver fórmula 4.1), a contribuição de cada singularidade $a_{l}$ de $v_{f}^{(k)}$ é $\operatorname{E} u_{V}\left(a_{l}\right) \operatorname{Ind}\left(v_{f}^{(k)}, a_{l}\right)$, onde $E u_{V}\left(a_{l}\right)$ é constante e igual à $E u_{V}\left(V_{\alpha}\right)$ para toda singularidade $a_{l}$ situada dentro de $V_{\alpha}$ (Observação 2.13). A avaliação do último termo de (5) é igual à :

$$
\sum_{a_{l} \in V^{\sigma}} E u_{V}\left(a_{l}\right) \operatorname{Ind}\left(v_{f}^{(k)}, V, a_{l}\right)=\sum_{\alpha} E u_{V}\left(V_{\alpha}\right)\left(\sum_{a_{l} \in V_{\alpha}^{\sigma}} \operatorname{Ind}\left(v_{f}^{(k)}, V_{\alpha}, a_{l}\right)\right) .
$$

Mas, as singularidades de $v_{f}^{(k)}$ são na verdade as singularidades de $v$, que estão todas situadas dentro de $f^{-1}\left(z_{0}\right)$, pela condição $(i i)$ da construção de $v_{f}^{(k)}$, temos então:

$$
\sum_{a_{l} \in V_{\alpha} \cap \sigma} \operatorname{Ind}\left(v_{f}^{(k)}, V_{\alpha}, a_{l}\right)=\sum_{a_{l} \in V_{\alpha} \cap \sigma} \operatorname{Ind}\left(v, V_{\alpha} \cap \sigma, a_{l}\right)=\chi\left(V_{\alpha} \cap \sigma \cap f^{-1}\left(z_{0}\right)\right) .
$$

Finalmente, observando que $E u_{f, V}\left(\sigma^{\prime}\right)=E u_{f, V}(\sigma)$, e como

$$
\sigma=B_{\epsilon} \cap F_{1}^{-1}(0) \cap F_{2}^{-1}(0) \cap \cdots \cap F_{k-1}^{-1}(0),
$$

segue o teorema. 


\subsection{Caso de Variedades Regulares}

No caso em que $V$ é uma variedade regular, obtemos as seguintes fórmulas:

Corolário 4.6: Seja $f:\left(\mathbb{C}^{n}, 0\right) \rightarrow\left(\mathbb{C}^{k}, 0\right), n \geq k$, um germe analítico finitamente determinado ([21, 67]). Temos então que $f^{-1}(0)$ é uma ICIS. Em particular, a dimensão (complexa) $\operatorname{dim}_{\mathbb{C}}\left(f^{-1}(0)\right)=$ $n-k$.

Utilizando o teorema principal (Teorema 4.5) temos

$$
E u\left(v^{(k)}, \mathbb{C}^{n}, \sigma\right)=\chi\left(f^{-1}\left(z_{0}\right) \cap B_{\epsilon}(0)\right)+E u_{f, \mathbb{C}^{n}}(\sigma),
$$

e, como $E u\left(v^{(k)}, \mathbb{C}^{n}, \sigma\right)=1$, logo:

$$
E u_{f, \mathbb{C}^{n}}(\sigma)=1-\chi\left(f^{-1}\left(z_{0}\right) \cap B_{\epsilon}(0)\right) .
$$

Como $f^{-1}(0)$ é uma ICIS, temos

$$
\chi\left(f^{-1}\left(z_{0}\right) \cap B_{\epsilon}(0)\right)=1+(-1)^{n-k} \mu\left(f^{-1}(0)\right)
$$

onde $\mu\left(f^{-1}(0)\right)$ é o número de Milnor de $f^{-1}(0)$. Obtemos então

$$
E u_{f, \mathbb{C}^{n}}(\sigma)=(-1)^{n-k+1} \mu\left(f^{-1}(0)\right) .
$$

Corolário 4.7: Seja $f:\left(\mathbb{C}^{n}, 0\right) \rightarrow\left(\mathbb{C}^{k}, 0\right)$ analítica, $n \geq k$, tal que :

$$
f\left(x_{1}, x_{2}, \ldots, x_{n}\right)=\left(x_{1}, x_{2}, \ldots, x_{k-1}, g(x)\right),
$$

onde $x=\left(x_{1}, \ldots, x_{n}\right)$. Suponhamos que

$$
g_{0}\left(x_{k}, \ldots, x_{n}\right)=g\left(0, \ldots, 0, x_{k}, \ldots, x_{n}\right)
$$

seja uma função com singularidade isolada na origem. Temos:

$$
E u_{f, \mathbb{C}^{n}}(\sigma)=(-1)^{n-k+1} \mu\left(g_{0}\right),
$$

onde $\mu\left(g_{0}\right)$ é o número de Milnor de $g_{0}$ na origem.

Demonstração: O conjunto singular de $f$ é dado por:

$$
\Sigma f=\left\{\left(x_{1}, x_{2}, \ldots, x_{n-1}, x_{n}\right): \partial_{k} g(x)=\partial_{k+1} g(x)=\cdots=\partial_{n} g(x)=0\right\},
$$

Podemos tomar

$$
\sigma=B_{\epsilon} \cap(0,0, \ldots, 0) \times \mathbb{C}^{n-k+1},
$$

temos $f^{-1}\left(0,0, \ldots, 0, t_{0}\right) \cap \sigma=g_{0}^{-1}\left(t_{0}\right) \cap \sigma$. Como

$$
g_{0}\left(x_{k}, \ldots, x_{n}\right)=g\left(0, \ldots, 0, x_{k}, \ldots, x_{n}\right)
$$

é uma função com singularidade isolada na origem, a condição $(\delta)$ está satisfeita e portanto, pela fórmula do teorema 4.5 segue que:

$$
E u_{\mathbb{C}^{n}}(0)=\chi\left(\sigma \cap f^{-1}\left(0, \ldots, t_{0}\right)\right)+E u_{f, \mathbb{C}^{n}}(\sigma),
$$


mas

$$
\chi\left(\sigma \cap f^{-1}\left(0, \ldots, 0, t_{0}\right)\right)=\chi\left(\sigma \cap g_{0}^{-1}\left(t_{0}\right)\right),
$$

então,

$$
E u_{f, \mathbb{C}^{n}}(\sigma)=(-1)^{n-k+1} \mu\left(g_{0}\right)
$$

Observação 4.8: Notemos que um germe $f(x)$ de coposto 1, do tipo acima, ou seja,

$$
f(x)=\left(x_{1}, \ldots, x_{k-1}, g(x)\right),
$$

onde $x=\left(x_{1}, \ldots, x_{n}\right)$, é $\mathcal{K}$-equivalente (cf Seção 1.3) ao germe

$$
h(x)=\left(x_{1}, \ldots, x_{k-1}, g_{0}\left(x_{k}, \ldots, x_{n}\right)\right),
$$

porque os ideais gerados pelas funções coordenadas dos dois germes são iguais. Sendo assim, o Corolário 4.7 se aplica por exemplo, para todas as singularidades do tipo $A_{l}$.

Exemplo 4.9: Seja $f:\left(\mathbb{C}^{3}, 0\right) \rightarrow\left(\mathbb{C}^{2}, 0\right)$ onde $f(x, y, z)=(x, g(y, z))$, onde $g(y, z)=y^{2}+z^{r+1}$ o conjunto singular de $f$ é $\Sigma f=\mathbb{C} \times\{0,0\}$, pela fórmula do Corolário 4.7 temos:

$$
E u_{f, \mathbb{C}^{3}}(\sigma)=(-1)^{2} \mu(g)=\mu(g)=r
$$

\subsection{Caso Equidimensional}

Sejam $(V, 0)$ germe de variedade analítica, reduzida, equidimensional de dimensão complexa $d$, dotado de uma estratificação de Whitney (tal que $V_{\text {reg }}$ seja um dos estratos) e $f:(V, 0) \rightarrow \mathbb{C}^{d}$ germe de aplicação analítica finita. Para todo $z_{0} \in \mathbb{C}^{d}$ suficientemente próximo da origem e tal que $f^{-1}\left(z_{0}\right)$ é transversal à $V$, temos pelo Teorema 4.5 ,

$$
E u\left(v^{(d)}, V, \sigma\right)=\left(\sum_{\alpha} E u_{V}\left(V_{\alpha}\right) \cdot \chi\left(V_{\alpha} \cap B_{\epsilon} \cap f^{-1}\left(z_{0}\right)\right)\right)+E u_{f, V}(\sigma) .
$$

Podemos supor que $f^{-1}\left(z_{0}\right)$ não encontra os estratos de dimensão menor do que $d$, ou seja, encontra apenas o estrato $V_{\text {reg }}$ e como $E u_{V}\left(V_{\text {reg }}\right)=1$, podemos simplificar a fórmula acima da seguinte forma,

$$
E u\left(v^{(d)}, V, \sigma\right)=\chi\left(V \cap B_{\epsilon} \cap f^{-1}\left(z_{0}\right)\right)+E u_{f, V}(\sigma) .
$$

Mas por definição $\operatorname{grau}(f)=\chi\left(V \cap B_{\epsilon} \cap f^{-1}\left(z_{0}\right)\right)$, de onde obtemos,

$$
E u\left(v^{(d)}, V, \sigma\right)=\operatorname{grau}(f)+E u_{f, V}(\sigma) .
$$

Um caso particular interessante é quando $\operatorname{dim}(V)=1$, ou seja, curvas espaciais, não necessariamente ICIS. Em [55], J. J. Nuño-Balesteros e J. N. Tomazella mostraram que se $(V, 0)$ é um germe de curva reduzida e $f:(V, 0) \rightarrow(\mathbb{C}, 0)$ um germe de função finita sobre $V$, então

$$
\mu_{G}(f)=\mu(V, 0)+\operatorname{grau}(f)-1,
$$

onde $\mu_{G}(f)$ denota o número de Milnor devido a V. Goryunov, D. Mond e D. van Straten (Observação 3.38) e $\mu(V, 0)$ denota o número de Milnor da curva ([55], Teorema 2.6). 
Proposição 4.10: Sejam $(V, 0) \subset \mathbb{C}^{n}$ germe de curva analítica reduzida e $f:(V, 0) \rightarrow \mathbb{C}$, então

$$
\mu_{G}(f)=m_{1}(V, 0)-E u_{f, V}(0),
$$

onde $m_{1}(V, 0)$ denota a 1-ésima variedade polar de $V$ na origem.

Demonstração: Das relações obtidas acima sabemos que

$$
E u\left(v^{(d)}, V, \sigma\right)=\operatorname{grau}(f)+E u_{f, V}(\sigma) .
$$

Como $\operatorname{dim}_{\mathbb{C}}(V, 0)=1$, a obstrução de Euler $E u\left(v^{(d)}, V, \sigma\right)$ se reduz a $E u_{V}(0)$ e temos então:

$$
E u_{V}(0)=\operatorname{grau}(f)+E u_{f, V}(0) .
$$

Segue da fórmula de Lê e Teissier (Teorema 2.11) que $E u_{V}(0)=m_{0}(V, 0)$, assim isolando grau $(f)$ obtemos a relação $\operatorname{grau}(f)=m_{0}(V, 0)-E u_{f, V}(0)$.

Pelo Teorema 2.6 de [55] temos a relação $\mu_{G}(f)=\mu(V, 0)+\operatorname{grau}(f)-1$, ou seja, $\operatorname{grau}(f)=$ $\mu_{G}(f)-\mu(V, 0)+1$.

Sendo assim, $m_{0}(V, 0)-E u_{f, V}(0)=\mu_{G}(f)-\mu(V, 0)+1$, e isolando $\mu_{G}(f)$ temos $\mu_{G}(f)=$ $\left(m_{0}(V, 0)+\mu(V, 0)-1\right)-E u_{f, V}(0)$. Mas pelo Corolário 3.2 de [55] temos que $m_{1}(V, 0)=m_{0}(V, 0)+$ $\mu(V, 0)-1$, de onde segue o resultado.

Observação 4.11: Quando o germe de curva $(V, 0)$ for uma ICIS, podemos escrever a fórmula acima da seguinte forma, Euf,V $(0)=(-1)\left[\mu_{G}(f)-m_{1}(V, 0)\right]$, mas $m_{1}(V, 0)=\mu_{G}(l)$, onde l é uma projeção genérica (ver [55], Definição 3.1), ou seja,

$$
E u_{f, V}(0)=(-1)\left[\mu_{G}(f)-\mu_{G}(l)\right]
$$

de onde recuperamos a fórmula de J. Seade, M. Tibar e A. Verjovsky (Teorema 3.37).

No caso em que $(V, 0)$ é uma $I C I S$ de dimensão complexa $d$, motivados pela fórmula de NuñoBallesteros e Tomazella, podemos definir um invariante para a aplicação $f:(V, 0) \rightarrow \mathbb{C}^{d}$ da seguinte forma.

Definição 4.12: Sejam $(V, 0) \subset \mathbb{C}^{n}$ uma ICIS de dimensão complexa d e $f:(V, 0) \rightarrow \mathbb{C}^{d}$ tal que $f^{-1}(0) \cap V$ tem singularidade isolada. Definimos a multiplicidade $\nu(f)$ de $f$ sobre $V$ na origem como sendo,

$$
\nu(f)=\operatorname{grau}(f)+\mu(V, 0)-1 .
$$

Proposição 4.13: Sejam $(V, 0) \subset \mathbb{C}^{n}$ germe de variedade analítica, equidimensional de dimensão $d$ reduzida e $f:(V, 0) \rightarrow \mathbb{C}^{d}$ germe de aplicação analítica, tal que $f^{-1}(0) \cap V$ tem singularidade isolada, então

1. Para d impar temos $\nu(f)=m_{d}(V, 0)-E u_{f, V}(0)$, onde $m_{d}(V, 0)$ denota a d-ésima variedade polar de $V$ na origem.

2. Parad par temos $\nu(f)=m_{d}(V, 0)-E u_{f, V}(0)+2\left(E u_{V}(0)-1\right)$, onde $m_{d}(V, 0)$ denota a d-ésima variedade polar de $V$ na origem. 
Demonstração: Isolando grau de $f$ na relação

$$
E u\left(v^{(d)}, V, \sigma\right)=\operatorname{grau}(f)+E u_{f, V}(\sigma),
$$

e substituindo na Definição 4.12 obtemos $\nu(f)=\left(E u\left(v^{(d)}, V, \sigma\right)-E u_{f, V}(\sigma)\right)+\mu(V, 0)-1$.

Como $(V, 0)$ é uma ICIS $d$-dimensional uma aplicação recursiva da fórmula de Lê-Greuel (ver [46]) temos que

$$
m_{0}(V, 0)-m_{1}(V, 0)+m_{2}(V, 0)+(-1)^{d} m_{d}(V, 0)=1+(-1)^{d} \mu(V, 0),
$$

onde $m_{i}(V, 0)$ é a $i$-ésima variedade polar de $V$ na origem e $\mu(V, 0)$ denota o número de Milnor da ICIS.

Desta fórma, utilizando a Fórmula de Lê e Teissier (Teorema 2.11) obtemos a seguinte relação,

$$
E u_{V}(0)+(-1)^{d} m_{d}(V, 0)=1+(-1)^{d} \mu(V, 0),
$$

de onde segue,

$$
\mu(V, 0)=(-1)^{d} E u_{V}(0)+m_{d}(V, 0)+(-1)^{d+1} .
$$

Usando o Teorema de proporcionalidade (Equação 4.1) e as relações acima obtemos que,

$$
\nu(f)=I\left(v^{(d)}, 0\right) \cdot E u_{V}(0)-E u_{f, V}(\sigma)+(-1)^{d} E u_{V}(0)+m_{d}(V, 0)+\left((-1)^{d+1}-1\right) .
$$

Mas como $v^{d}$ é um $d$-campo radial temos $I\left(v^{(d)}, 0\right)=1$.

De onde obtemos então a relação:

$$
\nu(f)=m_{d}(V, 0)-E u_{f, V}(\sigma)+\left(1+(-1)^{d}\right)\left(E u_{V}(0)-1\right),
$$

de onde segue o resultado.

\subsection{Comentários Finais}

Um problema interessante que pretendemos abordar na continuidade deste trabalho é introduzir a obstrução de Euler de uma aplicação como um invariante para a classificação de germes finitamente determinados $f: \mathbb{C}^{n}, 0 \rightarrow \mathbb{C}^{p}, 0$.

A proposta é utilizar o método introduzido por J. Damon em [15], que relaciona a $\mathcal{A}$-classificação de germes finitamente determinados com a $\mathcal{K}_{V}$-classificação de seções do discriminante $V=\Delta(F)$ de um desdobramento estável $F$ de $f$.

No diagrama abaixo, para cada germe de mergulho $g: \mathbb{C}^{p}, 0 \rightarrow \mathbb{C}^{p} \times \mathbb{C}^{s}, 0$ está associado um germe $f_{g}: \mathbb{C}^{n}, 0 \rightarrow \mathbb{C}^{p}$ obtido como pull-back no diagrama.

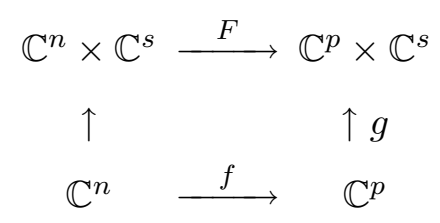


Utilizando resultados de Damon, e o conceito de obstrução de Euler de uma aplicação, podemos associar a cada germe $f_{g}$ um novo invariante, dado pela obstrução de Euler de $E u_{V, \Phi}(\sigma)$, onde $V=\Delta(F), \sigma$ uma célula apropriada, $\Phi: \mathbb{C}^{p} \times \mathbb{C}^{s}, 0 \rightarrow \mathbb{C}^{s}, 0$ é uma submersão, tal que

$$
\Phi^{-1}(0) \cap \Delta(F) \cap U=g\left(\mathbb{C}^{p}\right) \cap \Delta(F) \cap U,
$$

onde $U$ é uma vizinhança suficientemente pequena da origem. Este é o conjunto discriminate de $f_{g}$. 


\section{Referências Bibliográficas}

[1] P. Aluffi, MacPherson's and Fulton's Chern classes of hypersuperfaces, Int. Math. Res. Notices 11 (1994), 455-465.

[2] A. Borel et A. Haefliger, La classe d'homologie fondamental d'un espace analytique, Bull. Soc. Mat. Fr. 89 (1961), 461-513.

[3] A. Borel et F. Hirzebruch, Characteristic classes and homogeneous spaces II. Appendix 1, Amer. J. Math. 81 (1959), 363-371.

[4] J.-P. Brasselet, Classes Características de Variedades Singulares, São Carlos: Notas Didáticas 56 - ICMC - USP - 2002.

[5] J.-P. Brasselet, Local Euler obstruction, old and new, XI Brazilian Topology Meeting (Rio Claro, 1998), World Sci. Publishing, River Edge, NJ, 2000, 140-147.

[6] J.-P. Brasselet, Milnor classes via polar varieties. Singularities in algebraic and analytic geometry (San Antonio, TX, 1999), Contemp. Math., 266, Amer. Math. Soc., Providence, RI, 2000, 181-187.

[7] J.-P. Brasselet, Lê D. T., J. Seade, Euler obstruction and indices of vector fields, Topology, 2000 .

[8] J.P.Brasselet, D. Lehmann, J. Seade, T. Suwa, Milnor Classes of local complete intersections Trans. Am. Math. Soc. 354(2002), no. 4, 1351-1371.

[9] J.-P. Brasselet, D. Massey, A. J. Parameswaran and J. Seade, Euler obstruction and defects of functions on singular varieties, Journal London Math. Soc. (2), 70 (2004).

[10] J.-P. Brasselet et M.-H. Schwartz, Sur les classes de Chern d'un ensemble analytique complexe, Astérisque 82-83 (1981), 93-147.

[11] J.-P. Brasselet, J. Seade and T. Suwa, A proof of the proportionality theorem, Preprint 2005.

[12] T. Bröcker and K. Jänich, Introduction to differential topology, Cambridge University Press, Cambridge-New York, 1982. 
[13] J. W. Bruce and R. M. Roberts Critical points of functions on analytic varieties, Topology Vol. 27, 1988, No 1, 57-90.

[14] S.S. Chern, Characteristic classes of hermitian manifold, Ann. Math. 47 (1946), 85-121.

[15] J. Damon, $\mathcal{A}$-equivalence and the Equivalence of sections of Images and Discriminants, Singularity Theory and Its Applications: Warwick 1989, Vol. 1, Springer Lecture Notes, 1462 (1991), 93-121.

[16] J. Damon, Topological triviality and versality for subgroups of $\mathcal{A}$ and $\mathcal{K}$. II, Sufficient conditions and applications, Nonlinearity 5 (1992), no. 2, 373-412.

[17] J. Damon, The unfolding and determinacy theorems for subgroups of $\mathcal{A}$ and $\mathcal{K}$, Proc. Symp. Pure Math. 40, A.M.S., Providence (1983).

[18] W. Fulton, Intersection Theory, SPRING - VERLAG (1984).

[19] W. Fulton and K. Johnson, Canonical classes on singular varieties, Manuscripta Math.32 (1980), 381-389.

[20] T. Gaffney, Polar multiplicities and equisingularity of map germs, Topology 32 (1993), no. 1, $185-223$.

[21] C. G. Gibson, Singular points of smooth mappings. London: Pitman, 1979.

[22] C. G. Gibson, K. Wirthmuller, A. A. du Plessis and E. J. N. Looijenga, Topological Stability of Smooth Mappings, Lectures Notes In Mathematics 552.

[23] X. Gómez-Mont, J. Seade and A. Verjovsky, The index of a holomorphic flow with an isolated singularity, Math. Ann. 291 (1991), 737-751.

[24] G. Gonzalez-Sprinberg, L'obstruction locale d'Euler et le Théorème de MacPherson, Astérisque 82-83 (1981), 7-32.

[25] V. Goryunov, Functions on space curves, J. London Math. Soc. 61 (2000), 807-822.

[26] M. Goresky a R. MacPherson, Stratified Morse Theory, Springer, Berlins - Heidelberg - New York, 1987.

[27] P. Griffiths, J. Harris Principles of algebraic geometry. New York: John Wiley \& sons, 1978.

[28] A. Grothendieck, Cohomologie locale des faisceaux cohérents et théorèmes de Lefschetz locaux et globaux (SGA 2). Séminaire de Géométrie Algébrique du Bois-Marie, 1962. Advanced Studies in Pure Mathematics, Vol. 2. North-Holland Publishing Co., Amsterdam, 1968.

[29] N. G. Grulha Jr., The Euler Obstruction and Bruce-Roberts' Milnor Number, Préprint 2007.

[30] N. G. Grulha Jr., L'Obstruction d'Euler locale d'une application, a aparecer na revista "Annales de la Faculté des Sciences de Toulouse". 
[31] N. G. Grulha Jr e T. Melo, Sobre Variedades Polares de Superfícies, Preprint 2007.

[32] R. Gunning, H. Rossi Analytic functions of several complex variables. New Jersey: Prentice Hall, 1965.

[33] S.M. Gusein-Zade, I. Luengo, A. Melle-Hernández, On the topology of germs of meromorphic functions and its applications, (Russian) Algebra i Analiz 11 (1999), no. 5, 92-99; translation in St. Petersburg Math. J. 11 (2000), no. 5, 775-780.

[34] R. Hartshorne, Algebraic Geometry, Graduate Texts in Mathematics 52, Springer-Verlag - New York Heidelberg Berlin.

[35] A. Hatcher, Algebraic Topology, Cambridge University Press.

[36] K. Houston, Topology of Differentiable Mappings, Preprint 2006.

[37] T. Izawa, T. Suwa, Multiplicity of functions on singular varieties, Internat. J. Math. 14, 5 (2003), 541-558.

[38] V. H. Jorge Pérez, D. Levcovitz and M. J. Saia, Invariants, equisingularity and Euler obstruction of map germs from $\mathbb{C}^{n}$ to $\mathbb{C}^{n}$, Journal für die Reine und Angewandte Mathematik vol. 587 (2005) 145-167.

[39] S. Kleiman, On the transversality of a general translate, Compositio Math. 28 (1974), 287-297.

[40] Lê D. T., Complex analytic functions with isolated singularities, J. Algebraic Geometry 1 (1992), 83-100.

[41] Lê D. T. , Le concept de singularité isolée de fonction analytique, Adv. Stud. Pure Math., Vol 8, North-Holland, Amsterdam, 1986, 215-227.

[42] Lê D. T., Some remarks on relative monodromy in: P. Holm (Ed.), Real and Complex Singularities, Proceedings of the Nordic Summer School, Oslo 1976, 1977, 397-403.

[43] Lê D. T. and C. P. Ramanujam, The invariance of Milnor's number implies the invariance of the topological type. Amer. J. Math. 98 (1976), no. 1, 67-78.

[44] Lê D. T. et B. Teissier, Cycles évanescents, sections planes et conditions de Whitney. II, Proc. Sympos. Pure Math., vol. 40, part 2, Amer. Math. Soc., Providence, RI, 1983, 65-103.

[45] Lê D. T. et B. Teissier, Variétés polaires Locales et classes de Chern des variétés singulières, Ann. of Math. 114, (1981), 457-491.

[46] E.J.N. Looijenga, Isolated Singular Points on Complete Intersections, London Mathematical Society - Lectures Notes Series 77.

[47] E. L. Lima, A característica de Euler-Poincaré, Matemática Universitária, 1 (1985), 47-62.

[48] E. L. Lima, Variedades diferenciáveis, IMPA, 1973. 
[49] R. D. MacPherson, Chern classes for singular algebraic varieties, Ann. of Math. 100,(1974), 423-432.

[50] J. Mather, Stability of $C^{\infty}$ mappings, III. Finitely determined map-germs. Publications mathématiques de l'L.H.E.S. 35, pg. 127-156, 1968.

[51] J. Mather, Stability of $C^{\infty}$ mappings, IV. Classification of stable germs by R-algebras. Publications mathématiques de l'L.H.E.S. 37, pg. 223-248, 1969.

[52] J. Milnor, Singular points of complex hypersurfaces, Princeton University Press, 1968.

[53] J. Milnor and J. Stasheff, Characteristic classes, Princeton University Press, 1974.

[54] D. Mond and D. van Straten, Milnor number equals Tjurina number for functions on space curves, J. London Math. Soc. 63 (2001), 177-187.

[55] J. J. Nuño-Balesteros and J. N. Tomazella, The Milnor Number of a Function on a Space Curve Germ, a aparecer no Bull. London Math. Soc.

[56] M. A. S. Ruas and J. N. Tomazella, Topological triviality of families of functions on analytic varieties, Nagoya Math. J. 175 (2004), 39-50.

[57] M. A. S. Ruas and J. Tomazella, An infinitesimal criterion for topological triviality of families of sections of analytic varieties, Proc. XII MSJ-IRI Symposium Singularity theory and its applications, 43 (2006) 421-436.

[58] M.-H. Schwartz, Classes caractéristiques définies par une stratification d'une variété analytique complexe, CRAS 260, (1965), 3262-3264 et 3535-3537.

[59] M.-H. Schwartz, Champs radiaux sur une stratification analytique, travaux en cours, 39 (1991), Hermann, Paris.

[60] J. Seade, M. Tibar, and A. Verjovsky, Milnor Numbers and Euler obstruction, Bull. Braz. Math. Soc. (N.S.) 36 (2005), no. 2, 275-283.

[61] J. Seade, M. Tibar and A. Verjovsky, Global Euler obstruction and polar invariants, Math. Ann. 333 (2005), no. 2, 393-403.

[62] M. Sebastiani, Sur la formule de Gonzalez-Verdier, Bull. Braz. Math. Soc. 16 (1985), no. 1, $31-44$.

[63] M. D. Silva, Germes de funçôes sobre variedades analíticas Dissertação de Mestrado - DM Ufscar - 2006.

[64] M. Spivakovsky, Sandwiched singularities and desingularization of surfaces by normalized Nash transformations, Ann. of Math. 131 (1990), 411-491.

[65] N. Steenrod, The topology of fibre bundles., Princeton University Press, 1951. 
[66] T. Suwa, Classes de Chern des intersections completes locales, C. R. Acad. Sci. Paris 124(1996), 67-70.

[67] F. Tari, Singularidades de aplicações diferenciáveis. São Carlos: Notas Didáticas 34 - ICMC USP - 1999.

[68] B. Teissier, Variétés polaires. II. Multiplicités polaires, sections planes et conditions de Whitney, Lecture Notes in Math., 961, Springer, Berlin, 1982.

[69] B. Teissier, Cycles évanescents et résolution simultanée, Séminaire sur les singularités de surfaces, 1976 -77, Springer Lecture Notes 777.

[70] B. Teissier, Cycles évanescents, sections planes et conditions de Whitney, Singularités à Cargèse, Astérisque, 1973.

[71] M. Tibar, Bouquet decomposition of the Milnor fiber, Topology 35 (1996), 1, 227-241.

[72] J. N. Tomazella, Seçôes de Variedades Analíticas, Tese de Doutorado, ICMC - USP - 1999.

[73] J.-L. Verdier, Stratifications de Whitney et théorème de Bertini-Sard, Inventiones Math., 36 (1976), 295-312.

[74] F. Warner, Foundations of differentiable manifolds and Lie groups. New York: Scott, Foresman, 1983.

[75] C. T. C. Wall, Finite Determinacy of smooth map-germs, Bull. London Math. Soc., 13(1981), $481-539$.

[76] H. Whitney, Tangents to an analytic variety, Ann. Math. 81 (3), 496-549 (1965).

[77] O. Zariski and P. Samuel, Commutative Algebra, Volume 1 and 2, Graduate Textbook 28-29, Springer Verlag (1965). 